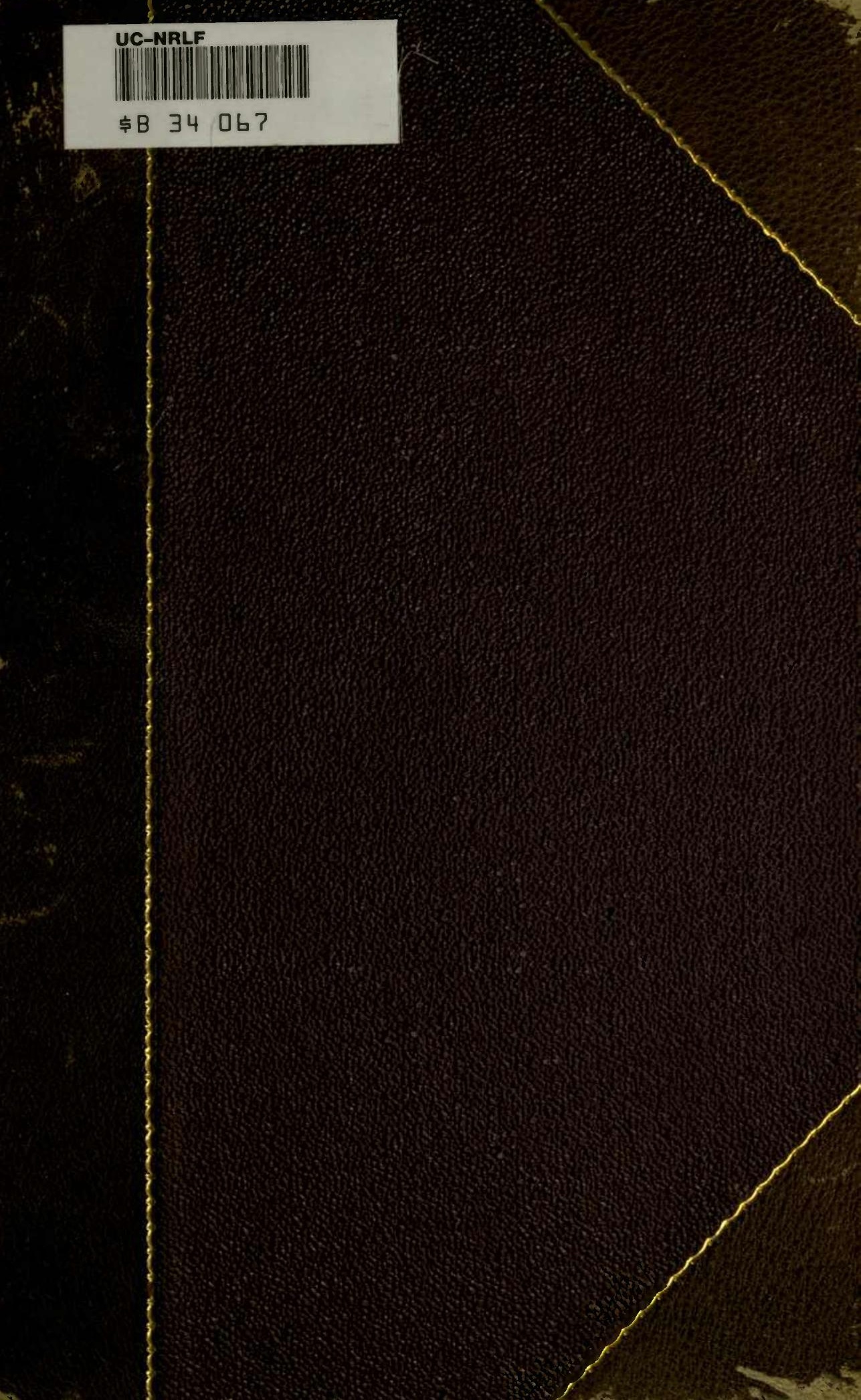




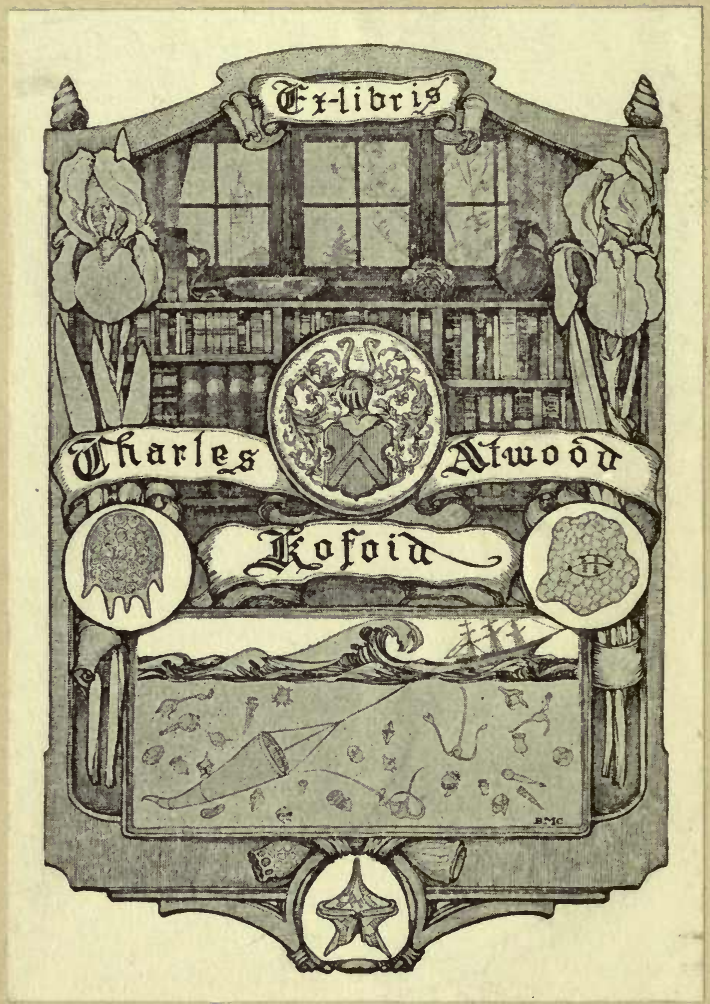




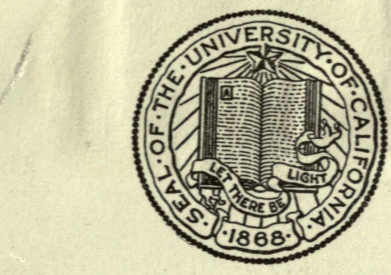

\title{
THE LIBRARY
}

OF

\section{THE UNIVERSITY} OF CALIFORNIA

\author{
PRESENTED BY
}

PROF. CHARLES A. KOFOID AND MRS. PRUDENCE W. KOFOID 





\section{PEDIGREE}

oF

\section{Rysdyk's Hambletonian.}

Sired by Old Abdallah, he by Mambrino, he by imported Messenger. His dam was the Charles Kent Mare, by imported Bellfounder; granddam, old One Eye, by old Hambletonian, and he by imported Messenger, and his dam also by imported Messenger ; and the dam of old One Eye was by imported Messenger. 



\section{870.}

\section{TrotTing STOck,}

${ }^{2 T O N Y-F O R D} L^{T} U_{D}$

ORANGE COUNTY,

New York.

CHARLES BACKMAN. II

\section{NEW YORK;}

Journeymen Printers' Co-operative Association, No 30 Beekman street. 


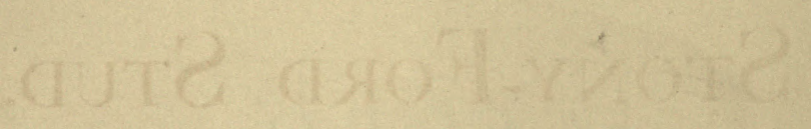




\section{JF 286 $U 5 B 3$}

\section{Stony-Ford Stud.}

\section{STALLIONS.}

ABDALLAH ch. s. foaled June 12, 1863; got by American STAR. Star, Jr., by American Star.

First dam, Curry Abdallah, by Abdallah.

Second dam, by imported Bellfounder.

Third dam, by Royalist.

Fourth dam, by Hardware, by imp. Messenger. American Star, Jr.; got by American Star.

Dam by Friday, he by imported Trustee. 


\section{No. 1.}

ID0L. b. s. foaled June 9, 1864; got by Rysdyk's Hambletonian.

First dam, Hattie Wood, by Harry Clay..

Second dam, Grandmother, by Terror.

Harry Clay; by Cassius M. Clay, Jr. ; he by old Cassius M. Clay.

Dam of Harry Clay, by imported Bellfounder. Cassius M. Clay, Jr.

First dam, by Chancellor, son of Mambrino.

Second dam, by Engineer.

Terror; got by American Eclipse.

First dam, Lady Lightfoot, by Sir Archy.

Second dam, Black Maria, by imported Shark.

Third dam, by imported Clockfast.

Fourth dam, Burwell's Maria, by Regulus.

Fifth dam, Cammilla, by imported Fearnaught. Sixth dam, Calesta, by Forrester.

No. 2.

PIERSON. b. s. foaled May, 1864; got by Rysdyk's Hambletonian.

First dam, by Cassius M. Clay, Jr.

Second dam, by Black Bird.

Cassius M. Clay, Jr., by Cassius M. Clay. Black Bird, by Roebuck, 
No. 3.

MESSENGER b. s. foaled June 3, 1865; got by Rysdyk's DUROC. Hambletonian.

First dam, Satinett, by Abdallah Chief (Roe's).

Second dam, by Whistle Jacket.

Third dam, by the Bertholf Horse.

Fourth dam, by old Duroc.

Abdallah Chief (Roe's), by Abdallah.

First dam, by Phillips, by son of Duroc.

Second dam, by Decatur (thoroughbred), son of Henry.

Whistle Jacket; by Mambrino.

First dam, by American Eclipse.

Bertholf Horse; by imported Messenger.

No. 4.

FITCH. b. s. foaled June, 1865 ; got by Edsell's Clay.

First dam, Lady Edsell, by American Star.

Second dam, by Sea Gull.

Third dam, by Sea Gull.

Edsell's Clay, by Sayre's Harry Clay; dam, by

Post Boy (thoroughbred).

\section{No. 5.}

STONY-FORD. br. s. foaled May, 27, 1866 ; got byRysdyk's Hambletoniau.

First dam, Annie Seely, by Gen'l Gifford.

Second dam, by old Abdallah.

Third dam, by Tom Thumb

Fourth dam, Kent Mare, dam of Rysdyk's Hambletonian. 
No. 6.

WA VERLEY. br. s. foaled October 9,1866 ; got by Rysdyk's Hambletonian.

First dam, Susie Roberts, by N. Y. Black Hawk.

Second dam, the well-known mare of Messenger blood, owned and driven by Captain Roberts, of New York.

N. Y. Black Hawk; by L. I. Black Hawk.

\section{THREE YEAR OLD GELDING.}

ALEXANDER. b. g. foaled April 16, 1867; got by son of Rysdyk's Hambletonian, owned by J. S. Edsell, of Goshen, New York.

First dam, Lady Edsell, by American Star. Second dam, by Sea Gull. Third dam, by Sea Gull.

\section{THREE YEAR OLD COLTS.}

No. 1.

DAUNTLESS. b. c. foaled April 1, 1867; got by Rysdyk's Hambletonian.

First dam, Sallie Feagles, by Smith's Clay.

Second dam, by Hickory.

Third dam, of Messenger Blood. Smith's Clay, by Cassius M. Clay, Jr. 
No. 2.

MENELAUS. br. c. foaled April 14, 1867; got by Rysdyk's Hambletonian.

First dam, Jessie Bull, by L. I. Black Hawk. Second dam of Messenger Blood.

\section{No. 3.}

VICTOR VON b. c. foaled April 19, 1867; got by Rysdyk's BISMARK. Hambletonian.

Full Brother to
Idol. See 'Idol," First dam, Hattie Wood, by Harry Clay. Second, dam Grandmother, by Terror.

\section{No. 4.}

STARTLE. b. c. foaled May 14, 1867; got by Rysdyk's Hambletonian.

First dam, Lizzie Walker, by American Star.

\section{No. 5 .}

AITCHES0N. b. c. foaled June 13, 1867; got by Rysdyk's Hambletonian.

First dam, Lady Van Buren, by Red Bird. Second dam, young Bellfounder, by imported Bellfounder.

\section{No. 6.}

BOLTON. b. c. foaled June 13, 1867; got by Rysdyk's Hambletonian.

First dam, Montgomery Maid, by American Star. 


\section{No. $\%$.}

EXCHANGE. gray c. foaled July 4, 1867; got by Rysdyk's Hambletonian.

Dam, Lady Swazey, by Harris's Hambletonian, by Bishop's Hambletonian, by imported Messenger.

Dam of Harris's Hambletonian was a Messenger mare imported by Israel Munson, of Boston.

No. 8.

BANKER. b. c. foaled June, 1867 ; got by Rysdyk's Hambletonian.

First dam, Lady Banker, by Roe's Abdallah Chief, by Abdallah.

Second dam, by Saltram, by Webber's King Whip.

Third dam, Camptown, by Leopold (thoroughbred), son of Oyle's Oscar.

Abdallah Chief (Roe's), got by Abdallah.

First dam, by Phillips, son of Duroc.

Second dam, by Decatur (thoroughbred), son of Henry.

Saltram: br. h. got by Webber's King Whip; dam, by Seagull, sun of Duroc:

Leopold: ch. h. got by Oyle's Oscar; dam, Katydid, by imp. Expedition. 
No. 9.

KENILWORTH. br. c. foaled Sept. 10, 1867; got by Rysdyk's Hambletonian.

First dam, Susie Roberts, by N. Y. Black Hawk.

Second dam, the well-known mare of Messenger blood, owned and driven by Captain Roberts of New York.

N. Y. Black Hawk; got by L. I. Black Hawk.

\section{TWO YEAR OLD COLTS.}

No. 1.

NETTLE. b. c. foalẹ March 25, 1868; got by Middletown. First dam, Lady Mead, by American Star. Second dam, by Saltram.

Middletown; got by Rysdyk's Hambletonian. First dam, by American Eclipse.

Second dam, by Engineer, the sire of Lady Suffolk.

No. 2.

TWILIGHT. b. c. foaled April 20, 1868; got by Rysdyk's Hambletonian.

First dam, Mary Hulse, by American Star. Second dam, of Messenger blood. 


\section{No. 3.}

ELECTIONEER. b. c. foaled May 2, 1868; got by Rysdyk's Hambletonian.

First dam, Green Mountain Maid, by Harry Clay.

Second dam, by Lexington.

Dam of Harry Clay, by imp. Bellfounder.

\section{No. 4.}

SLIVER HEEL. b. c. foaled May 9, 1868; got by Rysdyk's Hambletonian.

First dam, Rosa Lee, by Harris's Hambletonian, by Bishop's Hambletonian, by imp Messenger.

The dam of Harris's Hambletonian was a Messenger mare, imp. by Israel Munson of Boston.

No. 5.

CUYLER. b. c. foaled May 13, 1868; got by Rysdyk's Hambletonian.

First dam, Grey Rose, by Harris's Hambletonian, by Bishop's Hambletonian, by imp. Messenger.

Dam of Harris's Hambletonian was a Messenger mare, imported by Israel Munson, of Boston. 
No. 6.

TROUBLE. b. c. foaled May 22, 1868; got by Rysdyk's Hambletonian.

First dam, Lady Hopkins, by a son of Hill's Black Hawk.

Second dam, by Harris's Hambletonian.

Dam of Son of Hill's Black Hawk, by Harris's Hambletonian.

No. $\%$.

DIRECTOR. b. c. foaled June 9, 1868; got by Rysdyk's Hambletonian.

First dam, Lady Bellfounder, by Tom Thumb. Second dam, by Crabtree Bellfounder. Third dam, by imported Bellfounder.

No. 8.

WILKINS MICAWBER. b. c. foaled June 24, 1868; got by Rysdyk's Hambletonian.

First dam, Lady Brown, by American Star.

Second dam, by Nigger Lance, he by old Lance (thoroughbred).

\section{No. 9.}

AUGUSTUS. b. c. foaled June 29, 1868; got by Rysdyk's Hambletonian.

First dam, Fanny Fiske, by Plough Boy, he by L. I. Black Hawk.

Second dam, by old Almack, he by Mambrino. 
No. 10.

QUI VIVE. b. c. foaled July 25, 1868; got by Idol.

First dam, Jessie Sayre, by Harry Clay.

Second dam, by Liberty.

Dam of Harry Clay, by imported Bellfounder.

No. 11.

MAMBRINO b. c. foaled 1868; got by Mambrino Pilot.

STAR. First dam, Lady Dexter, by Rysdyk's Hambletonian.

Second dam (Dexter's dam), by American Star.

Mambrino Pilot, got by Mambrino Chief (the sire of Lady Thorn).

Dam by Alexander's Pilot, Jr., the sire of John Morgan.

\section{YEARLING COLTS.}

No. 1.

br. c. foaled April 21, 1869; got by Truesdell's Hambletonian.

First dam, Babbitt's Abdallah, by old Abdallah.

Second dam, by Phillips, son of Duroc.

Third dam, by Decatur (thoroughbred), son of Henry (own sister to Abdallah Chief). 
No. 2.

TRIUMPH. b. c. foaled April 26, 1869 ; got by Idol.

First dam, Rosa Lee, by Harris's Hambletonian, by Bishop's Hambletonian, by imp. Messenger.

The dam of Harris's Hambletonian was a Messenger mare, imported by Israel Munson, of Boston.

\section{No. 3.}

LOADSTONE. b. c. foaled May 7, 1869; got by Idol. First dam, Mary Grimley, by Harry Clay. Second dam, by Terror.

Harry Clay; got by Cassius M. Clay, Jr., he by old Cassius M. Clay.

Dam of Harry Clay by imported Bellfounder. Cassius M. Clay, Jr.; dam by Chancellor, son of Mambrino.

Second dam, by Engineer.

Terror; got by American Eclipse.

First dam, Lady Lightfoot, by Sir Archy. Second dam, Black Maria, by imp. Shark.

Third dam, by imported Clockfast.

Fourth dam, Burwell's Maria, by Regulus. Fifth dam, Cammilla, by imp. Fearnaught. Sixth dam, Calesta, by Forrester.

No. 4.

LUCKNOW. b. c. foaled May 11, 1869 ; got by Idol.

Dam, Grandmother, by Terror. See "Pedigree of Idol " and "Grandmother." 
No. 5.

RIVAL. b. c. foaled May 12, 1869; got by Middletown.

First dam, Mary Hulse, by American Star.

Second dam, of Messenger blood.

See Middletown Pedigree.

No. 6.

LEGACY. b. c. foaled May 15, 1869 ; got by Star, by American Star.

First dam, Lady Ellis, by Alexander's Abdallah (formerly Edsell's Hambletonian).

Dam of Star (Goldsmith's), by Fox Hunter.

No. $\%$.

ARLINGTON. b. c. foaled May 22, 1869; got by Idol.

Dam, Lucy Almack, by young Almack, he by old Almack, he by Mambrino, and he by imp. Messenger.

No. 8.

WO0DSTOCK. b. c. foaled June 1, 1869; got by Idol.

First dam, Sallie Feagles, by Smith's Clay, by

Cassius M. Clay, Jr.

Second dam, a Hickory mare.

Third dam, of Messenger blood.

See Pedigree of Idol. 
No. 9.

BALLO0N B0Y. b. c. foaled June 2, 1869; got by Samson, by Rysdyk's Hambletonian.

First dam, Julia Gavin, by Abdallah Chief (Roe's).

See Abdallah Chief Pedigree.

No. 10.

PICKWICK. br. c. foaled June 9, 1869; got by Idol.

First dam, Lady Brown, by American Star.

Second dam, by Nigger Lance, he by old

Lance (a thoroughbred).

See Pedigree of Idol.

No. 11.

TWINKLE. g. c. foaled June 15, 1869; got by Idol.

First dam, Belle of Richmond, by Rysdyk's Hambletonian.

Second dam, old Belle of Richmond, by Grey

Norman.

\section{No. 12.}

RAVENSW00D. br. c. foaled June 25, 1869; got by Messenger Duroc.

First dam, Virgo, by Abdallah Chief.

Second dam, by Whistle Jacket.

Third dam, by the Bertholf horse.

Fourth dam, by old Duroc.

See Pedigree of Messenger Duroc. 
No. 13.

TELLER. b. c. foaled June 26, 1869 ; got by Idol.

Dam, Grey Rose, by Harris's Hambletonian, by Bishop's Hambletonian, by imported Messenger.

Dam of Harris's Hambletonian was a Messenger mare, imported by Israel Munson, of Boston.

No. 14.

RED GAUNTLET. ch. c. foaled June 28, 1869 ; got by Idol. First dam, Annie Seeley, by Gen'l Gifford.

Second dam, by Old Abdallah.

Third dam, by Tom Thumb.

Fourth dam, Kent mare (dam of Rysdyk's Hambletonian).

\section{No. 15.}

PROSPER0. blk. c. foaled July 12, 1869; got by Messenger Duroc.

First dam, Green Mountain Maid, by Harry Clay.

Second dam, by Lexington.

\section{No. 16.}

GARDNER. ch. c. foaled August 19, 1869; got by Messenger Duroc.

Frst dam, Lady Overton, by old Abdallah. Second dam, by Mount Holly, Mount Holly by Mambrino, 
No. 17.

ch. c. foaled September 1,1869; got by Idol. First dam, Lady Farr, by old Abdallah.

\section{No. 18.}

ch. c. foaled September 9, 1869; got by Messenger Duroc.

First dam, Hattie Wood, by Harry Clay.

Second dam, Grandmother, by Terror.

Harry Clay; got by Cassius M. Clay, Jr., he by old Cassius M. Clay.

Dam of Harry Clay, by imp. Bellfounder.

Cassius M. Clay, Jr.

First dam, by Chancellor, son of Mambrino.

Second dam, by Engineer.

Terror; got by American Eclipse.

First dam, Lady Lightfoot, by Sir Archy.

Second dam, Black Maria, by imp. Shark.

Third dam, by imported Clockfast.

Fourth dam, Barwell's Maria, by Regulus.

Fifth dam, Cammilla, by imported Fearnaught.

Sixth dam, Calesta, by Forrester.

WILMARTH. ch. c. foaled September 9, 1869; got by Idol.

Dam, Nellie Wilmarth, by Kemble Jackson, 
No. 20.

ch. c. foaled April 6, 1869; got by Pahallas, he by Rysdyk's Hambletonian.

First dam, Lady Rockafellow, by American Star.

Second dam, by Bay Richmond,

Third dam, by Gilbert Oliver's Jackson, by son of Messenger.

Pahallas; got by Rysdyk's Hambletonian.

First dam, Nicky, he by Mambrino Messenger, he by Mambrino.

Second dam, by Post Boy, he by Sir Henry, he by Sir Archy.

\section{BROOD MARES.}

No. 1.

ROAN MARIE. $\mathrm{rn}, \mathrm{m}, 16$ hands, foaled 1853 ; got by a horse from Canada.

Dam, by Abdallah,

No. 2.

NELLIE. ch. m. $15^{\frac{1}{2}}$ hands, foaled 1854 ; got by young Star, owned by Thomas Bingham, of Newburgh, N. Y. First dam, by Wild Air. 
No. 3.

GREY ROSE. gr. m. $15^{\frac{1}{2}}$ hands, got by Harris's Hambletonian, he by Bishop's Hambletonian, by imported Messenger.

Dam of Harris's Hambletonian was a Messenger mare, imported by Israel Munson, of Boston.

No. 4.

KATE SMITH. b. m. 15-1 hands; got by old Abdallah.

No. 5.

BELLE. ch. m. 15.3 hands, foaled 1861; got by Post's Hambletonian, by Rysdyk's Hambletonian.

First dam, by Ethiopian Prince, by Wild Air. Second dam, from Ohio.

Post's Hambletonian; got by Rysdyk's Hambletonian.

First dam, said to be by Bellfounder.

Second dam said to be of Messenger blood.

\section{No. 6.}

ALICE VAN DUZER. blk. m. 15 hands, foaled May, 1852; got by American Star.

First dam, by Messenger Duroc.

Second dam, by Paymaster. 
No. $\%$.

HATTIE WOOD. b. m. 15.1 hands, foaled 1860 ; got by Harry Clay.

First dam, Grandmother, by Terror.

Harry Clay; got by Cassius M. Clay, Jr., he by old Cassius M. Clay.

Dam of Harry Clay, by imported Bellfounder. Cassius M. Clay, Jr.

First dam, by Chancellor, son of Mambrino.

Second dam, by Engineer.

Terror; got by American Eclipse.

First dam, Lady Lightfoot, by Sir Archy.

Second dam, Black Maria, by imported Shark. Third dam, by imported Clockfast, Fourth dam, Burwell's Maria, by Regulus. Fifth dam, Cammilla, by imported Fearnaught. Sixth dam, Caselta, by Forrester.

No. 8.

KATE SEELY. b. m. 15.3 hands, foaled 1849 ; got by Walden's Messenger, by Wild Air, he by son of imp. Messenger.

First dam, by Mambrino, by imp. Messenger.

No. 9.

LADY BROWN. br. m. 15 hands, foaled 1861; got by American Star.

First dam, by Nigger Lance, by old Lance, a thoroughbred, 


\section{No. 10.}

LADY MEAD. b. m. 16 hands, foaled 1856 ; got by American Star.

Dam, by Saltram.

\section{No. 11.}

FANNY FERN. b. m. 15.1 hands, foaled April, 1852; got by American Star.

First dam, by Sea Gull, by Duroc.

Second dam, by Sea Gull, by Duroc.

\section{No. 12.}

LIZZIE WALKER. ch. m. 15 hands; got by American Star.

\section{No. 13.}

LADY BELLFOUNDER. ch. m. 15.2 hands, foaled 1856 ;

"Known as the , got by Tom Thumb.

First dam, by Crabtree's Bellfounder.

Second dam, by imported Bellfounder.

No. 14.

ROSA LEE. gr. m. 15 hands ; got by Harris's Hambletonian, by Bishop's Hambletonian, by imp: Messenger.

Dam of Harris's Hambletonian was a Messenger mare, imported by Israel Munson, of Boston, 
No. 15.

GRANDMOTHER. ch. m. 15.1 hands ; got by Terrror, by American Eclipse.

Terror's first dam, Lady Lightfoot, by Sir Archy. Second dam, Black Maria, by imported Shark. Third dam, by imported Clockfast.

Fourth dam, Burwell's Maria, by Regulus.

Fifth dam, Cammilla, by imported Fearnaught. Sixth dam, Calesto, by Forrester.

No. 16.

JESSIE SAYRE. br. m. $15.1 \frac{1}{2}$ hands, foaled 1861 ; got by Harry Clay, he by Cassius M. Clay, Jr.

Dam, by Liberty.

Dam of Harry Clay, by imported Bellfounder.

No. 17.

SALLIE FEAGLES. br. m. 15.2 $\frac{1}{2}$ hands, foaled 1854 ; got by Smith's Clay, by Cassius M. Clay, Jr.

First dam, by Hickory.

Second dam, of Messenger blood.

No. 18.

LUCY ALMACK. b. m. 15.2 hands, foaled 1857; got by young Almack, by old Almack, by Mambrino, by Imported Messenger.

No. 19.

MAGGIE JONES. ch. m. 15.1 hands, foaled 1856 ; got by American Star.

Dam, by Mambrino Chief. 
No. 20.

ANNIE SEELY. b. m. $15.1_{\frac{1}{2}}$ hands, foaled 1858; got by Gen'l Clifford.

First dam, by old Abdallah.

Second dam, by Tom Thumb.

Third dam, Kent mare, dam of Rysdyk's Hambletonian.

Gen'l Gifford; got by Gifford Morgan, he by Green Mountain Morgan.

Second dam, by Tom Thumb, was the dam of Green's Bashaw, sire of Faucett's Bashaw and Sam Kirkwood.

No. 21.

LADY DARLING. ch. m. 15 hands, foaled 1860 ; got by American Star.

Dam by Nigger Lance, he by old Lance.

\section{No. 22.}

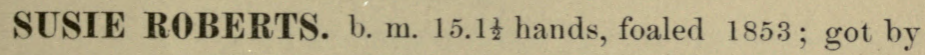
N. Y. Black Hawk, he by L. I. Black Hawk.

Dam, the well-known mare of Messenger blood, owned and driven by Captain Roberts, of New York.

\section{No. 23.}

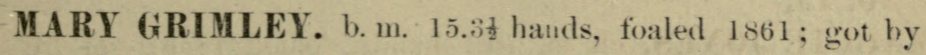

Harry Clay.

Dam by Terror. 


\section{No. 24.}

MISS WANSOR. b. m. 16.1 hands, foaled 1860 ; got by Sir Archy, by Green Mountain Morgan.

First dam, by Abdallah.

Second dam, by Westchester by L. I. Black Hawk.

No. 25.

SEELY ABIDLLAH. b. m. 15.3 hands, foaled 1847; got by old Abdallah.

First dam, by Tom Thumb.

Second dam, Kent mare (dam of Rysdyk's Hambletonian).

No. 26.

LADY HAWIKINS. b. m. 15.2 hands, foaled 1850 ; got by old Abdallah.

Dam, Emily, by Commodore, he by Mambrino.

No. $2 \%$.

BELLE OF RICHMOND. gr m. 15.1 hands, foaled 1861 ; got by Rysdyk's Hambletonian.

Dam, old Belle of Richmond, by Grey Norman.

No. 28.

LADY PACKER. gr. in. 15.2 hands, foaled 1860 ; got by Witherell's Messenger, by Winthrop's Messenger, by Bush Messenger, by imported Messenger. 
กัo. $2 \hat{9}$.

AGNES BOYLE. b. m. 15.3 hands, foaled 1856 ; got by Full sister to Post's Rysdyk's Hambletonian.
Hambletonian.

First dam said to be by Bellfounder.

Second dam, said to be of Messenger blood.

No. 30.

MISTRESS KENYON. ch. m. 15.2 hands; got by Abdallah Chief (Roe's), by old Abdallah.

Dam, by Sir Henry.

\section{No. 31.}

MILDRED. b. m. 15 hands, foaled 1858; got by Rysdyk's Hambletonian.

Dam, Linda (the old trotting mare).

\section{No. 32.}

GREEN MOUNTAIN MAID. br. m. 15 hands, foaled 1862 ; got by Harry Clay.

Dam, by Lexington.

Dam of Harry Clay, by imported Bellfounder.

No. 33.

KITTY MILLS. b. m. 15.1 hands; got by American Star. Dam, by Wild Air.

No. 34.

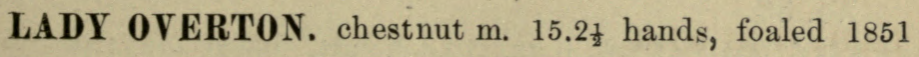
got by old Abdallah.

Dam, by Mt. Holly, by old Mambrino. 
No. 35.

SATINETT. blk. m. 15.2 hands, foaled 1855 ; got by Abdallah Chief (Roe's).

First dam, by Whistle Jacket.

Second dam, by the Bertholf Horse.

Third dam, by Old Duroc.

Abdallah Chief (Roe's); by Abdallah.

First dam, by Phillips, by son of Duroc.

Second dam, by Decatur (thoroughbred), son of Henry.

Whistle Jacket, by Mambrino.

Dam, by American Eclipse.

Bertholf Horse, by imp. Messenger.

No. 36.

NELLIE WILMARTH. br. m. 15 hands; got by Kemble Jackson.

No. $3 \%$.

SPY. br. m. 15.2 hands; got by Botts's Revenue.

Dam, by Red Eye by Boston.

No. 38.

FANNIE FISKE. b. m. 15.2 hands, foaled 1853 ; got by Plough Boy, by L. I. Black Hawk.

Dam, by old Almack, by Mambrino.

\section{No. 39.}

LADY HOPKINS. b. m. 15.3 hands, foaled 1856 ; got by son of Hill's Black Hawk.

Dam, by Harris's Hambletonian. 
No. 40.

JENNIE LEWIS. br. m. 15.2 hands, foaled 1850 ; got by Black Bashaw.

No. 41.

MONTGOMERY MAID. blk. m. 15.3 hands, foaled 1849; got by American Star.

\section{No. 42.}

LADY BRYANT. br. m. 15.2 hands, foaled 1862; got by Brignoli, by old Mambrino Chief.

Dam, by Cracker, by Boston.

Bred by David Bryant, Lexington, Ky.

\section{No. 43.}

VIRG0. blk. m. 15.1 hands, foaled 1856 ; got by Abdallah

Chief (Roe's).

First dam, by Whistle Jacket.

Second dam, by the Bertholf horse.

Third dam, by old Duroc.

Abdallah Chief (Roe's); by Abdallah.

First dam by Phillips, by son of Duroc.

Second dam, by Decatur (thoroughbred), son of Henry.

Whistle Jacket; by Mambrino.

First dam, by American Eclipse.

Bertholf Horse; by imp. Messenger. 
No. 44.

LADY ELLIS. b. m. 15.3 hands, foaled 1860 ; got by Alexander's Abdallah, formerly Edsell's Hambletonian.

No. 45.

MARY HULSE. b. m. 15 bands, foaled 1860; got by American Star.

Dam of Messenger blood.

No. 46.

FANNIE STAR. b. m. 15.2 hands, foaled 1860 ; got by American Star.

Dam by old Abdallah.

No. $4 \%$.

TRUSTUS WEBB. b. m. 15.2 hands, foaled 1860 ; got by American Star.

Dam, by Trustee.

No. 48.

CURRY ABDALLAH. chestnut m. 16 hands, foaled 1847; got by old Abdallah.

First dam, by imported Bellfounder,

Second dam, by Royalist.

Third dam, by Hardware, by imp. Messenger. 
No. 49.

SHARPLESS ABDALLAH. br. m. 15.1 hands, got by old Abdallah.

Dam, by Hunter (sire of Flora Temple and the D'Lancy mare called Mist).

No. 50.

LADY PIERSON. b. m. 16 hands, foaled, 1853; got by Cassius M. Clay, Jr.

First dam, by imported Diamond.

Second dam, a running mare, said to be thoroughbred.

No. 51.

LADY NODINE. br. m. 15.1 hands, foaled 1863 ; got by Rysdyk's Hambletonian.

Dam, Montgomery Maid, by American Star.

\section{No. 52.}

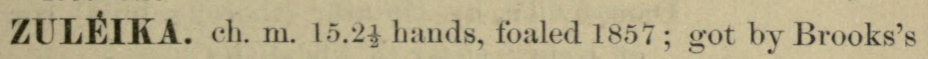
Black Hawk, by L. I Black Hawk.

Dam, by Mambrino Chief.

\section{No. 53.}

LADY CLAY. blk, m. 15.1 hands, foaled 1863; got by Cardinal.

Dam, by imported Bellfounder. 
No. 54.

MAT'IIE b. m. 15.2 hands, foaled 1866; got by Rysdyk's Hambletonian.

First dam, Lady Earle, by Chauncey Green.

Second dam, by Judson's Hambletonian.

Third dam, by Harris's Hambletonian.

Fourth dam, by Judson's Hambletonian.

Fifth dam, by Harris's Hambletonian.

Chauncey Green, by Hale's Morgan.

Dam by Judson's Hambletonian.

No. 55.

BONNIE LASSIE. b. m. $15.1 \frac{1}{2}$ hands, foaled 1865 ; got by Rysdyk's Hambletonian.

Dam, by Fox Hunter.

No. 56.

BABBITT ABDALLAH. b. m. 15 hands, foaled 1850 ; got by old Abdallah.

First dam, by Phillips, son of Duroc.

Second dam, by Decatur (thoroughbred), son of Henry, sister to Abdallah Chief.

No. $5 \%$.

AM ANDA. b. m. 15.1 hands, foaled 1866 ; got by Rysdyk's Hambletonian.

First

Second dam, by One-Eyed Hunter. 
No. 58.

MOLLIE GREY. gr. m. 15.2 hands; got by young Columbus. Dam by Harris's Hambletonian.

\section{No. 59.}

COQUETTE. b. m. 15.2 hands, foaled 1863; got by Wild Irishman, by Harry Clay.

Dam said to be by American Star.

Dam of Wild Irishman, by American Star.

\section{No. 60.}

LADY DEXTER. b. m. 15 hands, foaled 1861; got by (Full sister of Rysdyk's Hambletonian.
Dexter.)

Dam, by American Star.

No. 61.

MATTIE BULL. got by Cassius M. Clay, Jr.

First dam, by Ohio Eclipse.

Second dam, by Post Boy.

No. 62.

EMMA MILLS. ch. m. 15.1 hands, foaled 1853 ; got by

(Dam of

"Independent.")

Dam, by Alexander W., by Alexander (thoroughbred, from Virginia).

No. 63.

LADY FARR. b. m. 15 hands; got by old Abdallah. 


\section{No. 64 .}

BONITA. b. m. $15_{\frac{1}{2}}$ hands, foaled May, 1863 ; got by George Miller.

Dam, Brown Pacer.

\section{No. 65.}

LADY BANKER. b. m. 15.2 hands, foaled Spring 1855; got by Roe's Abdallah Chief,

First dam, by Saltram, by Webber's King Whip.

Second dam, Camptown, by Leopold, thoroughbred, son of Oyles' Oscar

Abdaliah Chief (Roe's); got by Abdallah.

First dam, by Phillips, son of Duroc.

Second dam, by Decatur (thoroughbred), son of Henry.

Saltram; br. h. got by Webber's King Whip.

Dam by Sea Gull, son of Duroc.

Leopold; ch. h. got by Oyles' Oscar.

Dam, Katydid, by imported Expedition.

No. 66.

FLORA FEAGLES. b. m. 15.3 hands, foaled 1866 ; got by Rysdyk's Hambletonian.

Dam Sallie Feagles, by Smith's Clay.

No. $6 \%$.

FANNY MAPES. b. m. 15.1 hands; got by Alexander's Ab. dallah, known as Edsell's Hambletonian. 
No. 68.

LEDGER GIRL. br. m. 15.1 hands, foaled 1863; got by Rysdyk's Hambletoniau.

Dam, by son of Barny Henry.

No. 69.

GLENCOE MAID. br. m. foaled 1855; got by Glencoe.

No. 70.

KATE. blk. m. 14.3 hands, foaled 1852 ; got by Belaire, the Bruno's Dam. sire of Red Bird.

Dam, a Pacing mare, of Comic blood.

No. 71.

STELLA. b. m. $14 \frac{1}{2}$ hands.

No. 72.

ELLA HOWLAND. ch. m. $15 \frac{1}{\frac{1}{2}}$ hands; got by young Jupiter.

First dam, by American Star.

Second dam, by Sea Gull.

Third dam, by Sea Gull.

No. 73.

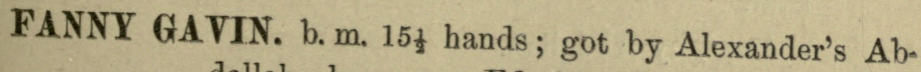
dallah; known as Edsell's Hambletonian. 
No. 74.

LADY FALLIS. b. m. 15.3 hands, foaled 1859 ; got by American Star.

- Dam, by L. I. Black Hawk.

No. 75.

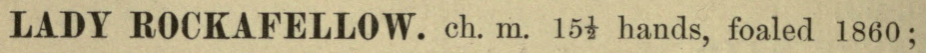
got by American Star.

First dam, by Bay Richmond.

Second dam, by Gilbert Oliver's Jackson, by son of Messenger.

No. 76.

SMITH'S STAR. ch. m. 15.1 hands, foaled 1860 ; got by American Star.

Dam, by L. I. Black Hawk.

No. $7 \%$.

WELLS' STAR. blk. m. 15 hands, foaled 1866; got by American Star.

Dam, by Bertrand, he by Sir Solomon, he by Sir Archy.

No. 78.

LADY ABDALLAH. ch. m. 15.3 hands; got by American Star.

Dam, by Abdallah. 


\section{SIX YEAR OLD MARE.}

EMMA. b. m. foaled May, 1864; got by son of Hill's Black Hawk.

Dam, Grey Rose, by Harris's Hambletonian.

Dam of son of Hill's Black Hawk, by Harris's Hambletonian. 


\section{FIVE YEAR OLD MARES.}

No. 1.

FAIRY. b. m. foaled 1865; got by Rysilyk's Hambletonian. First dam, Emma Mills, by American Star. Second dam, by Alexander W., by Alexander (thoroughbred), from Virginia.

\section{No. 2.}

GAZELLE. b. m. foaled 1865; got by Rysdyk's Hambletonian.

First dam, Hattie Wood, by Harry Clay.

Second dam, Grandmother, by Terror.

Harry Clay; by Cassius M. Clay, Jr.; he by old Cassius M. Clay.

Dam of Harry Clay, by imported Bellfounder. Cassius M. Clay, Jr.

First dam, by Chancellor, son of Mambrino.

Second dam, by Engineer.

Terror; got by American Eclipse.

First dam, Lady Lightfoot, by Sir Archy.

Second dam, Black Maria, by imported Shark.

Third dam, by imported Clockfast.

Fourth dam, Burwell's Maria, by Regulus.

Fifth dam, Cammilla, by imported Fearnaught.

Sixth dam, Calesta, by Forrester.

\section{No. 3.}

NELLIE TEMPLE. b. m. foaled 1865; got by Rysdyk's Hambletonian.

Dam, Jennie Lewis, by blk. Bashaw. 


\section{FOUR YEAR OLD MARES.}

No. 1.

HELEN. br. m. foaled 1866 ; got by Rysdyk's Hambletonian. Dam, by Rockwell's Rattler, son of Biggott's Rattler.

No. 2.

EFFIE DEANS. br. m. foaled 1866; got by Rysdyk's Hambletonian.

First dam, by young Fiddler.

Second dam, by Abdaliah.

Young. Fiddler's dam, by Ohio Eclipse.

\section{No. 3.}

LADY MOSCOW. br.m. foaled Aug. 5, 1866 ; got by Rysdyk's Hambletonian.

First dam, Roan Marie.

Second dam, by Abdallah.

No. 4.

HOPE. b. m. foaled October 22, 1866; got by Rysdyk's Hambletonian.

First dam, Fanny. Fern, by American Star.

Second dam, by Sea Gull.

Third dam, by Sea Gull. 
No. 5.

b. m. foaled May, 1866 ; got by Rysdyk's Hamletonian.

First dam, Lady Banker, by Abdallah Chief (Roe's).

Second dam, by Saltram, by Webber's King Whip.

Third dam, Camptown, by Leopold (thoroughbred), son of Oyles' Oscar.

Abdallah Chief (Roe's), got by Abdallah.

First dam, by Phillips, by Duroc.

Second dam, by Decatur (thoroughbred), son of Henry.

Saltram; br. h. got by Webber's King Whip.

Dam, by Sea Gull, son of Duroc.

Leopold; ch. h. got by Oyles' Oscar.

Dam, Katydid, by imported Messenger.

No. 6.

LADY HILL. b. m. foaled October 22, 1866 ; got by Rysdyk's Hambletonian.

Dam, by Harry Clay.

Dam of Harry Clay by imported Bellfounder. 


\section{THREE YEAR OLD FILLIES.}

No. 1.

YOUNG DUCHESS. br. f. foaled 1867 ; got by Iron Duke, he by Rysdyk's Hambletonian.

First dam, by Cardinal.

Second dam, by One-Eyed Hunter.

No. 2.

RACOLA. br. f. foaled April 22, 1867; got by Rysdyk's Hambletonian.

Dam, Brown Pacer.

No. 3.

ROSETTA. b. f. foaled April 22, 1867 ; got by Rysdyk's Hambletonian.

First dam, Grey Rose, by Harris's Hambletonian, by Bishop's Hambletonian, by imported Messenger.

Dam of Harris's Hambletonian was a Messenger mare, imported by Israel Munson, of Boston.

No. 4.

CALYPSO. b. f. foaled July 4, 1867 ; got by Rysdyk's Hambletonian.

Dam, Kate Smith, by old Abdallah. 


\section{No. 5 .}

JUNO. b. f. foaled April 24, 1867; got by Rysdyk's Hambletonian.

First dam, Satinett, by Abdallah Chief (Roe's).

Second dam, by Whistle Jacket.

Third dam, by the Bertholf Horse.

Fourth dam, by Old Duroc.

Abdallah Chief (Roe's); by Abdallah.

First dam, by Phillips, by Duroc.

Second dam, by imp. Messenger.

Whistle Jacket, by Mambrino.

Dam, by American Eclipse.

Bertholf Horse, by imp. Messenger.

No. 6.

BELLONA. b. f. foaled April 26, 1867; got by Rysdyk's Hambletonian.

First dam, Lady Bellfounder, by Tom Thumb.

Second dam, by Crabtree's Bellfounder. Third dam, by imported Bellfounder.

No. $\%$.

MAY. b. f foaled April 30, 1867; got by Middletown. First dam, Kitty Mills, by American Star. Second dam, by Wild Air. 
No. 8.

DONNA. b. f. foaled May 9, 1867; got by Fitzgerald's Hambletonian.

First dam, Annie Seely, by Gen'l Gifford.

Second dam, by Abdallah.

Third dam, by Tom Thumb.

Fourth dam, Kent Mare (dam of Rysdyk's Hambletonian).

No. 9.

STORM. b. f. foaled May 21, 1867; got by Middletown. First dam, Green Mountain Maid, by Harry Clay.

Second dam, by Lexington.

No. 10.

JOSEPHINE. b. f. foaled August 13, 1867; got by Rysdyk's Hambletonian.

Dam, Lucy Almack, by young Almack, by Abdallah, by Mambrino, by imported Messenger.

\section{No. 11.}

GAY SPANKER. br. f. foaled July 9, 1867; got by Rysdyk's Hambletonian.

First dam, Roan Marie.

Second dam, by Abdallah. 
No. 12.

BESS. b. f. foaled August 16, 1867 ; got by Rysdyk's Hambletonian.

First dam, Jessie Sayre; by Harry Clay. Second dam, by Liberty.

No. 13.

COSETTE. b. f. foaled October 30,1867 ; got by Rysdyk's Hambletonian.

First dam, Kate Seely by Walden Messenger, by Wild Air.

Second dam, by Mambrino, by imported Messenger.

No. 14.

ZENOBIA. br. f. foaled September 18, 1867 ; got by Rysdyk's Hambletonian.

First dam, Belle, by Post's Hambletonian, by Rysdyk's Hambletonian.

Second dam, by Ethiopian Prince, by Wild Air. Third dam, from Ohio, 


\section{TWO YEAR OLD FILLIES.}

No. 1.

VIOLET. b. f. foaled March 16, 1868; got by Rysdyk's Hambletonian.

First dam, Sallie Feagles, by Smith's Clay, he by Cassius M. Clay. Second dam, by Hickory. Third dam, of Messenger blood.

No. 2.

ZERLINA. b. f. foaled April 19, 1868; got by Rysdyk's Hambletonian.

First dam, Annie Seeley, by Gen'l Gifford. Second dam, by Old Abdallah.

Third dam, by Tom Thumb.

Fourth dam, Kent mare (dam of Rysdyk's Hambletonian). 


\section{No. 3.}

BLOSS0M. b. f. foaled May 1, 1868; got by Rysdyk's Ham bletonian.

First dam, Hattie Wood, by Harry Clay.

Seeond dam, Grandmother, by Terror.

Harry Clay; got by Cassius M. Clay, Jr., he by old Cassius M. Clay.

Cassius M. Clay, Jr.:

First dam, by Chancellor, son of Mambrino.

Second dam, by Engineer.

Terror; got by American Eclipse.

First dam, Lady Lightfoot, by Sir Archy.

Second dam, Black Maria, by imp. Shark.

Third dam, by imported Clockfast.

Fourth dam, Burwell's Maria, by Regulus.

Fifth dam, Cammilla, by imported Fearnaught.

Sixth dam, Calesta, by Forrester.

No. 4.

QUEEN OF MAY. b. f. foaled May 1, 1868; got by Rysdyk's Hambletonian.

First dam, Lady Overton, by Abdallah.

Second dam, by Mt. Holly, by Mambrino.

No. 5.

CARRIE. b. f. foaled May 17, 1868, got by Woburn.

Dam, Jennie Lewis, by Black Bashaw.

No. 6.

HERMINE. b. f. foaled May 28, 1868; got by Sackett's Hambletonian, he by Rysdyk's Hambletonian.

Dam, Julia Gavin, by Abdallah Chief (Roe's). 
No. 7.

STARBEAM. b. f. foaled June 12, 1868; got by Rysdyk's Hambletonian.

Dam, Montgomery Maid, by American Star.

No. 8.

MARGUERITE. b. f. foaled June 18,1868 ; got by Rysdyk's Hambletonian.

Dam, Kate Smith, by Abdallah.

\section{No. 9.}

UNDINE. b. f. foaled June 26, 1868; got by Rysdyk's Hambletonian.

First dam, Roan Maria.

Second dam by Abdallah.

\section{No. 10.}

PATTI. br. f. foaled August 12, 1868; got by Idol.

First dam Susie Roberts, by N. Y. Black Hawk, he by L. I. Black Hawk.

Second dam, the well-known Messenger mare, owned and driven by Captain Roberts, of New York.

\section{No. 11.}

ILMA. br. f. foaled August 22, 1868; got by Idol. First dam, Belle, by Post's Hambletonian. Second dam, Ethiopian Prince, by Wild Air. Third dam, from Ohio. 


\section{No. 12.}

MINEOLA. b. f. foaled May, 1868; got by Rysdyk's Hambletonian.

First dam, Lady Banker, by Abdallah Chief (Roe's).

Second dam, by Saltram, by Webber's King Whip.

Third dam, Camptown, by Leopold (thoroughbred).

Abdallah Chief (Roe's); got by Abdallah.

First dam by Phillips, son of Duroc.

Second dam, by Decatur (thoroughbred), son of Henry.

Saltram; br. h. got by Webber's King Whip.

Dam, by Sea Gull, son of Duroc.

Leopold; ch. h. got by Oyles' Oscar.

Dam, Katydid, by imported Expedition. 


\section{YEARLING FILLIES.}

\section{No. 1.}

b. f. foaled April 25, 1869; got by Chosrose.

First dam, Lady Fallis, by American Star.

Second dam, by. L. I. Black Hawk.

\section{No. 2.}

BELLE R0E. ch. f. foaled May 2, 1869; got by Idol. First dam, Satinett, by Abdallah Chief (Roe's).

Second dam, by Whistle Jacket.

Third dam, by the Bertholf Horse.

Fourth dam, by old Duroc.

Abdallah Chief (Roe's), by Abdallah.

First dam, by Phillips, by Duroc.

Second dam, by Decatur, son of Henry.

Whistle Jacket, by Mambrino.

Dam, by American Eclipse.

Bertholf Horse, by imported Messenger.

No. 3.

b. f. foaled May 6, 1869; got by Tom Wonder.

First dam, Wells's Star, by American Star.

Second dam, by Bertrand, by Sir Solomon, by Sir Archy. 
No. 4.

ADA DUR0C. b. f. foaled May 15, 1869; got by Messenger Duroc.

Dam, Montgomery Maid, by American Star.

No. 5.

ZYPHER. b. f. foaled May 15, 1869; got by Idol.

First dam, Miss Wansor, by Sir Archy, by Green Mountain Morgan.

Second dam, by Abdallah.

Third dam, by Westchester, by L. I. Black Hawk.

No. 6.

IDA WEBSTER. b. f. foaled May 24, 1869; got by Rysdyk, he by Rysdyk's Hambletonian.

First dam, Sharpless Abdallah, by Abdallah. Second dam, by Hunter (sire of Flora Temple and De Lancy mare, called Mist).

No. $\%$.

EVELINA. b. f. foaled June 9, 1869; got by Idol.

First dam, Mistress Kenyon, by Abdallah Chief (Roe's).

Second dam, by Sir Henry. 
No. 8.

FLIRT. ch. f. foaled June 10, 1869; got by Messenger Duroc. First dam, Nellie, by Young Star horse, owned by Thos. Bingham, of Newburgh.

Second dam, by Wild Air.

No. 9.

SUNBEAM. b. f. foaled June 20, 1869 ; got by Idol.

Dam, Lady Packer, got by Witherell Messenger, by Winthrop Messenger, by imported Messenger.

No. 10.

PERICHOLE. b. f. foaled July 3 , 1869 ; got by Idol.

First dam, Kate Seely, by Walden Messenger, by Wild Air.

Second dam, by Mambrino, by imported Messenger.

No. 11.

MIGNONNETTE. ch. f. foaled July 18, 1869; got by Idol.

First dam, Maggie Jones, by American Star.

Second dam, by Mambrino Chief.

\section{No. 12.}

MADELINE. b. f. foaled August 6, 1869; got by Abdallah Star.

First dam, Fannie Fiske, by Plough Boy, by L. I. Black Hawk.

Second dam, by old Almack by Mambrino. 


\section{No. 13.}

BOULOTTI. b. f. foaled August 17, 1869; got by Idol. First dam, Lady Mead, by American Star. Second dam, by Saltram.

\section{No. 14.}

BELLA WILFER. b. fo foaled August 19, 1869; got by Idol.

First dam, Belle, by Post's Hambletonian, he by Rysdyk's Hambletonian.

Second dam, by Ethiopian Prince, by Wild Air. Third dam, from Ohio.

\section{No. 15.}

b. f. foaled October 23, 1869; got by Idol.

First dam, Bonita, by Geo. Miller.

Second dam, Brown Pacer.

No. 16.

b. f. foaled 1869 ; got by Bellfounder.

First dam, Kate (Bruno's dam), by Belaire, the sire of Red Bird.

Second dam, Pacing mare, of Comic Blood.

\section{NOTICE.}

I am now ready to offer a portion of my Stock at Private Sale during the Year. 


\section{PEDIGREE}

OF

\section{Rysdyk's Hambletonian.}

Sired by Old Abdallah, he by Mambrino, he by imported Messenger. His dam was the Charles Kent Mare, by imported Bellfounder; granddam, old One Eye, by old Hambletonian, and he by imported Messenger, and his dam also by imported Messenger; and the dam of old One Eye was by imported Messenger. 



\section{87 I.}

\section{Trotting Stock,}

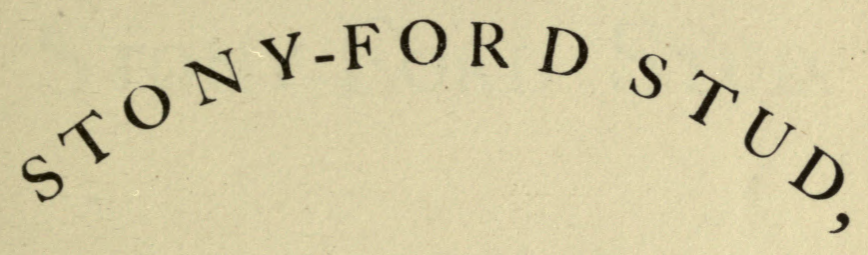

\section{ORANGE COUNTY,}

New York.

CHARLES BACKMAN.

NEW YORK :

Henry Spear, Printer and Stationer, 82 W all Street. 



\section{Stony-Ford Stud.}

\section{STALLIONS.}

ABDALLAH ch. s. 15.3 hands, small star, white off hind STAR. ankle, foaled June 12, 1863; got by American Star, Jr., by American Star.

First dam, Curry Abdallah, by Abdallah.

Second dam, by imported liellfounder.

Third dam, by Royalist.

Fourth dam, by Hardware, by imp. Messenger.

American Star, Jr. ; got by American Star.

Dam by Friday, he by imported Trustre. 


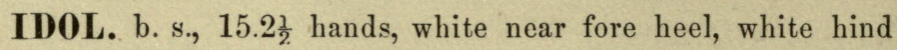
ankles, foaled June 9, 1864; got by Rysdyk's Hambletonian.

First dam, Hattie Wood, by Harry Clay.

Second dam, Grandmotber, by Terror.

Harry Clay; by Cassius M. Clay, Jr., he by old Cassius M. Clay.

Dam of Harry Clay, by imported Bellfounder. Cassius M. Clay, Jr.

First dam, by Chancellor, son of Mambrino.

Second dam, by Engineer.

Terror; got by American Eclipse.

First dam. Lady Lightfont, by Sir Archy.

Second dam, Black Maria, by imported Shark.

Third dam, by imported Clockfast.

Fourth dam, Burwell's Maria, by Regulus.

Fifth dan, Cammilla, by imported Fearnaught.

Sixth dam, Calesta, by Forrester.

PIERSON. b. s. 16 hands, white hind heels, foaled May, 1864 ; got oy Rysdyk's IJambletonian.

First dam, by Cassius M. Clay, Jr.

Second dam, by Black Bird.

Cassius M. Clay, Jr., by Cassius M. Clay. Black Bird, by Roebuck. 
MESSENGER b. s., 16 hands, white hind ankles, foaled DU ROC. June 3, 1865; got by Rysdyk's Hambletonian. First dam, Satinet, by Abdallah Chief (Roe's).

Second dam, by Whistle Jacket.

Third dam by the Bertholf Horse.

Fourth dam, by old Duroc.

Abdallah Chief (Roe's), by Abdallah.

First dam, by Phillips, by son of Duroc.

Second dam, by Decatur (thoroughbred), son of Henry.

Whistle Jacket, by Mambrino.

First dam, by American Eelipse.

Bertholf Horse ; by imported Messenger.

STONY-FORD. br. s., 15.1 hands, little white on near hind heel. foaled May 27, 1866; got by Rysdyk's Hambletonian.

First dam, Annie Seely, by Gen'l Gifford.

Second dam, by old Abdallah.

Third dam, by Tom Thumb.

Fourth dam, Kent Mare, dam of Rysdyk's Hambletonian.

WA VERLEY. br. s., 15.3 hands, star, white hind ankles, white off fore heel, foaled October 9, 1866; got by Rysdyk's Hambletonian.

First dam Susie Roberts, by N. Y. Black Hawk.

Second dam, the well-known mare of Messenger blood, owned and driven by Captain Roberts, of New York,

N. Y. Black Hawk, by L. I. Black Hawk. 


\section{FOUR YEAR OLD COLTS.}

No. 1.

DAUNTLESS. b. c., small star, black points, foaled April 1, 1867 ; got by Rysdyk's Hambletonian.

First dam, Sallie Feagles, by Smith's Clay.

Second dam, by Hickory

Third dam, of Messenger Blood.

Smith's Clay, by Cassius M. Clay, Jr.

No. 2.

MENELAUS. br. c. star, white hind ankles dotted with black, foaled April 14, 1867 ; got by Rysdyk's Hambletonian.

First dam, Jessie Bull, by L. I. Black Hawk. Second dam of Messenger blood.

No. 3.

VICTOR VON b. c., white hind heels, foaled April 19, 1867 ;

BISMARCK. got by Rysdyk's Hambletonian.

Full Brother to
Idol. See 'Idol,' First dam, Hattie Wood, by Harry Clay.

Iage 6.
Iol.

No. 4.

AITCHES0N. b. c., star, white near hind ankle, foaled June 13, 1867 ; got by Rysdyk's Hambletonian

First dam, Lady Van Buren, by Red Bird.

Second dam, young Bellfounder, by imported Bellfounder. 
No. 5.

BOLTON. b. c.. white off hind heel, foaled June 13, 1867; got by Rysdyk's Hambletonian.

First dam, Montgomery Maid, by American Star.

No. 6.

EXCHANGE. g. c., star and snip, white hind ankles, foaled July 4, 1867; got by Rysdyk's Hambletonian.

Dam, Lady Swazey, by Harris's Hambletonian, by Bishop's Hambletonian, by imported Messenger.

Dam of Harris's Hambletonian was a Messenger mare, imported by Israel Munson, of Boston.

\section{No. $\%$.}

KENIL W0RTH. br. e, black points, foaled Sept. 10, 1867 got by Rysdyk's Hambletonian.

First dam, Susie Roberts, by N. Y. Black Hawk.

Second dam, the well-known mare of Messenger blood. owned and driven by Captain Roberts, of New York.

N. Y. Black Hawk; got by L. I. Black Hawk.

\section{THREE YEAR OLD COLTS.}

\section{No. 1.}

TWILIGHT. b. c. white hind heels, foaled April 20, 1868 ; got by Rysdyk's Hambletonian.

First dam, Mary Hulse, by American Star.

Second dam, of Messenger blood. 
No. 2.

ELECTIONEER. b. ca, white hind heels, foaled May 2, 1868, got by Rysdyk's Hambletonian.

First dam, Green Mountain Maid, by Harry Clay.

Second dam, by Lexington.

Dam of Harry Clay, by imp. Bellfounder.

No. 3.

CUYLER. b. c., star, near white hind ankle, foaled May 13, 1868 ; got by Rysdyk's Hambletonian.

First dam, Grey Rose, by Harris's Hambletonian, by Bishop's Hambletonian, by imp. Messenger.

Dam of Harris's Hambletonian was a Messenger mare, imported by Israel Munson, of Boston.

No. 4.

TROUBLE. b. c , white hind ankles, foaled May 22, 1868 ; got by Rysdyk's Hambletonian.

First dam, Lady Hopkins, by a son of Hill's Black Hawk.

Second dam, by Harris's Hambletonian.

Dam of Son of Hill's Black Hawk, by Harris's Hambletonian.

\section{No. 5.}

DIRECTOR. b. c., small star, white hind ankles, foaled June 9, 1868 ; got by Rysdyk's Hambletonian. First dam, Lady Bellfounder, by Tom Thumb. Second dam, by Crabtree Bellfounder. Third dam, by imported Bellfounder. 
No. 6.

MAMBRINO b. c., white hind heels, white near foot, foaled STAR. 1868; got by Mambrino Pilot.

First dam, Lady Dexter, by Rysdyk's Hambletonian.

Second dam (Dexter's dam), by American Star.

Mambrino Pilot, got by Mambrino Chief (the sire of Lady Thorn).

Dam by Alexander's Pilot, Jr., the sire of John Morgan.

\section{THREE YEAR OLD GELDING.}

\section{No. 1.}

SILVER HEEL. b c. white near hind heel, foaled May 9, 1868 ; got by Rysdyk's Hambletonian

First dam, Rosa Lee, by Harris's Hambletonian, by Bishop's Hambletonian, by imp. Messenger.

The dam of Harris's Hambletonian was a Messenger mare, imp. by Israel Munson, of Boston.

\section{TWO YEAR OLD GELDINGS.}

No. 1.

LUCKNOW. b. g., has star and snip, white near hind heel, white off fore heel, foaled May 11, 1869 ; got by Idol.

Dam, Grandmother, by Terror. See "Pedigree of Idol " and "Grandmother." 


\section{No. 2.}

PICK WICK. br. g., star and snip, white hind ankles, little white on near fore heel, foaled June 9, 1869 ; got by Idol.

First dam, Lady Brown, by American Star.

Second dam, by Nigger Lance, he by old I ance [a thoroughbred].

See Pedigree of Idol.

\section{No. 3.}

PROSPERO. blk. g., white hind heels, foaled July 12, 1869 ; got by Messenger Duroc.

First dam, Green Mountain Maid, by Harry Clay.

Second dam, by Lexington.

\section{TWO YEAR OLD COLTS.}

No. 1.

TRIUMPH. b. c., white hind heels, foaled April 26, 1869; got by Idol.

First dam, Rosa Lee, by Harris's Hambletonian, by lishop's Hambletonian, by imp. Messenger.

The dam of Harris's Hambletonian was a Messenger mare, imported by Israel Munson, of Boston.

No. 2.

GARDNER. ch. c., star, white hind heels, foaled August 19, 1869; got by Messenger Duroc.

First dam, Lady Overton, by old Abdallah.

Second dam, by Mount Holly, Mount Holly by Mambrino. 
No. 3

GEORGE ch. e., star and snip, white hind heels, white GARDINER. near fore heel, foaled September 9, 1869; got by Messenger Duroc.

First dam Hattie Wood, by Harry Clay.

Second dam, Grandmother, by Terror.

Harry Clay; got by Cassius M. Clay, Jr., he by old Cassius M. Clay.

Dam of Harry Clay, by imp. Bellfounder.

Cassius M. Clay, Jr.

First dam, by Chancellor, son of Mambrino

Second dam, by Engineer.

Terror; got by American Eclipse.

First dam, Lady Lightfoot, by Sir Archy.

Second dam, Black Maria, by imp. Shark.

Third dam, by imported Clockfast.

Fourth dam, Burwell's Maria, by Regulus.

Fifth dam, Cammilla, by imported Fearnaught.

Sixth dam, Calesta, by Forrester.

\section{YEARLING COLTS.}

No. 1.

b. c., white hind ankles, foaled March 8th, 1870 , got by Pierson.

First dam, Lady Hopkins, by son of Hill's Black Hawk.

Second dam, by Harris's Hambletonian.

\section{No. 2.}

b. c., black points, foaled April 18th, 1860; got by Messenger Duroc.

First dam, Trustus Webb.

Second dam by Trustee. 
No. 3.

b. c., star, white near hind ankles, foaled April 21st, 1870 ; got by Mills Hambletonian.

First dam, Lady Fallis by American Star.

Second dam, by I. I. Black Hawk.

No. 4.

b. c., strip in face, white off fore ankle, white hind ankles, foaled April 24th, 1870; got by Pierson First dam, Zuleika, by Brooks' Black Hawk, by L. I. Black Hawk.

Second dam, by Mambrino Chief.

\section{No. 5.}

Elander H. b. c., white off heels, foaled April 26th, 1870; got Sherman by Idol.

First dam, Mattie, by Rysdyk's Hambletonian. Second dam, Lady Earle, by Chauncy Green. Third dam, by Judson's Hambletonian.

Fourth dam, by Harris' Hambletonian.

Fifth dam, by Judson's Hambletonian.

Sixth dam, Harris' Hambletonian.

Chauncy Green, by Hale's Morgan, dam by Judson's Hambletonian. 


\section{No: 6.}

b. c., star white hind ankles, foaled May 3d, 1870, got by Comet.

First dam, Lady Banker, by Abdallah Chief (Roe's).

Second dam by Saltram, by Kentucky Whip. Third dam, Camptown, by Leopold (thoroughbred), son of Ogles Oscar.

Abdallah Chief (Roe's), got by Abdallah.

First dam, by Phillips, son of Duroc.

Second dam, by Decatur (thoroughbred), son of Henry; Saltram, br. h., got by Webber's Kentucky Whip, dam by Sea Gull, son of Duroc.

Leopold, ch. h., got by Ogles' Oscar, dam Katy Did, by imported Expedition.

No. $\%$.

b. c, white hind ankles, foaled May 5th, 1870, got by Pierson.

First dam, Alice Van Duzer, by American Star.

Second dam by old Messenger Duroc.

Third dam by Paymaster.

No. 8 .

b. c., star, white near hind heel, foaled May 18th, 1870, got by Conductor.

First dam, Emma Hoyt, by American Star.

Second dam by Ohio Eclipse.

Third dam, a mare of Messenger blood. 


\section{No. 9.}

ch. c, strip in face, white hind legs, foaled May 21st, 1870, got by Messenger Duroc.

First dam, Fannie Star, by American Star.

Second dam by old Abdallah.

No. 10.

ch. c. star, white hind ankles, foaled May 21st, 1870, got by Messenger Duroc.

First dam, Grey Rose, by Harris' Hambletonian.

Dam of Harris' Hambletonian was a Messen. ger mare, imported by Israel Munson, of Boston.

No. 11.

BELMON'T. b. c., white hind heels, foaled May 22d, 1870, got by Rysdyk's Hambletonian.

First dam, Miss Wansor, by Sir Archy.

Second dam by Abdallah.

Third dam by Westchester.

\section{No. 12.}

b. c., snip on nose, white fore heels, white hind ankles, foaled June 2d, 1870, got by Messenger Duroc.

First dam, Lady Cardinal, by Cardinal.

Second dam, by imported Bellfounder. 


\section{No. 13.}

blk. c, white near fore heel, white hind heels, foaled June 5th, 1870; got by Messenger Duroc.

First dam Montgomery Maid, by American Star.

\section{No. 14.}

MAJOR MORTON. b. c, star, white hind heels, foaled June 20th, 1870; got by Rysdyk's Hambletonian. First dam, Maggie Jones, by American Star.

Second dam, by Mambrino Chief.

\section{No. 15.}

ch. c., star, white hind heels, foaled June 25th, 1870 ; got by Messenger Duroc.

First dam, Lady Nodine, by Rysdyk's Hambletonian.

Second dam, Montgomery Maid, by American Star.

\section{No. 16.}

b c., star, white off hind heel, white near hind ankle, white near fore leg; foaled July $3 d, 1870$, got by Rysdyk's Hambletonian.

First dam, Fannie Fiske, by Plough Boy, by L. I. Black Hawk.

Second dam, by old Almack, by Mambrino. 
No. 17.

b. c, white hind heels, foaled July 6th, 1870 ; got by Pierson.

First dam Roan Marie, by horse from Canada. Second dam by Abdallah.

No. 18.

b. c., star, white hind ankles, foaled July 14th, 1870 , got by Messenger Duroc.

First dam Virgo, by Abdallah Chief (Roe's).

Second dam by Whistle Jacket.

Third dam by the Bertholf horse.

Fourth dam by old Duroc.

Abdallah Chief (Roe's), by Abdallah.

First dam by Phillips, by son of Duroc.

Second dam by Decatur (thoroughbred), son of Henry.

Whistle Jacket, by Mambrino.

First dam by American Eclipse.

Bertholf Horse, by imported Messenger.

\section{No. 19.}

b. c., star, white hind ankles, foaled Aug. 12th, 1870, got by Pierson, dam, Kate Smith, by old Abdallah.

\section{BROOD MARES.}

No. 1.

ROAN MARIE. rn. m. 16 hands, foaled 1853; got by a horse' from Canada.

Dam, by Abdallah. 
No. 2.

GREY ROSE. gr. m. $15 \frac{1}{2}$ hands, got by Harris' Hambletonian, he by Bishop's Hambletonian, by imported Messenger.

Dam of Harris' Hambletonian was a Messenger mare, imported by Israel Munson, of Boston.

No. 3.

BELLE. ch. m. star and snip, little white on hind heels, 15.3 hands, foaled 1861; got by Post's Hambletonian, by Rysdyk's Hambletonian.

First dam, by Ethiopian Prince, by Wild Air.

Second dam, from Ohio.

Post's Hambletonian; got by Rysdyk's Hambletonian.

First dam, said to be by Bellfounder.

Second dam said to be of Messenger blood.

\section{No. 4.}

ALICE VAN DUZER. blk. m. 15 hands, strip in face, white hind ankles, white near fore heel, foaled May, 1852; got by American Star.

First dam, by Messenger Duroc.

Second dam, by Paymaster.

\section{No. 5 .}

KATE SEELY. b. m. 15.3 hands, white near hind heel, (Dam of stallion foaled 1849; got by Walden's Messenger, by Charles Backman,
owned by Wild Air, he by son of imp. Messenger. w.Minibury,Mass.) First dam, by Mambrino, by imp. Messenger. 
No. 6.

LADY RROWN. br m 15 hands, star and snip, white off (Dam of Gauntlet hind heel, foaled 1861; got by American Star. wilkins Micawber.) First dam, by Nigger Lance, by old Lance, a thoroughbred.

No. $\%$.

LADY MEAD. b. m. 16 hands, star and snip, white near (Dam of Nettle.) hind ankle, foaled 1856 ; got by American Star. Dam, by Saltram.

No. 8.

LIZZIE WALKER. ch. m. 15 hands; star and snip, white (Dam of Startle). hind legs, white off fore heel, yot by American Star.

First dam, by old Hickory ; Hickory by Whip ; Whip by Seymour's Wild Air; Wild Air by Fearnaught.

No. 9.

LADY BELLFOUNDER. ch. m. 15.2 hands, strip in face, "Wnown as the , white near hind leg up to hock, white off hind (Dam of Bellona.) ankle, foaled 1856 ; got by Tom Thumb. First dam, by Crabtree's Bellfounder. Second dam, by imported Bellfounder.

No. 10.

ROSA LEE. gr. m. 15 hands; got by Harris' Hambletonian, by Bishop's Hambletonian, by imp. Messenger. Dam of Harris' Hambletonian was a Messenger mare, imported by Israel Munson, of Bopston. 
No. 11.

GRANDMOTHER. ch. m. 15.1 hands; got by Terror, by American Eclipse.

Terror's first dam, Lady Lightfoot, by Sir A rehy. Decond dam, Black Maria, by imported Shark. Third dam, by imported Clockfast.

Fourth dam, Burwell's Maria, by Regulus.

Fifth dam, Cammilla, by imported Fearnaught. Sixth dam, Calesto, by Forrester.

\section{No. 12.}

SALLIE FEAGLES. br. m. $15.2 \frac{1}{2}$ hands, black points, foaled 1854; got by Smith's Clay, by Cassius M. Clay, Jr.

First dam, by Hickory, by Mambrino, by imp Messenger.

Second dam, of Messenger blood.

\section{No. 13.}

LUCY ALMACK. b. m. 15.2 hands. star, white off hind ankle, white near fore heel, foaled 1857; got by young Almack, by old Almack, by Mambrino, by Imported Messenger.

\section{No. 14.}

MAGGIE JONES. ch. m. 15.1 hands, star, little white on near hind heel, fraled 1856; got by American Star.

Dam, by Mambrino Chief. 


\section{No. 15.}

ANNIE SEELY. b. m. $15.1 \frac{1}{2}$ hands, black points, foaled 1858; got by Gen'l Clifford.

First dam, by old Abdallah.

Second dam, by Tom Thumb.

Third dam, Kent mare, dam of Rysdyk's Hambletonian.

Gen'l Gifford; got by Gifford Morgan, he by Green Mountain Morgan.

Second dam, by Tom Thumb, was the dam of Green's Bashaw, sire of Faucett's Bashaw and Sam Kirkwood.

No. 16.

LADY DURLAND. ch. m. 15 hands, star and snip, foaled 1860 ; got by American Star.

Dam by Nigger Lance, he by old Lance.

No. $1 \%$.

SUSIE ROBER'TS. b. m. $15.1 \frac{1}{2}$ hands, black points, foaled 1853; got by N. Y. Black Hawk, he by L. I. Black Hawk.

Dam, the well-known mare of Messenger blood, owned and driven by Captain Roberts, of New York.

No. 18.

MISS WANSOR. b. m. 16.1 hands, black points, foaled 1860 ; got by Sir Archy.

First dam, by Abdallah.

Second dam, by Westchester. 
No. 19.

LADY HAWKINS. b. m. 15.2 hands, white off hind heel, foaled 1850; got by old Abdallah.

Dam, Emily, by Commodore, he by Mambrino.

No. 20.

BELLE OF RICHMOND. gr. m. 15.1 hands, white near hind ankle, foaled 1861; got by Rysdyk's Hambletonian.

Dam, old Belle of Richmond, by Grey Norman.

\section{No. 21.}

LADY PACKER. gr. m. 15.2 hands, black points, foaled 1860 ; got by Witherell's Messenger, by Winthrop's Messenger, by Bush Messenger, by imported Messenger.

\section{No. 22.}

AGNES BOYLE. b. m. 15.3 hanàs, strip in face, white near Full sister to
Post's Hambletonian. tonian.

First dam said to be by Bellfounder.

Second dam, said to be of Messenger blood.

\section{No. 23.}

MISTRESS HENYON. ch. m. 15.2 hands; star and strip, white hind legs, white near fore heel; got by Abdallah Chief (Roe's), by old Abdallah.

Dam, by Sir Henry. 
No. 24.

MILDRED. b. m. 15 hands, star, white off hind ankle, foaled 1858; got by Rysdyk's Hambletonisn.

Dam, Linda (the old trotting mare).

No. 25.

GREEN MOUNTAIN MAID. br. m. 15 hands, star, white hind ankles, foaled 1862 ; got by Harry Clay.

Dam, by Lexington.

Dam of Harry Clay, by imported Bellfounder.

No. 26.

KITTY MILLS. b. m. 15.1 hands; snip in face, black points; got by American Star.

Dam, by Wild Air.

No. $2 \%$.

LADY OVERTON. ch. m. $15.2 \frac{1}{2}$ hands, strip in face, white off hind ankle, foaled 1851; got by old Abdallah.

Dam, by Mt. Holly, by old Mambrino.

No. 28.

NELLIE WILMARTH. br. m. 15 hands; star, white hind heels; got by Kemble Jackson. 
No. 29.

SPY. br. m. 15.2 hands; strip on face, spot on nose, white off hind heel; got by Bott's Revenue.

Dam by Red Eye, by Boston

No. 30.

FANNIE FISKE. b. m. 15.2 hands, white near hind leg, white fore heel, foaled 1853; got by Plough Boy, by L. I Black Hawk.

Dam, by old Almack, by Mambrino

\section{No. 31.}

LADY HOPKINS. b. m. 15.3 hands, white off hind heel, foaled 1856 ; got by son of Hill's Black Hawk.

Dam, by Harris' Hambletonian.

\section{No. 32.}

MONTGOMERY MAID. blk. m. 15.3 hands, star, white over off nostril, white hind ankles, foaled 1849 ; got by American Star.

\section{No. 33.}

VIRG0. blk. m. 15.1 hands, white near hind heel, foaled 1856; got by Abdallah Chief (Roe's).

First dam, by Whistle Jacket.

Second dam, by the Bertholf horse.

Third dam, by old Duroc.

Abdallah Chief (Roe's); by Abdallah.

First dam, by Philips, by son of Duroc.

Second dam, by Decatur (thoroughbred), son of Henry.

Whistle Jacket; by Mambrino.

First dam, by American Eclipse.

Bertholf Horse ; by imp. Messenger. 


\section{No. 34.}

LADY ELLIS. b. m. 15.3 hands, white near hind ankle, foaled 1860 ; got by Alexander's Abdallah, formerly E'dsell's Hambletonian.

No. 35.

MARY HULSE. b. m. 15 hands, white hind ankles, foaled 1860 ; got by American Star.

Dam of Messenger blood.

No. 36.

FANNIE STAR. b. m. 15.2 hands, black points, foaled 1860 ; got by American Star.

Dam, by old Abdallah.

No. 37.

TRUSTUS WEBB. b. m. 15.2 hands, black points, foaled 1860 ; got by American Star.

Dam, by Trustee.

No. 38.

CURRY ABDALLAH. ch. m. 16 hands, star and snip,

white hind heels, foaled 1847; got by old Abdallah.

First dam, by imported Bellfounder.

Second dam, by Royalist.

Third dam, by Hardware, by imp. Messenger. 
No. 39.

SHARPLESS ABDALLAH. br. m. 15.1 hands, white off hind heel, got by old Abdallah.

Dam, by Hunter (sire of Flora Temple and the De Lancy mare called Mist).

No. 40.

LADY PIERSON. b. m. 16 hands, star and snip, foaled 1853; got by Cassius M. Clay, Jr.

First dam, by imported Diamond.

Second dam, a running mare, said to be thoroughbred.

No. 41.

LADY NODINE. br. m. 15.1 hands, white hind heels, foaled 1863; got by Rysdyk's Hambletonian. Dam, Montgomery Maid, by American Star.

No. 42.

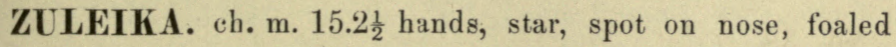
1857; got by Brooks' Black Hawk, by L. I. Black Hawk.

Dam, by Mambrino Chief. 
No. 43.

LADY CARDINAL. blk. m. 15.1 hands, star and snip, white legs, foaled 1863 ; got by Cardinal.

Dam, by imported Bellfounder.

No. 44.

MATTIE. b. m. 15.2 hands, black points, foaled 1866; got (Dam of Elander H. Sherman.)

by Rysdyk's Hambletonian.

First dam, Lady Earle, by Chauncey Green.

Second dam, by Judson's Hambletonian.

Third dam, by Harris' Hambletonian.

Fourth dam, by Judson's Hambletonian.

Fifth dam, by Harris' Hambletonian.

Chauncey Green, by Hale's Morgan.

Dam, by Judson's Hambletorian.

No. 45.

BONNIE LASSIE. b. m., star and snip, white near hind heel, $15.1 \frac{1}{2}$ hands, foaled 1865; got by Rysdyk's Hambletonian.

Dam, by Fox Hunter.

No. 46.

BABBITT ABDALLAH. b. m. 15 hands, black points, foaled 1850; got by old Abdallah.

Pirst dam, by Phillips, son of Duroc.

Second dam, by Decatur (thoroughbred), son of Henry, sister to Abdallah Chief. 


\section{No. 47.}

LADY DEXTER. b. m. 15 hands, strip in face, white legs, (Fall sister of
Dexter.) foaled 1861; got by Rysdyk's Hambletonian. Dam, by American Star.

\section{No. 48.}

MATTIE BULL. b. m. 15.3 hands, star and snip, white hind heels, white near fore ankle, white off fore heel, foaled May 20th, 1853; got by Cassius M. Clay, Jr.

First dam, by Ohio Eclipse.

Second dam, by Post Boy.

No. 49.

EMMA MILLS. ch. m. 15.1 hands, strip in face, white near "Independent.") hind heel, foaled 1853; got by American Star. Dam, by Alexander W., by Alexander (thoroughbred, from Virginia.)

No. 50 .

LEDGER GIRL. br. m. 15.1 hands, sinall star, black points, foaled 1863 ; got by Rysdyk's Hambletonian.

Dam, by son of Barney Henry. 
No. 51.

GLENCOE MAID. ch. m. 15.1 hands, strip in face, near white hind ankle, foaled 1855 ; got by Glencoe.

No. 52.

KATE. blk. m. 143 hands, strip in face, white off hind ankle, Bruno's Dam. white near hind heel, white near fore ankle, foaled 1852; got by Bellaire, the sire of Red liird.

Dam, a pacing mare, of Comic blood.

\section{No. 53.}

STELLA. b. m. $14 \frac{1}{2}$ hands, strip in face, white near fore heel, white near hind ankle.

\section{No. 54.}

LADY FALLIS. b m. 15.3 hands, strip in face, foaled (Dam of Socrates.) 1859; got by American Star.

Dam, by L. I. Black Hawk.

No. 55.

LADY ROCKAFELLOW. ch. m. $15 \frac{1}{2}$ hands, strip in face, $\underset{\substack{\text { (Dam of the } \\ \text { Kerner Colt.) }}}{\text { white hind legs, foaled 1860; got by American }}$ Star.

First dam, by Bay Richmond.

Second dam, by Gilbert Oliver's Jackson, by son of Messenger. 
No. 56.

WELLS' STAR. br. m. 15.1 hands, star and snip, white off hind heel, foaled 1866; got by American Star.

Dam, by Bertrand, he by Sir Solomon, he by Sir Archy.

No. $5 \%$.

HATTIE HOGAN. br. m. 15.3 hands, star and snip, white hind heels, foaled 1867, got by Harry Clay.

First dam, by American Star.

Second dam, by Hickory.

No. 58.

NELLIE SAYRES. br. m. 15.1 hands, star, white off hind heel, foaled got by American Star.

Dam, by Hickory.

No. 59.

EMMA HOY'T. ch. m. $15 \frac{1}{2}$ hands, star and snip, white near hịnd heel, foaled 1859, got by American Star.

First dam by Ohio Eclipse.

Second dam, a mare of Messenger blood.

No. 60.

WAVERLEY HOWLAND. b. m. $15 \frac{1}{2}$ hands, star and snip, white near hind heel, foaled got by American Star.

First dam by Black Bird.

Black Bird by American Eclipse. 
No. 61.

ADELE. b. m. $15 \frac{1}{2}$ hands, star and snip, white hind ankles, white near fore heel, foaled 1867 , got by Rysdyk's Hambletonian.

Dam, Lady Franklin, (owned by David Bonner, Esq.)

No. 62.

LADY IRWIN. b. m. 15.3 hands, spot on nose, white near hind ankle, white near fore heel, foaled got by Rysdyk's Hambletonian.

First dam, by Abdallah Chief (Roe's).

No. 63.

JANE MURRAY. g. m. 15.1 hands, foaled 1853, got by (Dam of Lottery.)

No. 64.

FAIRY. b. m. 15.2 hands, white hind ankles, foaled 1865 ; got by Rysdyk's Hambletonian.

First dam, Emma Mills, by American Star.

Second dam, by Alexander W., by Alexander (thoroughbred), from Virginia. 


\section{No. 65.}

KITTIE HARKER. b. m. 15.2 hands, strip in face, white off hind heel, white spot on off fore heel, foaled, got by Ethan Allen.

First dam, Martha, by Abdallah.

Second dam, by young Bellfounder.

Tbird dam, by Corn Cracker.

Fourth dam, by Hiskory.

Abdallah, by Mambrino.

Dam, Smazonia, by imported Messenger.

Young Bellfounder, by imported Bellfounder.

Dam by Wild Air.

Corn Cracker, b. h. foaled 1838, by imported Tranby.

Dam, Mary Haxwell, by Moses. 


\section{SIX YEAR OLD MARE.}

NELLIE TEMPLE. b. m. 15.3 hands, white hind ankles, foaled 1865; got by Rysdyk's Hambletonian. Dam, Jennie Lewis, by blk Bashaw. 


\section{FIVE YEAR OLD MARES.}

No. 1.

HELEN, br. m. 15.1 hands, star, white hind heels, foaled 1866/, got by Rysdyk's Hambletonian.

Dam, by Rockwell's Ratiler, son of Biggott's Rattler.

No. 2.

EFEIE DEANS. b m. 15.3 hands, star, white hind ankles, foaled 1866 ; got by Rysdyk's Hambletonian.

First dam, by young Fiddler.

Second dam, by Abdallah.

Young Fiddler's dam, by Ohio Eclipse.

No. 3.

LADY MOSCOW. r. m., black points, foaled Aug. 5, 1866 ; got by Rysdyk's H:mbletonian.

First dam, Roan Marie.

Second dam, by Abdallah.

No. 4.

HOPE. b. m., star and snip, white off hind heel, foaled October 22, 1866; got by Rysdyk's Hambletonian.

First dam, Fanny Fern, by American Star.

Second dam, by Sea Gull.

Third dam, by Sea Gull. 
No. 5 .

AMA NDA. b. m 15.1 hands, star and snip, little white on off hind heel, foaled 1866; got by Rysdyk's Hambletonian.

First dam, Lady Cardinal, by Cardinal.

Second dam, by One-Eyed Hunter.

No. 6.

FLORA FEAGLES. b. m. 16 hands, star, black points, foaled 1866 ; got by Rysdyk's Hambletonian. Dam, Sallie Feagles, by Smith's Clay.

No. $\%$.

LADY HILL. b. m. 14 hands, star and snip, little white on hind heels, foaled October 22, 1866 ; got by Rysdyk's Hambletonian.

Dam, by Harry Clay.

Dam of Harry Clay by imported Bellfounder.

\section{FOUR YEAR OLD MARES.}

No. 1.

RACOLA. br. m., white hind heels, foaled April 22, 1867 ; got by Rysdyk's Hambletenian.

Dam, Brown Pacer. 
No. 2.

ROSETTA. b. m. little white on hind heels, foaled April 22, 1867 ; get by Rysdyk's Hambletonian.

First dam, Grey Rose, by Harris' Hambletonian, by Bishop's Hambletonian, by imported Messenger.

Dam of Harris' Hambletonian was a Messenger mare, imported by Israel Munson, of Boston.

\section{No. 3.}

CALYPSO. b. m., small spot on nuse, white hind ankles white fore heels, white hairs in tail, white spot on near side, foaled July 4, 1867; got by Rysdyk's Hambletonian.

Dam, Kate Smith, by old Abdallah.

\section{No. 4.}

JOSEPHINE. b. m, star, white hind ankles, foaled August 13, 1867 ; got by Rjsdyk's Hambletonian.

Dam, Lucy’Almack, by young Almack, by Abdallah, by Mambrino, by imported Messenger.

\section{No. 5.}

GAY SPANKER. r. m., black points, foaled July 9, 1867 ; got by Rysdyk's Hambletonian.

First dam, Roan Marie.

Second dam, by Abdallah. 
No. 6.

BESS. $b$. m., star and snip, white near ankles, brown spot behind the shoulder, foaled August 16, 1867 ; got by Rysdyk's Hambletonian.

First dam, Jessie Sayre ; by Harry Clay.

Second dara, by Liberty.

\section{No. 7.}

ZENOBIA. br. m. black points, foaled September 18, 1867 ; got by Rysdyk's Hambletonian.

First dam, Belle, by Post's Hambletonian, by Rysdyk's Hambletonian.

Second dam, by Ethiopian Prince, by Wild Air

Third dam, from Ohio.

\section{THREE YEAR OLD FILLIES.}

No. 1.

VIOLET. b. f., black points, foaled March 16, 1868; got by Rysdyk's Hambletonian.

First dam, Sallie Feagles, by Smith's Clay, he by Cassius M. Clay.

Second dam, by Hickory.

Third dam, of Messenger blood. 
No. 2.

ZERLINA. b. f., little white on near hind heel, foaled April 19, 1868 ; got by Rysdyk's Hambletonian.

First dam, Annie Seeley, by Gen'l Gifford.

Second dam, by Old Abdallah.

Third dam, by Tom Thumb.

Fourth dam, Kent mare (dam of Rysdyk's Hambletonian).

\section{No. 3.}

BLOSSOM. b. f., small star, white near hind heel, foaled May

1, 1868 ; got by Rysdyk's Hambletonian.

First dam, Hattie Wood, by Harry Clay.

Second dam, Grandm:other, by Terror.

Harry Clay ; got by Cassius M. Clay, Jr., he by old Cassius M. Clay.

Cassius M. Clay, Jr.

First dam, by Chancellor, son of Mambrino.

Second dam, by Engineer.

Terror, got by American Eclipse.

First dam, Lady Lightfoot, by Sir A rehy.

Second dam, black Maria, by imp. Shark.

Third dam, by imported Clockfast.

Fourth dam, Burwell's Maria, by Regulus.

Fifth đam, Cammilla, by imported Fearnaught.

Six th dam, Calesta, by Forrester.

\section{No. 4.}

QUEEN OF MAY. b. f., white near hind ankle, white off hind heel, foaled May 1, 1868; got by Rysdyk's Hambletonian.

First dam, Lady Overton, by Abdallah.

Second dam, by Mt. Holly, by Mambrino. 
No. 5.

CARRIE. b. f., white hind ankles, foaled May 17, 1868, got by Woburn.

Dam, Jennie Lewis, by Black Bashaw.

No. 6.

STARBEAM. b. f. star, white on lip, white near fore ankle, white hind ankles, foaled June 12, 1868; got by Rysdyk's Hambletonian.

Dam, Montgomery Maid, by American Star.

\section{No. $\%$.}

UNDINE. b. f. white near hind ankle, foaled June 26, 1868 ; got by Rysdyk's Hambletonian.

First dam, Roan Marie.

Second dam, by Abdallah.

No. 8.

br. f., black points, foaled 1868, got by Truesdell's Hambletonian.

First dam, Babbitt Abdallah, by old Abdallah.

Second dam, by Phillips, son of Duroc.

Third dam, by Decatur (thoroughbred) son of

Henry, sister to Abdallah Chief. 


\section{TWO YEAR OLD FILLIES.}

No. 1.

BELLE ROE. ch. f., star, white off hind heel, white near hind ankle, foaled May 2, 1869; got by Idol

First dam, Satinet, by Abdallah Chief (Roe's).

Second dam, by Whistle Jacket.

Third dam, by the Bertholf Horse.

Fourth dam, by old Duroc.

Abdallah Chief (Roe's', by Abdallah.

First dam, by Phillips, by Duroc.

Second dam, by Decatur, son of Henry.

Whistle Jacket, by Mambrino.

Dam, by American Eclipse.

Bertholf Horse, by imported Messenger.

No. 2.

ADA DUROC. b. f., white hind ankles, foaled May 15, 1869; got by Messenger Duroc.

Dam, Montgomery Maid, by American Star.

No. 3.

ZEPHYR. b. f., bluck pointz, foaled May 15, 1869; got by Idol.

First dam, Miss Wansor, by Sir Archy.

Second dam, by Abdallah.

Third dam, by Westchester. 
No. 4.

IDA WEBSTER. b. f., star, white hind heels, foaled May 24,1869 ; got by Rysdyk, he by Rysdyk's Hambletonian.

First dam, Sharpless Abdallah, by Abdallah. Second dam, by Hunter sire of (Flora Temple and De Lancy mare, called Mist).

No. 5.

SUNBEAM. b. f, white near hind heel, foaled June 20 , 1869 ; got by Idol.

Dam, Lady Packer, got by Witherell Messenger, by Winthrop Messenger, by imported Messenger.

No. 6.

MADELINE. b. f., strip in face, white hind ankles, foaled August 6, 1869; got by Abdallah Star.

First dam, Fannie Fiske, by Plough Boy, by L. I. Black Hawk.

Second dam, by old Almack by Mambrino.

No. $\%$.

BOULOTTI. b. f., star and snip, white hind ankles, foaled August 17, 1869; got by Idol.

First dam, Lady Mead, by American Star. Second dam, by Saltram. 


\section{YEARLING FILLIES.}

\section{No. 1.}

ch. f., small star, foaled April 18th, 1870, got by War Eagle.

First dam, Lady Hawkins, by old Abdallah. Second dam, Emily, by Commodore, he by Mambrino.

No. 2.

ch. f., strip in face, white legs, foaled April 21st 1870, got by Messenger Duroc.

First dam, Lady Bellfounder, by Tom Thumb. Second dam, by Crabtree Bellfounder. Third dam, by imported Bellfounder.

No. 3.

b. f., white off hind ankle, foaled April 23, 1870, got by Pierson.

First dam, Bonnie Lassie, by Rysdyk's Hambletonian.

Second dam, by Fox Hunter. 
No. 4.

b. f., star and snip, white hind ankles, foaled May 1, 1870, got by Idol.

First dam, Satinet, by Abdallah chief (Roe's). Second dam, by Whistle Jacket. Third dam, by the Bertholf Horse.

Fourth dam, by old Duroc.

Abdallah Chief (Roe's), by Abdallah.

First dam, by Phillips, son of Duroc.

Second dam, by Decatur (thoroughbred), son of Henry.

Whistle Jacket, by Mambrino:

Dam, by American Eclipse.

Bertholf Horse, by imported Messenger.

\section{No. 5.}

b. f., black points, foaled May 2, 1870, got by Messenger Duroc.

First dam, Lady Pierson.

\section{No. 6.}

b. f., white hind ankles, white near fore heel, foaled May 6, 1870, got by Rysdyk's Hambletonian.

First dam, Lucy Almack, by young Almack, by old Almack, by Mambrino, by imported Messenger. 
No. 7.

LADY BEIMONT. br. f. strip in face, white near ankles. foaled May 7,1870 , got by Rysdyk's Hambletonian.

First dam, Kate (Bruno's Dam), by Bellaire, the sire of Red Bird.

Second dam, a pacing mare of Comic blood.

\section{No. 8.}

b. f. white hind heels, foaled May 10, 1870, got by Idol.

First dam, Susie Roberts, by New York Black Hawk.

Second dam, the well known mare of Messenger blood, owned and driven by Captain Roberts, of New York.

\section{No. 9.}

ch.f. strip in face, white hind legs, foaled May

15, 18i0, got by Messenger Duroc.

First dam, Coquette, by Wild Irishman.

No. 10.

blk. f. white off hind heel, foaled June 33, 1870, got by Messenger Duroc.

First ảam, Mildred, by Rysdyk's Hambletonian.

Second dam, Linda (the old trotting mare). 


\section{No. 11.}

b. f., star, spoc on nose, black points, foaled June 9, 1870, got by Pierson.

First dam, Amanda, by Rysdyk's Hambletonian.

Second dam, Lady Cardinal, by Cardinal. Third dam, by One Eyed Hunter.

No. 12.

b. f., black points, foaled June 10, 1870, got by Rysdyk's Hambletonian

First dam, Jennie Lewis, by Black Bashaw.

No. 13.

b. f, star, white hind heels, foaled June 18, 1870 , got by Messenger Duroc.

Dam Stella.

No. 14.

b. f., star, white hind heels, foaled June 18, 1870 , got by Imperial, by Rysdyk's Hambletonian.

First dam, Smith's Star, by American Star.

Second dam, by L. I. Black Hawk.

\section{No. 15.}

br. f., star and snip, white hind heels, foaled Jure 21, 1870, got by Knickerbocker.

First dam, Nellie Sayres. 


\section{No. 16.}

blk. f., white off hind heel, foaled June 23, 1870, got by Messenger Duroc.

First dam, Green Mountain Maid, by Harry Clay.

Second dam, by Lexington,

Dam/ of Harry Clay, by imported Bellfounder

\section{No. 17.}

b. f., star, white off hind heel, foaled July 12, 1870, got by Rysdyk's Hambletonian.

First dam, Babbitt Abdallah, by old Abdallah.

Second dam, by Phillips, son of Duroc.

Third dam, by Decatur (thoroughbred), son of Henry, sister to Abdallah Chief

\section{No. 18.}

blk. f., white heels, foaled July 26, 1870, got by Messenger Duroc.

First dam, Mattie Bull.

Second dam, by Obio Eclipse.

Third dam, by Post Boy.

\section{No. 19.}

br. f., star and snip, white off hind heel, foaled August 2, 1870, got by Waverley.

First dam, Lady Mead, by American Star.

Second dam, by Saltram. 
No. 20.

br f., white off fore ankle, white off hind beel, foaled August 3, 1870, got by Waverley. First dam, Lady Farr, by old Abdallah.

\section{N O T I C E.}

I am now ready to offer a portion of my Stock at Private Sale during the Year. CHARLES BACKMAN. 


\section{872 .}

\section{Trotting Stock,}

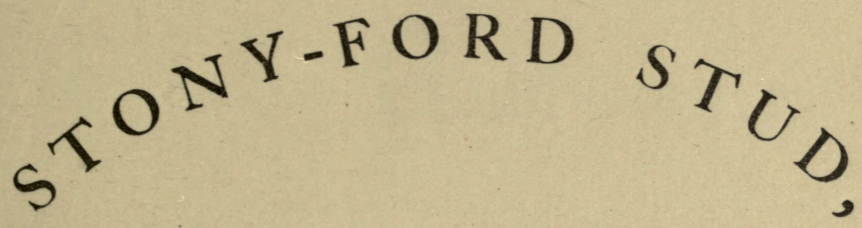

ORANGE COUNTY,

$\mathrm{N}_{\mathrm{EW}} \mathrm{Y}_{\mathrm{ORK}}$,

CHARLES BACKMAN.

NEW YORK :

Henry Spear, Printer and Stationer, $8: 2$ W Wtl Street. 



\section{PEDIGREE}

OF

Rysdyk's Hambletonian.

"Sired by Old Abdallah, he by Mambrino, he by imported Messenger. His dam was the Charles Kent Mare, by imported Rellfounder; grandam, Old One Eye, by old Hambletonian, and he by imported Messenger, and his dam also by imported Messenger; and the dam of Old One Eye was by imported. Messenger." 


\section{STONY FORD STUD, I 872 .}

The following Ssallions are offered to a limited number of Mares Each in addition to my own at One Hundred Dollars (in advance) for the season which will commence on the first day of April and end the first day of November.

ABDALLAH STAR.—Ch. s. 15.3, foaled June 12, 1863, got by American Star, Jr., first dam, Curry Abdallah, by Abdallah; $2 \mathrm{~d}$ dam by imp. Bellfounder; $3 \mathrm{~d}$ dam by

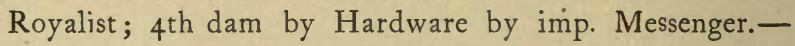
American Star, Jr., got by Edmund Seely's American Star, dam by Friday, he by imp. Trustee.

IDOL. -b. s. $15.21 \frac{1}{2}$, foaled June 9, 1864, got by Rysdyk's Hambletonian, 1st dam Hattie Wood, by Harry Clay; $2 \mathrm{~d}$ dam Grandmother, by Terror; Terror by American Eclipse.

MESSENGER DUROC.-b. s. 16 hands, foaled June 3, 1865 got by Rysdyk's Hambletonian; 1st dam Satinet, by Roe's Abdallah Chief; $2 \mathrm{~d}$ dam by Whistle Jacket; $3 \mathrm{~d}$ dam by the Bertholf horse; $4^{\text {th }}$ dam by old Duroc. Roe's Abdallah Chief, by Abdallah; 1 st dam by Phillips' son of Duroc; 2d dam by Decatur, son of Henry. Whistle Jacket by Mambrino, first dam by American Eclipse.Bertholf horse by imp. Messenger.

BOLTON.-b. s. 16 hands, foaled June 13, 1867, got by Rysdyk's Hambletonian, 1st dam Montgomery Maid, by American Star. 


\section{Stony-Ford Stud.}

\section{STALLIONS.}

ABDALLAH ch. s. 15.3 hands, small star, white off hind STAR. ankle, foaled June 12, 1863; got by American Star, Jr.

First dam, Curry Abdallah, by Abdallah. Second dam, by imported Bellfounder. Third dam, by Royalist.

Fourth dam, by Hardware, by imp. Messenger. American Star, Jr. ; got by American Star. Dam by Friday, he by imported Trustee. 
ID0L. b. s., $15.2 \frac{1}{2}$ hands, white near fore heel, white hind ankles, foaled June 9, 1864 ; got by Rysdyk's Hambletonian.

First dam, Hattie Wood, by Harry Clay.

Second dam, Grandmother, by Terror.

Harry Clay; by Cassius M. Clay, Jr.

Dam by imported Bellfounder.

Cassius M. Clay, Jr., by old Cassius M. Clay.

First dam by Chancellor, son of Mambrino.

Second dam, by Engineer.

Terror; got by American Eclipse.

First dam, Lady Lightfoot, by Sir Archy.

8econd dam, Black Maria, by imported Shark.

Third dam, by imported Clockfast.

Fourth dam, Burwell's Maria, by Regulus.

Fifth dam, Camilla, by imported Fearnaught.

Sixth dam, Calesta, by Forrester.

PIERSON. b. s. 16 hands, white hind heels, foaled May, 1864 ; got by Rysdyk's Hambletonian.

First dam, by Cassius M. Clay, Jr.

Second dam, by Black Bird.

Cassius M. Clay, Jr., by Cassius M. Clay. Black Bird, by Roebuck. 
MESSENGER b, s., 16 hands, white hind ankles, foaled DUR0C. June 3, 1865; got by Rysdyk's Hambletonian. First danu, Satinet, by Abdallah Chief (Roe's.)

Second dam, by Whistle Jacket.

Third dam, by the Bertholf Horse.

Fourth dam, by old Duroc.

Abdallah Chief (Roe's), by Abdallah.

First dam, by Phillips, by son of Duroc.

Second dam, by Decatur (thoroughbred), son of Henry.

Whistle Jacket, by Mambrino.

First dam, by American Eclipse.

Bertholf Horse ; by imported Messenger.

STONY-FORD. br. s., 15.1 hands, little white on near hind heel, foaled May 27, 1866; got by Rysdyk's Hambletonian.

First dam, Annie Seely, by Gen'l Gifford.

Second dam, by old Abdallah.

Third dam, by Tom Thumb.

Fourth dam, Kent Mare, dam of Rysdyk's Hambletonian.

WAVERLEY. br. s., 15.3 hands, star, white hind ankles, white off fore heel, foaled October 9, 1866; got by Rysdyk's Hambletonian.

First dam, Susie Roberts, by N. Y. Black Hawk.

Second dam, the well known mare of Messenger blood, owned and driven by Captain Roberts, of New York.

N. Y. Black Hawk, by L. I. Black Hawk. 


\section{Five Year Old Stallions.}

No. 1.

D AUNTLESS. b. s., small star, black points, foaled April 1, 1867 ; got by Rysdyk's Hambletonian.

First dam, Sallie Feagles, by Smith's Clay.

Second dam, by Hickory.

Third dam, of Messenger Blood. Smith's Clay, by Cassius M. Clay, Jr.

No. 2.

MENELAUS. br. s., star, white hind ankles dotted with black, foaled April 14, 1867; got by Rysdyk's Hambletonian.

First dam, Jessie Bull, by L. I. Black Hawk. Second dam of Messenger Blood.

No. 3.

VICTOR VON b. s., white hind heels, foaled April 19, 1867 ;

BISMARCK. got by Rysdyk's Hambletonian.

Full Brother to First dam, Hattie Wood, by Harry Clay.

Second dam, Grandmother, by Terror.

No. 4.

AITCHESON. b s., star, white near hind ankle, foaled.June 13, 1867 ; got by Rysdyk's Hambletonian.

First dam, Lady Van Buren, by Red Bird. Second dam, young Bellfounder, by imported Bellfounder. 


\section{No. 5.}

BOLTON. b. s. white off hind heel, foaled June 13, 1867 ; got by Rysdyk's Hambletonian.

First dam, Montgomery Maid, by American Star.

No. 6.

EXCHANGE. g. s., star and snip, white hind ankles, foaled July 4, 1867; got by Rysdyk's Hambletonian. Dam, Lady Swazey, by Harris' Hambletonian, by Bishop's Hambletonian, by imported Messenger.

Dam of Harris' Hambletonian - was a Messenger mare, imported by Israel Munson, of Boston.

No. 7 .

KENILWORTH. br. s., black points, foaled Sept 10, 1867 ; got by Rysdyk's Hambletonian.

First dam, Susie Roberts, by N. Y. Black Hawk.

Second dam, the well-known mare of Messenger blood, owned and driven by Captain Roberts, of New York.

N. Y. Black Hawk; got by L. I. Black Hawk. 


\section{SIX YEAR OLD GELDING.}

ISLAND BOY. b. g., 15.2 hands, white off heels, foaled 1866, got by Hetzell Hambletonian.

First dam, Lady Durland, by American Star.

Second dam, by Nigger Lance, he by Old Lance.

\section{FOUR YEAR OLD GELDING.}

SILVER HEEL. b. g., white near hind heel, foaled May 9, 1868 ; got by Rysdyk's Hambletonian.

First dam, Rosa Lee, by Harris' Hambletonian, by Bishop's Hambletonian, by imp. Messenger.

The dam of Harris' Hambletonian was a Messenger mare, imp. by Israel Munson, of Boston. 


\section{THREE YEAR OLD GELDINGS.}

No. 1.

LUCKNOW. b. g., has star and snip, white near hind heel, white off fore heel, foaled May 11, 1869 ; got by Idol.

Dam, Grandmother, by Terror. See "Pedigree of Idol"

No. 2.

PICKWICK. br. g., star and snip, white hind ankles, little white on near fore heel, foaled June 9, 1869; got by Idol.

First dam, Lady Brown, by Américan Star.

Second dam, by Nigger Lance, he by old Lance [a thoroughbred].

See Pedigree of Idol.

No. 3.

PROSPER0. blk. g., white hind heels, foaled July 12, 1869 ; got by Messenger Duroc.

Firśt dam, Green Mountain Maid, by Harry Clay.

Second dam, by Lexington. 


\section{FOUR YEAR OLD COLTS.}

No. 1.

TWILIGHT. b. c. white hind heels, foaled April, 20, 1868; got by Rysdyls's Hambletonian.

First dam, Mary Hulse, by American Star. Second dam, of Messenger blood.

No. 2.

ELECTIONEER. b. c., white hind heels, foaled May 2, 1868, got by Rysdyk's Hambletonian.

First dam, Green Mountain Maid, by Harry Clay.

Second dam, by Lexington.

Dam of Harry Clay, by imp. Bellfounder.

No. 3.

CUYLER. b. c., star near white hind ankle, foaled May 13, 1868 ; got by Rysdyk's Hambletonian.

First dam, Grey Rose, by Harris' Hambletonian, by Bishop's Hambletonian, by imp. Messenger.

Dam of Harris' Hambletonian was a Messenger mare, imported by Israel Munson, of Boston. 
No. 4.

TROUBLE. b. c, white hind ankles, foaled May 22, 1868 ; got by Rysdyk's Hambletonian.

First dam, Lady Hopkins, by a son of Hill's Black Hawk.

Second dam, by Harris' Hambletonian.

Dam of Son of Hill's Black Hawk, by Harris' Hambletonian.

No. 5.

DIRECT0R. b. c, small star, white hind ankles, foaled June 9, 1868 ; got by Rysdyk's Hambletonian.

First dam, Lady Bellfounder, by Tom Thumb. Second dam, by Crabtree Bellfounder.

- Third dam, by imported Bellfounder.

No. 6.

MAMBRINंO b. c., white hind heels, white near foot, foaled STAR. 1868; got by Mambrino Pilot.

First dam, Lady Dexter, by Rysdyk's Hambletonian.

Second dam (Dexter's dam), by American Star.

Mambrino Pilot, got by Mambrino Chief (the sire of Lady Thorn).

Dam by Alexander's Pilot, Jr., the sire of John Morgan. 


\section{THREE YEAR OLD COLTS.}

No. 1.

TRIUMPH. b. c., white hind heels, foaled April 26, 1869 ; got by Idol.

First dam, Rosa Lee, by Harris' Hambletonian, by Bishop's Hambletonian, by imp. Messenger.

The dam of Harris' Hambletonian was a Mes. senger mare, imported by Israel Munson, of Boston.

No. 2.

GARDNER. ch. c., star, white hind heels, foaled Auguist 19, 1869 ; got by Messenger Duroc.

First dam, Lady Overton, by old Abdallah.

Second dam, by Mount Holly.

Mount Holly, by old Messenger. 
No. 3.

GEORGE ch. c. star and snip, white hind heels, white GARDINER. near fore heel, foaled September 9, 1869; got by Messenger Duroc.

First dam Hattie Wood, by Harry Clay.

Second dam, Grandmother, by Terror.

Harry Clay; got by Cassius M. Clay, Jr.

Dam, by imp. Bellfounder.

Cassius M. Clay, Jr, by old Cassius M. Clay.

First dam, by Chancellor, son of Mambrino.

Second dam, by Engineer.

Terror; got by American Eclipse.

First dam, Lady Lightfoot, by Sir Archy.

Second dam, Black Maria, by imp. Shark.

Third dam, by imported Clockfast.

Fourth dam, Burwell's Maria, by Regulus.

Fifth dam, Cammilla, by imported Fearnaught.

Sixth dam, Calesta, by Forrester.

\section{TWO YEAR OLD COLTS.}

\section{No. 1.}

b. c., black points, foaled April 18th, 1860 ; got by Messenger Duroc.

First dam, Trustus Webb.

Second dam by Trustee. 
No. 2. .

b. c., strip in face, white off fore ankle, white hind ankles, foaled April 24th, 1870 ; got by Pierson First dam, Zuleika, by Brooks' Black Hawk, by L. I. Black Hawk.

Second dam, by Mambrino Chief.

\section{No. 3.}

b. c., white hind ankles, foaled May 5th, 1870, got by Pierson.

First dam, Alice Van Duzer, by American Star.

Second dam by old Messenger Duroc.

Third dam by Paymaster.

No. 4.

ch. c., star, white hind ankles, foaled May 21st, 1870, got by Messenger Duroc.

First dam, Grey Rose, by Harris' Hambletonian.

Dam of Harris' Hambletonian was a Messengger mare, imported by Israel Munson, of Boston. 
No. 5 .

BELMONT. b. c., white hind heels, foaled May 22d, 1870, got by Rysdyk's Hambletonian.

First dam, Miss Wansor. by Sir Archy.

Second dam by Abdallah.

Third dam by Westchester.

No. 6.

b. c., snip on nose, white fore heels, white hind ankles, foaled June 2d, 1870, got by Messenger Duroc.

First dam, Lady Cardinal, by Cardinal.

Second dam, by imported Bellfounder.

No. $\%$.

blk c., white near fore heel, white hind heels, foaled June 5th, 1870 ; got by Messenger Duroc.

First dam Montgomery Maid, by American Star.

No. 8.

ch. c., star, white hind heels, foaled June 25th, 1870 ; got by Messenger Duroc.

First dam, Lady Nodine, by Rysdyk's Hambletonian.

Second dam, Montgomery Maid, by American Star. 
No. 9.

b. c., star, white off hind heel, white near hind ankle, white near fore leg; foaled July 3d, 1870 ; yot by Rysdyk's Hambletonian.

First dam, Fannie Fiske, by Plough Boy, by . L. I. Black Hawk.

Second dam, by old Almack, by Mambrino.

No. 10.

b. c., white hind heels, foaled July 6th, 1870 ; got by Pierson.

First dam, Roan Marie, by horse from Canada. Second dam by Abdallah.

\section{No. 11.}

b. c, star, white hind ankles, foaled July 14th, 1870; got by Messenger Duroc.

First dam, Virgo, by Abdallah Chief (Roe's.)

Second dam, by Whistle Jacket.

Third dam, by the Bertholf Horse.

Fourth dam, by old Duroc. .

Abdallah Chief (Roe's), by Abdallah.

First dam, by Phillips, by son of Duroc.

Second dam, by Decatur (thoroughbred], son of Henry.

Whistle Jacket, by Mambrino.

First dam, by American Eiclipse.

Bertholf Horse, by imported Messenger. 


\section{YEARLING COLTS.}

No. 1.

b. c., star and snip, white hind ankles, foaled April 14th, 1871, got by Waverly.

First dam, Zuleika, by Brooks Black Hawk.

Second dam, by Mambrino Paymaster.

\section{No. 2.}

blk. c., star, white off fore foot, white hind ankles, foaled April 21st, 187 i, got by Messunger Duroc, dam, Nellie Wilmarth, by Kemble Jackson.

\section{No. 3.}

b. c., star in forehead, foaled A pril 29th, 1871, got by Waverly.

First dam, Lady Pierson, by. Cassius M. Clay, Jr.

Second dam by imported Diamond.

\section{No. 4.}

ch. c., strip in face, white near hind ankle, foaled May 5th, 1871, got by Abdallah Star.

First dam, Lady Rockafellow, by American Star.

Second dam by Bay Richmond.

Third dam by Gilbert Oliver's Jackson, by son of Messenger. 
No. 5.

ch. c., white hind ankles, foaled May 15th, 1871, got by Idol.

First dam, Emma Hoyt, by American Star.

Second dam by Ohio Eclipse.

Third dam of Messenger blood.

No. 6.

b. c., star, white hind ankles, foaled May 18th, 1871, got by Messenger Duroc.

First dam, Mildred, by Rysdyk's Hambletonian.

Second dam, Linden (the old trotting mare).

No. \%.

ch. c., star and snip, white fore ankles, white near hind ankle, foaled May 28th, 1871, got by Messenger Duroc.

Dam, Glencoe Maid.

No. 8 .

b. c., star and snip, white hind ankles, foaled May 29 th, 1871, got by Idol.

First dam, Belle of Richmond, by Rysdyk's Hambletonian.

Second dam, old Belle of Richmond, by Grey Norman. 
No. 9.

br. c., white hind heels, foaled May 30th, 1871, got by Messenger Duroc.

First dam, Sharpless Abdallạh, by old Abdallah.

Second dam by Hunter.

No. 10.

CARL S. BURR. b. c., star, white near fore heel, white near hind heel, small white spot on off hind heel, foaled Yay 31st, 1871, got by Rysdyk's Hambletonian.

Dam, Kate, (Bruno's dam).

\section{No. 11.}

b. c., white near fore foot, white hind ankles foaled June 5th, 1871, got by Messenger Duroc. Dam, Lucy Almack, by young Almack, by old Almack, by Mambrino, by imported Messenger.

\section{No. 12.}

ch. c., star, white hind legs, foaled June 8th, 1871, got by Messenger Duroc.

First dam, Emma Mills by American Star. Second dam by Alexander W., by Alexander, (thoroughbred from Virginia.)

\section{No. 13.}

ch. c., strip in face, foaled June 10th, 1871, got by Messenger Duroc.

First dam, Green Mountain Maid, by Harry Clay.

Second dam by Lexington. 


\section{No. 14.}

cb. c, white hind ankles, foaled June 17th, 1871, got by Messenger Duroc.

First dam, Susie Roberts, by N. Y. Black Hawk.

Second dam, the well-known mare of Messenger blood, owned and driven by Captain Roberts of New York.

No. 15.

blk c.. star, white hind ankles, foaled June 24 th, 1871, got by Messenger Duroc.

Dam, Mentgomery Maid, by American Star.

No. 16.

b. c., star, black points, foaled June 25th, 1871, got by Messenger Duroc.

Dam Lady Packer, by Witherell's Messenger, by Winthrop's Messenger, by Bush Messenger, by imported Messenger.

\section{No. $1 \%$.}

b. c., star and snip, white hind heels, foaled July 16th, 1871, got by Abdallah Star.

First dam, Alice Van Duzer, by American Star.

Second dam by Messenger Duroc.

Third dam by Paymaster.

\section{No. 18.}

b. c., star, white spot on off hind heel, foaled July 18th, 1871, got by Rysdyk's Hambletonian.

First dam, Kate Seely, by Walden's Messenger, by Wildair, he by son of imported Messenger.

Second dam by Mambrino, by imported Messenger. 
No. 19.

b. c., white hind ankles, foaled July 20 th, 1871 , got by Messenger Duroc.

First dam, Babbitt Abdallah by Old Abdallah.

Second dam, by Phillips, son of Duroc.

Third dam, by Decatur, (thoroughbred,) son of Henry, sister to Abdallah Chief.

No. 20.

b. c., strip in forehead, white off hind ankle, foaled August 5th, 1871, got by Messenger Duroc.

Dam, Lady Ellis, by Alexander's Abdallah, (formerly Edsell's Hambletonian.)

\section{No. 21.}

b. c., strip in face, white hind ankles, foaled $\mathrm{Au}$ gust 14th, 1871, got by Messenger Duroc.

First dam, Lady Brown, by American Star

Second dam, by Nigger Lance, by old Lance a thoroughbred.

\section{No. 22.}

b. c., star and snip, white off fore foot, white hind ankles, got by Imperial.

First dam, Mary Sanford, by PPlunkett Horse.

Second dam, Lady Sanford, by American Star. 


\section{BROOD MARES.}

\section{No. $\dot{1}$.}

ROAN MARIE. rn m., 16 hands, foaled 1853, got by a horse from Canada.

Dam, by Abdallah.

No. 2.

GREY ROSE. gr. m. $15 \frac{1}{2}$ hands got by Harris' Hambletonian, he by Bishop's Hambletonian, by imported Messenger.

Dam of Harris' Hambletonian was a Messenger mare, imported by Israel Munson, of Boston.

No. 3.

BELI.E. ch. m. 15.3 hands, star and snip, little white on hind heels, foaled 1861, got by Post's Hambletonian, by Rysdyk's Hambletonian.

First dam, by Ethiopian Prince, by Wildair.

Second dam, from Ohio.

Post's Hambletonian; got by Rysdyk's Hambletonian.

First dam, said to be by Bellfounder.

Second dam, said to be of Messenger breed.

No. 4.

ALICE VAN DUZER. blk. m. 15 hands, strip in face, white hind ankles, white near fore heel, foaled May, 1852, got by American Star.

First dam, by old Messenger Duroc.

Second dam, by Paymaster. 
No. 5.

KATE SEELEY. b. m. 15.3 hands, white near hind heel,

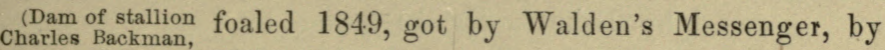
owned by
J. G. Wood, Wildair, he by son of imp. Messenger. w.Millbury, Mass.) First dam, by Mambrino, by imp. Messenger.

No. 6.

LADY BROWN. br. m. 15 hands, star and snip, white off (Dam of Gauntlet hind heel, foaled 1861, got by American Star. wilkins Micawber.) First dam by Nigger Lance, by old Lance, a thoroughbred.

\section{No. $\%$.}

LADY MEAD. b. m. 16 hands, star and snip, white near (Dam of Nettle.) hind ankle, foaled 1856, got by American Star. Dam, by Saltram.

\section{No. 8.}

LIZZIE WALKER. ch. m. 15 hands, star and snip, white (Dam of Startle.) hind legs, white off fore heel, got by American Star.

First dam, by old Hickory, Hickory by Whip, Whip by Seymour's Wildair, Wildair by Fearnaught.

\section{No. 9.}

LADY BELLFOUNDER. ch. m. 15.2 hands, strip in face, "Wnown as the , white near hind leg up to hock, white off hind (Dam of Bellona.) ankle, foaled 1856, got by Tom Thumb.

First dam, by Crabtree's Bellfounder.

Second dam, by imported Bellfounder. 
No. 10.

ROSA LEE. gr. m. 15 hands, got by Harris' Hambletonian, by Bishop's Hambletonian, by imp. Messenger. Dam of Harris' Hambletonian was a Messenger mare, imported by Israel Munson of Boston.

\section{No. 11.}

SALLIE FEAGLES. br. m. $15.2 \frac{1}{2}$ hands, black points, foaled 1854, got by Smith's Clay, by Cassius M. Clay, Jr.

First dam, by Hickory, by Mambrino, by imp. Messenger

Second dam; of Messenger blood.

\section{No. 12.}

LUCY ALMACK. b. m. 15.2 hands, star, white off hind ankle, white near fore heel, foaled 1857, got by young Almack, by old Almack, by Mambrino, by imported Messenger.

\section{No. 13.}

MAGGIE JONES. ch. m. 15.1 hands, star, little white on near hind heel, foaled 1856, got by American Star.

Dam, by Mambrino Chief. 


\section{No. 14.}

ANNIE SEELY. b m. $15.1 \frac{1}{2}$ hands, black points, foaled 1858 , got by General Clifford.

First dam, by old Abdallah.

Second dam, by Tom Thumb.

-Third dam, Kent mare, dam of Rysdyk's Hambletonian.

Gen'l Gifford; got by Gifford Morgan, he by Green Mountain Morgan

Second dam, by Tom Thumb, was the dam of Green's Bashaw, sire of Faucett's Bashaw and Sam Kirkwood.

\section{No. 15.}

LADY DURLAND. ch. m. 15 hands, star and snip, foaled 1860, got by Americau Star.

Dam, by Nigger Lauce, he by old Lance.

\section{No. 16.}

SUSIE ROBERTS. b. m. $15.1 \frac{1}{2}$ hands, black points, foaled 1853, got by .N. Y. Black Hawk, he by L. I. Black Hawk.

Dam, the well-known mare of Messenger blood, owned and driven by Captain Roberts, of New York.

\section{No. 17.}

MISS WANSOR. b. m. 16.1 hands, black points, foaled 1860, got by Sir Archy.

First dam, by Abdallah.

Second dam, by Westchester. 
No. 18.

LADY HAW KINS. b. m. 15.2 hands, white off hind heel, foaled 1850, got by old Abdallah.

Dam, Emily, by Commodore, he by Mambrino.

No. 19.

BELLE OF RICHMOND, gr. m. 15.1 hands, white near hind ankle, foaled 1861, got by Rysdyk's Hambletonian.

Dam, old Belle of Richmond, by Grey Norman.

No. 20.

LADY PACKER. gr. m. 15.2 hands, black points, foaled 1860, got by Witherell's Messenger, by Winthrop's Messenger, by Bush Messenger, by imported Messenger.

\section{No. 21.}

AGNES BOYLE. b. m. 15.3 hands, strip in face, white Full Sister to
Post's Ham- near ankles, foaled 1856, got by Rysdyk's Hambletonian. bletonian

First dam said to be by Bellfounder.

Second dam, said to be of Messenger blood.

No. 22.

MISTRESS HENY0N. ch. m. 15.2 hands, star and strip white hind legs, white near fore heel, got by Abdallah Chief [Roe's,] by old Abdallah.

Dam, by Sir Henry. 
No. 23.

MILDRED. b. m. 15 hands, star, white off hind ankle, foaled 1858, got by Rysdyk's Hambletonian.

Dam, Linda, the old trotting mare

\section{No. 24.}

GREEN MOUNTAIN MAID. br. m. 15 hauds, star, white hind ankles, foaled 1862, got by Harry Clay.

Dam, by Lexington.

Dam, of Harry Clay, by imported Bellfounder.

No. 25.

KITTY MILLS. b m. 15.1 hands, snip in face, black points, got by American Star.

Dam, by Wildair.

No. 26.

LADY OVERTON. ch. m. $15.2 \frac{1}{2}$ hands, strip in face, white off hind ankle, foaled 1851, got by old Abdallah. Dam, by Mt. Holly, by old Messenger.

No. $2 \%$.

NELLIE WILMARTH. br. m. 15 hands, star, white hind heels, got by Kémble Jackson. 
No. 28.

SPY. br. m. 15.2 hands, strip in face, spot on nose, white off hind heel, got by Bott's Revenue.

Dam by Red Eye, by Boston.

\section{No. 29.}

FANNIE FISKE. b. m. 15.2 hands, white near hind leg, white fore heel, foaled 1853, got by Plough Boy, by L. I. Black Hawk.

Dam, by old Almack, by Mambrino

\section{No. 30.}

LADY HOPKINS. b. m. 15.3 hands, white off hind heel, foaled 1856, got by son of Hill's Black Hawk.

Dam, by Harris' Hambletonian.

\section{No. 31.}

VIRG0. blk. m. 15.1 hands, white near hind heel, foaled 1856, got by Abdallah Chief [Roe's].

First dam, by Whistle Jacket.

Second dam, by the Bertholf horse.

Third dam, by old Duroc.

Abdallah Chief (Roe's); by Abdallah.

First dam, by Philips, by son of Duroc.

Second dam, by Decatur, [thoroughbred], son of Henry.

Whistle Jacket, by Mambrino.

First dam by American Eclipse.

Bertholf horse by imp. Messenger. 
No. 32.

MARY HULSE. b. m. 15 hands, white hind ankles, foaled 1860, got by American Star.

Dam of Messenger blood.

\section{No. 33.}

FANNIE STAR. b. m. 15.2 hands, black points, foaled 1860, got by American Star.

Dam by old Abdallah.

\section{No. 34.}

TRUSTUS WEBB. b. m. 15.2 hands, black points, foaled 1860, got by American Star.

Dam by Trustee.

\section{No. 35 .}

CURRY ABDALLAH. ch. m. 16 hands, star and snip, $\left.\begin{array}{c}\text { (Dam of } \\ \text { Abdallah Star.) }\end{array}\right)$ white hind heels, foaled 1847, got by old Abdallah.

First dam, by imported Bellfounder.

Second dam, by Royalist.

Third dam by Hardware, by imp. Messenger.

\section{No. 36.}

SHARPLESS ABDALLAH. br. m. 15.1 hands, white off hind heel, got by old Abdallah.

Dam by Hunter, (sire of Flora Temple and the DeLancy mare called Mist). 
No. $3 \%$.

LADY PIERSON. b. m. 16 hands, star and snip, foaled 1853, got by Cassius M. Clay, Jr.

First dam by imported Diamond.

Second dam, a running mare, said to be thoroughbred.

- No. 38.

LADY NODINE. br. m. 15.1 hands, white hind heels, foaled 1863, got by Rysdyk's Hambletonian.

Dam, Montgomery Maid, by American Star.

No. 39.

ZULEIKA. ch. m. $15.2 \frac{1}{2}$ hands, star, spot on nose, foaled 1857, got by Brooks' Black Hawk, by L. I. Black Hawk.

Dam, by Mambrino Paymaster, by Mambrino Chief.

No. 40.

LADY CARDINA L. blk. m. 15.1 hands, star and snip, white legs, foaled 1863, got by Cardinal.

Dam, by imported Bellfounder. 
No. 41.

MATTIE. b. m. 15.2 hands, black points, foaled 1866, got (Dam of Elander by Rysdyk's Hambletonian.
H. Sherman.)

First dam, Lady Earle, by Chauncey Green.

Second dam, by Judson's Hambletonian.

Third dam by Harris' Hambletonian.

Fourth dam, by Judson's Hambletonian.

Fifth dam, by Harris' Hambletonian.

Chauncey Green, by Hale's Morgan.

Dam, by Judson's Hambletonian.

\section{No. 42.}

BONNIE LASSIE. b. m. $15.1 \frac{1}{2}$ hands, star and snip, white near hind heel, foaled 1865, got by Rysdyk's Hambletonian.

Dam, by Fox Hunter.

\section{No. 43.}

LADY DEXTER. b. m. 15 hands, strip in face, white legs, (Full Sister of foaled 1861, got by Rysdyk's Hambletonian.
Dexter.)

Dam, by American Star.

\section{No. 44.}

EMMA MILLS. ch. m. 15.1 hands, strip in face, white near "Independent.") hind heel, foaled 1853, got by American Star. Dam, by Alexander W., by Alexander (thoroughbred from Virginia.) 


\section{No. 45.}

LEDGER GIRL. br. m 15.1 hands, small star, black points, foaled 1863, got hy Rysdyk's Hambletonian.

Dam, by son of Barney Honry.

No. 46.

GLENCOE MAID. ch. m. 15.1 hands, strip in face, near white hind ankle, foaled 1855, got by son of Glencoe.

No. $4 \%$.

KATE. blk. m. 143 hands, strip in face, white off hind (Bruno's Dam.) ankle, white near hind heel, white near fore ankle, foaled 1852, got by Bellaire, the sire of Red Bird.

Dam, a pacing mare, of Comic blood.

No. 48.

STELLA. b. $m 11 \frac{1}{2}$ hands, strip in face, white near fore heel, white ncar hind ankle.

No. 49.

LADY FALLIS. b. m. 15.3 hands, strip in face, foaled (Dam of Socrates.) 1859, got by American Star.

Dam, by L. I. Black Hawh. 
No. 50.

WELLS' STAR. br. m. 15.1 hands, star and snip, white off hind heel, foaled 1856, got by American Star. Dam, by Bertrand, by Sir Solomon, by Sir Archy.

\section{No. 51.}

WAVERLY HOWLAND. b. m. $15 \frac{1}{2}$ hands. star and snip, white near hind heel, foaled got by Ameri' can Star.

First dam by Black Bird.

Black Bird by American Eclipse.

No. 52.

NEILLIE SAYRES. br. m. 151 hands, star, white off hind heel, foaled got by American Star.

Dam, by Hickory.

\section{No. 53.}

EMMA HOYT. eh. m. $15 \frac{1}{2}$ hands, star and snip, white near hind heel, foaled 1859, got by American Star.

First dam by Ohio Eclipse.

Second dam, a mare of Messenger blood.

No. 54.

HATTIE HOGAN. br. m. 150.3 hands, star and snip, white hind heels, foaled 1867 , got by Harry Clay.

First dam, by American Star.

Second dam, by Hickory. 
No. 55.

ADELE. b. m. $15 \frac{1}{2}$ hands, star and snip, white hind ankles, white near fore heel, foaled 1867, got by Rysdyk's Hambletonian.

Dam, Lady Franklin, owned by David Bonner, Esq.

No. 56.

LADY GA VIN. br m. 15.1 hands, star, white hind heels, foaled got by Rysdyk's Hambletonian.

No. $5 \%$.

JANE MURRAY. g. m. 15.1 hands, foaled 1853.

[Dam of Lottery.]

No. 58.

LADY IRWIN. b. m. 153 hands, spot on nose, white near

hind ankle, white near fore heel, foaled

got by Rysdyk's Hambletonian.

First dam, by Abdallah Chief (Roe's). 
No. 59.

KITTIE HARKER. b. m. 15.2 hands, strip in face, white off hind heel, white spot on off fore heel, foaled got by Ethan Allen.

First dam, Martha, by Abdallah.

Second dam, by young Bellfounder.

Third dam, by Corn Cracker.

Fourth dam, by Hickory.

Abdallah, by Mambrino.

Dam, Smazonia, by imported Messenger.

Young Bellfounder, by imported Bellfounder.

Dam by Wildair.

Corn Cracker, b. h. foaled 1838 , by imported Tranby.

Dam, Mary Haxwell, by Moses.

No. 60.

FAIRY. b. m. 15.2 hands, white hind ankles, foaled 1865, got by Rysdyk's Hambletonian.

First dam, Emma Mills, by American Star. Second dam, by Alexander W., by Alexander (thoroughbred from Virginia). 


\section{No. 61.}

BLOSSOM. b. m. 15.2 hands, small star, white near hind (Full sister to heel, foaled May 1st, 1868, got by Rysdyk's Hambletonian.

First dam, Hatty Woods by Harry Clay.

Second dam, Grandmother by Terror.

Harry Clay by Cassius M. Clay, Jr.

Dam by imported Bellfounder.

Cassius M. Clay, Jr., by old Cassius M. Clay.

First dam, by Chancellor, son of Mambrino.

Second dam, by Engineer.

Terror, got by American Eclipse.

First dam Lady Lightfoot, by Sir Archy.

Second dam, Black Maria, by imported Shark.

Third dam, by imported Clockfast.

Fourth dam. Burwell's Maria, by Regulus.

Fifth dam, Camilla, by imported Fearnaught.

Sixth dam, Calesta, by Forrester.

No. 62.

LADY DAVIES. ch. m. 15.2 hands, strip in face, white hind legs, foaled got by Long Island Black Hawk.

No. 63.

LADY GALATIOY. ch. m. 15.3 hands, star, white near hind ankle, foaled got by American Star. 
No. 64.

VIOLET. b. m. 15.2 hands, black points, foaled March 16th, 1868, got by Rysdyk's Hambletonian.

First dam, Sallie Feagles, by Smith's Clay he by Cassius M. Clay.

Second dam, by Hickory.

Third dam of Messenger blood.

\section{No. 65.}

LADY TALMADGE. ch. m. 15.1 hands, strip in face, white off hind ankle, foaled

Star.

got by American

Dam, by Liberty. .

No. 66.

MARY SANDFORD, b. m. 15 hands, white near heels, foaled got by Plunket horse, (half brother to Goldsmith's Maid).

Dam, Lady Sandford, (dam of Jay Gould, formerly Judge Brigham), by American Star.

Plunket Horse, got by Alexander's Abdallah.

No. 67.

FANNY HARRIS. ch. m. 15.2 hands, small star in forehead, foaled got by American Star.

Dam, by Black Lock. 


\section{No. 68.}

VARINA. ch.m. star and strip in face, white off hind foot, (Dam of
Idaho, by foaled 1854, (bred by Col. Wade Hampton, So. Kentucky.) Carolina), got by Hero, son of Bertrand, Jr., out of imported Mania.

First dam, by Sovereign.

Second dam, imported Helen, by imported Priam.

Third dam, Malibran, by Rubens.

Fourth dam, Bolter's dam, by Trumpator.

Fifth dam, by Highflyer.

Sixth dam, by Otho.

Seventh dam, by Snap.

Fighth dam, by Regulus.

Ninth dam, Wildair's dam, by Steady.

Tenth dam, by Partner.

Eleventh dam, by Greyhound.

Twelfth dam, Chestnut Layton, by Makeless. 


\section{SIX YEAR OLD MARES.}

No. 1.

LADY MOSCOW. r.m. black points, foaled August, 5th, 1866, got by Rysdyk's Hambletonian.

First dam, Roan Marie.

Second dam, by Abdallah.

No. 2.

AMANDA. b. m, 15.1 hands, star and snip, little white on off hind heel, foaled 1866, got by Rysdyk's Hambletonian.

First dam, Lady Cardinal, by Cardinal.

Second dam, by One-Eyed Hunter.

No. 3.

FLORA FEAGLES. b. m. 16 hands, star, black points, foaled 1866, got by Rysdyk's Hambletonian.

Dam, Sallie Feagles, by Smith's Clay.

No. 4.

LADY HILL. b. m. 14 hands, star and snip, little white on hind heels, foaled October 22, 1866, got by Rysdyk's Hambletonian.

Dam, by Harry Clay.

Dam of Harry Clay by imported Bellfounder. 


\section{FIVE YEAR OLD MARES.}

No. 1.

RACOLA. br. m. white hind heels, foaled April 22, 1867, got by Rysdylz's Hambletonian.

Dam, Brown Pacer.

No. 2.

ROSETTA. b. m. little white on hind heels, foaled April 22, 1867, got by Rysdyk's Hambletonian.

First dam, Grey Rose, by Harris' Hambletonian, by Bishop's Hambletonian, by imported Messenger.

Dam of Harris' Hambletonian was a Messenger mare, imported by Israel Munson of Boston.

No. 3.

CALYPSO. b. m. small spot on nose, white hind ankles, white fore heels, white hairs in tail, white spot on near side, foaled July 4, 1867, got by Rysdyk's Hambletonian.

Dam, Kate Smith, by old Abdallah.

No. 4.

JOSEPHINE $b$. m. star, white hind ankles, foaled August 13, 1867, got by Rysdyk's Hambletonian.

Dam, Lucy Almack, by young Almack, by old Almack, by Mambrino, by imported Messenger. 
No. 5.

GAY SPANKER, r. m. black points, foaled .July 9th, 1867, got by Rysdyk's Hambletonian.

First dam, Roan Marie.

Second dam, by Abdallah.

No. 6.

BESS. $b$. $m$ star and snip, white near ankles, brown spot behind the shoulder, foaled August 16, 186i, got by Rysdyk's Hambletonian.

First dam, Jesse Sayre, by Harry Clay.

Second dam, by Liberty.

\section{No. $\%$.}

ZENOBIA. br. m. black points, foaled September 18, 1867, got by Rysdyk's Hambletonian.

First dam, Belle, by Post's Hambletonian, by Rysdyk's Hambletonian.

Second dam, by Ethiopian Prince, by Wildair. Third dam, from Ohio. 


\section{FOUR YEAR OLD MARES.}

No. 1.

ZERLINA. b. m. little white on near hind heel, foaled April

19, 1868, got by Rysdyk's Hambletonian.

First dam, Annie Seeley, by Gen'l Gifford.

Second dam, by old Abdallah.

Third dam, by Tom Thumb.

Fourth dam, Kent mare. (dam of Rysdyk's

Hambletonian).

No. 2.

QUEEN OF MAY. b. m. white near hind ankle, white off hind heel, foaled May 1, 1868, got by Rysdyk's Hambletonian.

First dam, Lady Overton, by Abdallah.

Second dam, by Mt. Holly, by old Messenger.

No. 3.

CARRIE. b. m. white hind ankles, foaled May 17, 1868, got by Woburn.

Dam, Jenny Lewis, by black Bashaw. 
No. 4.

STARBEA M. b. .m star, white on lip, white near fore ankle, white hind ankles, foaled June 12, 1868, got by Rydsyk's Hambletonian.

Dam, Montgomery Maid, by American Star.

No. 5 .

UNDINE. b. m. white near hind ankle, foaled June 26, 1868, got by Rysdyk's Hambletonian.

First dam, Roan Marie.

Second dam, by Abdallah.

No. 6.

br. m. black points, foaled 1868, got by Truesdell's Hambletonian.

First dam, Babbitt Abdallah, by old Abdallah.

Second dam, by Phillips, son of Duroc.

Third dam, by Decatur (thoroughbred) son of Henry, sister to Abdallah Chief. 


\section{THREE YEAR OLD FILLIES.}

No. 1.

ADA DUR0C. b. f. white hind ankles, foaled May 15, 1869, got by Messenger Duroc.

Dam, Montgomery Maid, by American Star.

No. 2.

ZEPHYR. b. f. black points, foaled May 15, 1869, got by Idol.

- First dam Miss Wansor, by Sir Archy.

Second dam, by Abdallah.

Third dam, by Westchester.

No. 3.

IDA WEBSTER. b. f. star, white hind heels, foaled May 24, 1869, got by Rysdyk, he by.Rysdyk's Hambletonian.

First dam, Sharpless Abdallah, by Abdallah. Second dam, by Hunter, (sire of Flora Temple and DeLancy mare, called Mist)

No. 4.

SU NBEA M. b. f. white near hind heel, foaled June 20, 1869, got by Idol.

Dam, Lady Packer, by Witherell Messenger, by Winthrop Messenger, by imported Messenger.

No. Б。.

BOULOT'TI. b. f. star and snip, white hind ankles. foaled August 17, 1869, got by Idol.

First dam, Lady Mead, by American Star.

- Second dam, by Saltram. 


\section{TWO YEAR OLD FILLIES.}

\section{No. 1.}

ch. f small star, foaled April 18th, 1870, got by War Eagle.

First dam, Lady Hawkins by old Abdallah.

Second dam, Emily, by Commodore, he by Mambrino.

No. 2.

b. f. white off hind ankle, foaled April 23, 1870 , got by Pierson.

First dam, Bonnie Lassie, by Rysdyk's Hambletonian.

Second dam, by Fox Hunter.

\section{No. 3.}

b. f. star and snip, white hind ankles, foaled May 1, 1870, got by Idol.

First dam, Satinet, by Abdallah Chief, (Roe's.)

Second dam, by Whistle Jack.

Third dam, by the Bertholf Horse.

Fourth dam, by old Duroc.

Abdallah Chief (Roe's), by Abdallah.

First dam, by Phillips, son of Duroc.

Second dam, by Decatur, (thoroughbred) son of Henry.

Whistle Jacket, by Mambrino.

Dam, by American Eclipse.

Bertholf Horse, by imported Messenger. 
Yo. 4.

b. f. white hind ankles, white near fore heel, foaled May 6, 1870, got by Rysdyk's Hambletonian.

First dam, Lucy Almack, by Young Almack, by Old Almack, by Mambrino, by imported Messenger.

No. 5.

LADY BELMONT. br. f. strip in face, white near ankles, foaled May 7, 18\%0, got by Rysdyk's Hambletonian.

First dam, Kate (Bruno's Dam], by Bellaire, the sire of Red Bird.

Second dam, a pacing mare of Comic blood.

No. 6.

br. f. white off hind heel, foaled May 26, 1870, got by Messenger Duroc.

First dam, Mildred, by Rysdyk's Hambletonian.

Second dam, Linda (the old trotting mare.)

No. $\%$.

b. f. star, spot on nose, black points, foaled June 9, 1870, got by Pierson.

First dam, Amanda, by Rysdyk's Hambletonian.

Second dam, Lady Cardinal, by Cardinal. Third dam, by One Eyed Hunter. 
No. 8.

b. f. black points, foaled June 10, 1870, got by Rysdyk's Hambletonian.

First dam, Jennie Lewis, by Black Bashaw.

Nọ. 9.

br. f. star and snip, white hind hecls, foaled June 21, 1870, got by Knickerbocker.

First dam, Nellie Sayres.

\section{No. 10.}

blk. f. white heels, foaled July 26, 1870, got by Messenger Duroc.

First dam, Mattie Bull.

Second dam, by Ohio Eclipse.

Third dam, by Post Boy.

Yo. 11.

br. f. white off fore ankle, white off hind heel. foaled August 3d, 1870, got by Waverly. First dam, Lady Farr, by old Abdallah. 


\section{YEARLING FILLIES.}

No. 1.

b. f. star in forehead, spot on nose, white hind ankles, fonled April 11, 1871, got by Abdallah Star.

First dam, Lady Bryant, by Brignoli, he by old Mambrino Chief.

Second dam, by Cracker, he by Boston the sire of Lexington.

No. 2

b. f. star and snip, white hind heels, foaled April 15, 1871, got by Rysdyk's Hambletonian. First dam, Lady Fallis, by American Star. Second dam, by L I. Black Hawk.

No. 3.

b. f star and snip, white hind ankles, foaled April 19, 1871, got by Pearsall, son of Jupiter. First dam, Lady Irwin, by Rysdyk's Hambletonian.

Second dam, by Roe's Abdallah Chief. 
No. 4.

ch. f. strip in face, white near hind ankle, white off hind heel, foaled May 11, 1871, got by Abdallah Star.

First dam, Mistress Henyon, by Roe's Abdallah/Chief.

Second dam, by Sir Henry.

No. อั.

ch. f. star, white hind ankles, foaled May 12th, 1871, got by Messenger Duroc.

First dam, Lady Overton, by Abdallab.

Second dam, by Mt. Holly, by old Messenger.

No. 6.

ch. f. strip in face, white hind legs, foaled May 16, 1871, got by Messenger Duroc.

First dam, Sallie Feagles, by Smith's Clay, by Cassius M. Clay, Jr.

Second dam, by Hickory, by Mambrino, by imported Messenger.

Third dam of Messenger blood. 


\section{No. 7.}

ch. f. atrip in face, white near fore leg, white hind legs, foaled May 22, 1871, got by Messenger Duroc.

Dam, Stella.

No. 8.

b. f. star and snip, white hind ankles, foaled May 25. 1871, got by Idol.

First dam, Lady Durland, by American Star. Second dam, by Nigger Lance, he by old Lance.

No. 9.

b. f. star and snip, black points, foaled May 25, 1871, got by Messenger Duroc.

First dam, 'Trustus Webb, by American Star. Second dam, by Trustee.

No. 10.

b. f. white hind heels, foaled Nily 26, 1871, got by Messenger Duroc.

First dam, Lady Nodine, by Rysdyk's Hambletonian.

Second dam, Montgomery Maid, by American Star. 
No. 11.

b. f. white face, white fore ankles, white near hind heel, foaled May 28, 1871, got by Messenger Duroc.

First dam, Lady Cardinal, by Cardinal.

Second dam, by imported Bellfounder.

\section{No. 12.}

ch f.white face, foaled May 29, 1871, got by Abdallah Star.

First dam, Ledger Girl, by Rysdyk's Hambletonian.

Second dam, by son of Barney Henry.

\section{No. 13.}

ch. f. white hind heels, foaled June 1, 1871, got by Messenger Duroc.

First dam, Mary Hulse, by American Star.

Second dam, of Messenger blood.

\section{No. 14 .}

b. f. star, white near fore ankle, white hind ankies, foaled July 6, 1871, got by Idol.

First dam, Belle, by Post's Hambletonian.

Second dam, by Ethiopian Prince, by Wildair.

Third dam from Ohio, 
No. 15.

ch. f. star, white hind ankles, foaled July 13, 1871, got by Messenger Duroc.

First dam, Fanny Fiske, by Plough Boy, by L. I. Black Hawk.

Second dam, by Old Almack, by Mambrino.

No. 16.

b. f. white off hind heel, foaled July 15, 1871, got by Messenger Duroc.

First dam, Kitty Mills, by American Star.

Second dam, by Wildair.

No. $1 \%$.

blk. f. star, 'white hind ankles, foaled July 22, 1871, got by Messenger Duroc.

First dam, Virgo, by Roe's Abdallah Chief.

Second dam, by Whistle Jacket.

Third dam, by the Bertholf horse.

Fourth dam, by old Duroc. 


\section{No. 18 .}

b. f. white near hind ankle, white off hind heel, foaled July 24, 1871, got by Conductor. First dam, Lady Talmadge, by American Star. Second dam, by Liberty.

\section{No. 19.}

ch. f. strip in face, white ankles, foaled August 4, 1871, got by Idol.

First dam, Lady Mead by American Star. Second dam, by Saltram. 


\section{N O T I C E.}

\section{I am now ready to offer a portion of my Stock at Private Sale during the year.}

\section{CHARLES BACKMAN.}




\section{3.}

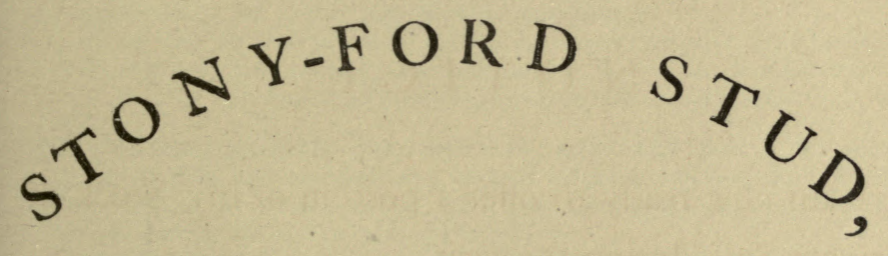

ORANGE COUNTY,

NEW York,

CHARLES BACKMAN.

NEW YORK:

Henky Spear, Printer and Stationer, 82 Wall Street. 


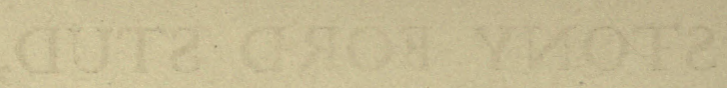




\section{STONY FORD STUD,}

\section{3 .}

The Followixg Stallions are offered to serve a limited number of Marks EACH, in addition to my own, at OxE HCNDlen Dollaks (in advance) for the season, which will commence on the lst of A pril and close on the lst of Octuber.

ABDALIAH STAR.-Chestnut stallion. 16 hands, foaled June 12, 1863. got by American Star, Jr., tirst dam C'urry Abdallah, by Abdallah, son of Mambrino; second dam by imp. Bellfounder; third dam by Royalist; fourth dam by Hardware, son of imp. Messenger.

American Star, Ir., by Seeley's American Star, son of Stockholm's American Star, by Duroc, dam by Friday, son of imp. Trustec.

IDOL,-Bay stallion 15 hands 23/2 inches, foaled June 9, 1864. got by Rysdyk's Hambletonian : first dam Hattie Wood, by Harry Clay; second dam Grandmother, by Terror. son of American Eclipse.

Harry Claj, by Neavé's Cassius MI. Clay. Jr., son of Cassius M. Clay, by Henry Clay, son of Andrew Jackson, by Young Bashaw ; dam by imp. Bellfounder.

N. B. - The above stallions are linited to forty mares each, exclusive of $\mathrm{my}$ own.

MESSENGER DUROC.-Bay stallion, 16 hands, foaled June 3, 1865, got by Kysdyh's Hambletonian; first dam Satinet, by Roe's Abdallah Chief; second dam Catbird. by Whistle Jacket; third dam Lyon mare, by Bertholf horse ; fourth dam by Duroc, son of imp. Diomed.

Hambletonian (Rysdyk's) by Abdallah; first dam Charles Kent mare, by imp. Bellfounder: second dam One Eye. by Bishop's Hambletonian; third dam Silvertail, by imp. Messenger.

Abdallah, by Mambrino, son of imp. Messenger. dam Amazonia, by son of imp. Messenger.

Hambletonian (Bishop's). by imp. Messenger ; first dam Pheasant, by imp. SLark ; second dam by imp. Medley.

Abdallah Chief (Roe's), by Abdallah, son of Mambrino : first dam by Phillips, son of Duroc; second dam by Decatur, (thoroughbred.) son of Henry.

Whistle Jacket, by Mambrino, son of imp. Messenger; first dam by American Eclipse; second dam by Bertholf Horse, son of imp. Messenger.

N. B.-Messenger Duroc's limit for season of 1873 is full, and he is booked for twelve mares for 1874 at TWO HUNDRED DOLLARS the season; Applications from patrons of 1873 , for services for season of 1874 , will be received until January 1, 1874, and given precedence orer new applicants, which will be filed in order of date.

W A VERLEY.-Brown ftallion 15 hands 336 inches, foaled October 9, 1866 got by Rjsdyk's Hambletonian; first dam Susie Roberts, by New York Black Hawk; second dam the well known maro of Messenger descent, owned and driven by Captain Roberts, of New York.

N. Y. Black Hawk by L. I. Black Hawk, son of Andrew Jackson, by Young Bashaw ; first dam sister to Jack Cade, by Lcopold (Arabian) : second dam by Bishop's Hambletonian, son of imp. Messenger.

BOLTON.-Bay stallion, 16 hands $3 / 2$ in:h. foaled June 13. 1867. got by Rysdyk's Hambletoniau, dam Montgomery Maid, by Seeley's American Star.

ELECTION EER.-Bay stallion, 15 hands 2 inches, foaled May 2. 1868, got by Rysdyk's Hambletonian; first dam Green Mountain Maid (Prospero's dam) by Harry Clay, son of Neave's Cassius M. Clay, Jr.

The last three stallions will be limiced to twenty-five mares each, exclusive of my own.

Srowr Fond, Orange County, January 1, 1873.

CHARLES BACKMAN. 



\section{StONy Ford Stud.}

\section{STALLIONS.}

ABDALLAH ch. s. 16 hands, small star, white off hind STAR. ankle, foaled June 12, 1863 ; got by American Star, Jr.

First dam, Curry Abdallah, by Abdallah.

Second dam, by imported Bellfounder.

Third dam, by Rosalist.

Fourth dam, by Hardware, son of imp.

Messenger.

American Star, Jr, by Seely's American Star;

Dam by Friday, son of imp. Trustee.

American Star (Seely's), by Stockholm's

American Star, son' of Durnc.

First dam, Sally Slouch, by Sir Henry.

Second dam, by imp. Messenger.

Friday, by imp. Trustee.

First dam, Saluda, by Timoleon.

Second dam, by imp. Sir Harry.

Third dam, Highland Mary, sister to Sir

Archy, by imp. Diomed.

Trustee (imp.), by ('atton.

First dam, Emma, by Whisker.

Second dam, Gibside Fairy, by Hermes.

Third dam, Vicissitude, by Pipator.

Fourth dam, Beatrice, by Sir Peter.

Fifth dam, Pyrrha, by Matchem.

Sixth dam, Duchess, by Whitenose.

Seventh dam, Miss Slamerkin, by Young 'True

Blue.

Eighth dam, by Lord Oxford's Dun Arabian.

Ninth dam, D'Arcy's hlack legged Royal

Mare.

Abdallah, by Mainbrino, son of imp. Messenger

Dam Amazonia, by son of imp. Messenger. 
ID0L. b. s., $15.2 \frac{1}{2}$ hands, white near fore heel, white hind ankies, foaled June 9, 1864 ; got by Rysdyk's Hambletonian.

First dam, Inattie Wood, by Harry Clar.

Second dam, Grandmother, by Terror.

Hambletonian, (Rysdyk's) by Abdallah.

Eirst dam, Charles Kent Mare, by imp.

Bellfounder.

Sccond dam, One Eye, by Bishop's

- Hambletonian.

Thiru dan, Silvertail, by imp. Messenger. Abdallah, by Manibrino, son of imp Messenger.

Dam, Imazonia, by son of imp. Messenger. Hambletonian, (Bishop's) by imp Messenger,

- First dam, I'heasant, by imp. Shark.

Sucond dam, by imp. Medley.

Harry Clay, by Neave's Cassius M. Clay, Jr.

Dam, by imp. Bellfounder.

Cassius M. Clay, Jr., (Neave's, by Cassius M.

Clay, son of Henry Clay, by Andrew Jackson, son of Young Bashaw.

First dam, by Engineer 2d, (sire of Lady Suffolk).

Engineer 2d, by Engineer, son of imp.

Messenger.

First dam. by Plato.

Second dam, by Rainbow.

Platn, by imp. Messenger.

Fijst dam, Pheasant, by imp. Shark.

Second dim, by imp Nedley.

Rainbow, by imp. Wildair.

First dam, by Gen. Heard's True Britton.

Second dan, an imp. mare, by Duke, of Bolton's, Starling.

Terror, by American Eclipse.

First dam, Lady Lightfoot, by Sir Archy.

Second dam, Black Maria, by imp. Shark. 
Third dam, by imp. Clockfast. Fourth dam, Burwell's Maria, by Regulus. Fifth dam, Camilla, by imp. Fearnaught. Sixth dam, Calista, by Forrester. American Eclipse, by Duroc, son of imp Diomed.

Dam, Miller's Damsel, by imp. Messenger. Sir Archy, by imp. Diomed.

Dam, Castianira, by Rockingham.

PIERSON. b. s. 16 hands, white hind heels, foaled May 1864 ; got by Rysdyk's Haunbletonian.

First dam, hy McChesney's Cassius M. Clay, Jr.

Second Dam, by Black Bird.

Cassius M Clay, Jr. (McChesney's), by Cassius M. Clay, son of Henry Clay, by Andrew Jackson, son of Young Bashaw.

Dam, Lady Free Love, by Abdallah, son ot Mambrino. 
MESSENGER b. s., 16 hands, white hind ankles, foaled DUR0C. June 3, 1865, got by Rysdyk's Hambletonian.

First dam, Satinet, foaled 18.54, by Roe's Abdallith Chief.

Second dam, Catbird, foaled 1834, by Whistle Jacket.

Third dam, Lyon Mare, by Bertholf horse.

Funrth dam, by Duroc, son of imp. Diomed.

Hambletonian (Rysdyk's), by Abdallah.

First dam, Charles Kent Mare, by imp. Bellfounder.

Second dam, One Eye, by Bishop's Hambletonian.

Third dam, Silvertail, by imp. Messenger, Abdallah, by Mambrino, son of imp. Messenger. Dam, Amazonia, by son of imp. Mesaenger. Hambletonian (Bishop's), by imp. Messenger.

First dam, Pheasant, by imp. Shark.

Second dam, by imp. Medley.

Abdallah Chief (Roe's), by Abdallah, son of Mambrino.

First dam, by Philips, son of Duroc.

Second dam, by Decatur, thoroughbred son of Henry.

Whistle Jacket, by Mambrino, son of imp.

Messengtr.

First dam, by Amcrican Eclipse.

Second dam, by Bertholf Horse.

Bertholf Horse, by imp. Messenger.

Dam, by Duruc. 
STONY-FORD. br. s., 152 hands, little white on near hind heel, foaled May 27, 1866; got by Rysdyk's Hambletonian.

First dam, Annie Seely, by Gen. Gifford, son of Gifford Morgan, by Woodbury Morgan.

Second dam, Seely Abdallah, by Abdallah, son of Mambrino.

Third Dam, Belle, by Webber's Tom Thumb. Fourth dam, Charles Kent Mare, (dam of Rysdyk's Hambletonian,) by imp. Bellfounder.

Fifth dam, One Eye, by Bishop's

Hambletonian.

Sixth dam, Silvertail, by imp. Messenger 
WAVERLEY. br. s., 15.3 hands, star, white hind ankles, white off fore heel, foaled October 9, 1866; got by Rysdyk's Hambletonian.

First dam, Susie Roberts, by N. Y. Black Hawk.

Second dam, the well known mare of Messenger descent, owned and driven by Captain Roberts, of New York.

Hambletonian (Rysdyk's), by Abdallah.

First dam, Charles Kent mare, by imp. Bellfounder.

Second dam, One Eye, by Bishop's Hambletonian.

Third dam, Silvertail, by imp. Messenger. Adballah, by Mambrino, son of imp. Messenger.

Dam, Amazonia, by son of imp, Messenger. Hambletonian (Bishop's), by imp. Messenger.

First dam, Phcasant, by imp. Shark.

Second dam, by imp. Medley.

Black Hawk, (New York.) by L.I. Black Hawk. First dam, sister to Jack Cade, by Leopold, (Arabian).

Second dam by Bishop's Hambletonian, son of imp. Messenger.

Black Hawk, (Long Island,) by Andrew Jackson, son of Young Bashaw, by Grand Bashaw.

Dam, Sally Miller, by Mambrino, son of imp. Messenger. 


\section{SIX YEARS OLD STALLIONS.}

\section{No. 1.}

VICTOR b. s., white hind heels, fualed April 19, 1867; von BISMARCK. got by Rysdyk's Hambletonian.

Full Brother to First dam, Hattic IVood, by Harry Clay.
Idol and Gazelle.

Second dam, Grandinother, by Terror.

Hambletonian (Rysdyk's), by Abdallah.

First dam, Charles Kent mare, by imp. Bellfounder.

Second dam, One Eye, by Bishop's Hambletonian.

Third dam, Silvertail, by imp. Messenger. Abdallah, hy Mambrino, son of imp. Messenger.

Dam, Amazonia, by son of imp. Messenger. Hambletonian (Bishop's), by imp. Messenger.

First dam. Pheasant, by imp. Shark.

Second dam, by imp. Medley.

Henry Clay, by Neave's Cassius M. Clay, Jr.

Dam, by imp. Bellfounder.

Cassius M. Clay, Jr. (Neave's), by Cassius M Clay, son of Henry Clay, by Andrew Jackson son of Young Bashaw.

First dam, by Chancellor, son of Mambrino.

Second dam, by Engineer 2d, son of Engineer, by imp. Messenger.

Terror, by American Eclipse.

First dam, Lady Lightfoot, by Sir Archy.

Second dam, Black Maria, by imp. Shark.

Third darn, by imp. Clockfast.

Fourth dam, Burwell's Maria, by Regulus.

Fifth dam, Camilla, by imp. Fearnaught.

Sixth dam, Calista, by Forrester.

American Eclipse, by Duroc son of imp Diomed.

Dam, Miller's Damsel, by imp. Messenger.

Sir Archy, by imp. Diomed.

Dam, Castianira, by Rockingham. 
No. 2.

AITCHESON. b. s., star, white near hind ankle, foaled June

13, 1867; got by Rysdyk's Hambletonian.

First dam, lady Van Buren, by Red lBird.

Second dam, by Young Rellfounder, son of imp. Bellfounder.

Hambletonian (Rysdyk's), by Abdallah.

First dam, Charles Kent Mare, by imp. Bellfounder.

Second dam, One Eye, by Bishop's Hambletonian.

Third dam, Silvertail, by imp, Messenger. Abdallah, by Mambrino, son of imp. Messenger Dam, Amazonia, by son of imp. Messenger. Hanbletonian (Bishop s), by imp. Messenger.

First dam, Pheasant, by imp. Shark.

Second dam, by imp. Medley.

\section{No. 3.}

BOLTON. b. h., 16 hands $\frac{1}{2}$ inch, white oft hind heel, foaled June 13, 1867 ; got by Rysdyk's Hambletonian Dam, Montgumery Maid, by Secly's American Star.

Hambletonian (Rysdyk's), by Abdallah.

First dam, Charles Kent mare, by imp. Bellfounder.

Second dam, "One Eye, by Bishop"s Hambletonian.

Third dam, Silvertail, by imp. Messenger. Abd:llah, by Mambrino, son of imp. Messenger. Dam, Amazonia, by son of imp. Messenger. 
Hambletonian (Bishop's), by imp. Messcnger.

First dam, Pheasant, by imp. Shark.

Second dam, by imp. Medley.

American Star (Seely's), by Stockholm's

American Star, son of Duroc.

First dam, Sally Slouch, by Sir Henry.

Second dam, by imp. Messenger.

Sir Henry, by Sir Archy, son of imp. Diomed

First dam, by imp. Diomed.

Second dam, Bellona, by Bel-Air.

Third dam, Indian Queen, by Pilgrim.

Fourth dam, by imp. Janus.

Fifth dam, by imp. Jolly Roger.

Sixtb dam, by imp. Valiant.

EXCHANGE. g. s., star and snip, white hind ankles, foaled July 4, $1 \$ 567$; got by Rysuyls's Hambletonian.

Dam, Lady Swazey, by Harris' Hambletonian Hambletonian (Harris'), by Bishop's

Hambletonian.

Dam, the mare owned by Israel Munson, of Boston, and represented to be by imp. Messenger.

Hambletonian (Bishop's), by imp. Messenger.

First dam, Pheasant, by imp. Shark,

Second dam, by imp. Medley. 


\section{No. 5.}

KENILWORTH. br. s., black points, foaled Sept. 10, 1867 ;

Brother to Waverley. got by Rysdyk's Hambletonian

First dam, Susie Roberts, by N. Y. Black Hawk.

Second dam, the well-known mare of Messenger descent, owned and driven by Captain Roberts, of New York.

Black Hawk (New York), by L. I. Black Hawk, First dam, sister to Jack Cade, by Leopold, (Arabian.)

'Secorid dam, by Bishop's Hambletonian, son of imp. Messenger.

Black Hawk (Long Island), by Andrew Tuckson, sen of Young Bashaw, by Grand Bashaw.

Dam, Sally Miller, by Mambrino, son of imp. Messenger. 


\section{FIVE YEARS OLD STALLIONS.}

No. 1.

TWILIGHT. b. s., white hind heels, foaled April 20, 1868 ; got by Rysdyk's Hambletonian.

First dam, Mary Hulse, by Scely's American Star.

Second dam, of Messenger descent. American Star (Seely's). by Stockholm's American Star, son of Duroc.

First Dam, Sally Slouch, by Sir Henry.

Second dam, by imp. Messenger.

\section{No. 2.}

ELECTIONEER. b. s., white hind heels, foaled May 2, 1868, got by Rysdyk's Hambletonian.

First dam, Green Mountain Maid, by Harry Clay.

Hambletonian (Rysdyk's), by Abdallah.

First dam, Charles Kent mare, by imp. Bellfounder.

Second dam, One Eye, by Bishop's Hambletonian.

Third dam, Silvertuil, by imp. Messenger. Abdallah, by Mambrino, son of imp. Messenger.

Dam, Amazonia, by son of imp. Messenger. Hambletonian (lishop's), by imp. Messenger.

First dam, Pheasant, by imp. Shark.

Second dam, by imp. Medley.

Harry Clay, by Neave's Cassius M. Clay, Jr.

Dam by imp. Bellfcunder. 
Cassius M. Clay, Jr. (Neave's), by Cassius M. Clay, son of Henry Clay, by Andrew Jackson.

First dam, by Chancellor, son of Mambrino.

Second dam, by Engireer 2d, son of Engineer. Engineer 2d, by Engineer, son of imported Messenger.

First dam, by Plato, son of imp Messenger. Second dam, by Rainbow. Plato, by imp. Messenger.

First dam, Pheasant, by imp. Shark.

Second dam, by imp. Medley.

Rainbow, by imp. Wildair.

First dam, by Gen. Heard's True Britton.

Second dam, by Duke of Bolton's Starling.

No. 3.

TROUBLE. b, s., white hind ankles, foaled May 22, 1868; got by Rysdyk's Hambletonian.

First dam, Lady Hopkins, by a son of Hill's Vermont Black Hawk.

Second dam, by Harris' Hambletonian. Son of Hill's Vermont Black Hawk, by Hill's Vermont Black Hawk, son of Sherman Murgan.

Dam, by Harris' Hambletonian.

Hambletoniau (Harris,) by Bishop's

Hambletonian, son of imp. Messenger.

Dam, the mare owned by Israel Munson, of Boston, and represented to be by imp. Messenger. 
No. 4.

DIRECTOR. b. s., small star, white hind ankles, foaled June 9, 1868; got by Rysdyk's Hambletonian.

First dam, Lady Bellfounder, by Webber's Tom Thumb.

Second Dam, by Crabtree Bellfounder.

Third dam, by imp. Bellfounder.

\section{No. 5.}

BRINEY, b. s, white hind heels, white near foot, foaled 1868; got by Mambrino Pilot.

Fir:t dam, Lady Dexter, by Rysdyk's Hambletonian,

Second dam (Dexter's dam,) by American Star.

Mambrino Pilot, by Mambrino Chief, son of Mambrino Paymaster. Dam, by Alexander's Pilot, Jr. 


\section{THREE YEARS OLD COLTS.}

No. 1.

STII.LSON. ch. c., white hind ankles, foaled May 21, 1870 ; got by Messenger Durøe, son of Rysdyk's Hambletonian

Dam, Grey Rose, by Harris' Hambletonian. Hambletonian (Harris',) by Bishop's Hambletonian.

Dam, the mare owned by Israel Munson, of Boston, and represented to be by imp. Messenger.

Hambletonian (Bishop's), by imp. Messenger.

First dam, Pheasant, by imp. Shark.

Second dam, by imp. Medley.

No. 2.

AUGUST BELMONT. b. c., white hind heels, foaled May

22, 1870 ; got by Rysdyk's Hambletonian.

First dam, Miss Wansor, by Jacksou's Sir Archy, by Bay State, son of Green Mountain Morgan, by Gifford Morgan.

Second dam, by Westchester, son of L. I. Black Hawk.

Third dam, by Abdallah.

Fourth dam, by Engineer 2d, (sire of Lady Suffolk.) 
Black Hawk (Long Island), by Andrew Jackson, son of Young Bashaw, by Grand Bashaw. Dam, Sallie Miller, by Mambrino.

Abdallah, by Mambrino, son of imp. Messenger. Dam, Amazonia, by son of imp. Messenger. Engineer 2d, by Engineer, son of imp. Messenger.

First dam, by Plato.

Second dam, by Rainbow.

Plato, by imp. Messenger.

First dam, Pheasant, by imp. Shark.

Second dam, by imp. Medley.

Rainbow, by imp. Wildair.

First dam, by Gen. Heard's True Britton.

Second dam, an imported mare, by Duke of Bolton's Starling.

LEGAL. b. c., star, white off hind heel, white near hind ankle, white near fore leg, foaled July $3 d, 1870$, got by Rysdyk's Hambletonian.

First dam, Fannie Fiske, by Young Almack. Second dam, by Jonas Flower's horse Sportsman,

Almack. (Young) by Almack, son of Mambrino.

Dam, by Diamond, son of American Eclipse. Sportsman. (Jonas Flowers) by Chancellor, son of Mambrino.

Dam, by Sir Henry, son of Sir Archy. 
No. 4.

ELLW00D. b. c., star, white hind ankles, foaled July 14th, 1870, got by Messenger Duroc, son of Rysdyk's Hambletonian.

First dam, Virgo, by Roe's Abdallah Chief. Second dam, Catbird, by Whistle Jacket.

Third dam, by Bertholf Horse.

Fourth dam, by Duroc, son of imp. Diomed. Abdallah Chief, (Roe's) by Abdallah, son of Mambrino.

First dam, by Philips, son of Duroc.

Second dam, by Decatur, thoroughbred son of Henry.

Whistle Jack, by Mambrino, son of imp. Messenger.

Dam, by American Eclipse.

Bertholf Horse, by imp. Messenger.

Dam, by Duroc.

No. 5 .

RIDGEWOOD. b, c., star and snip, white near hind ankle, foaled 1870; got by Rysdyk's Hambletonian.

First dam, Hoe Mare, by Wilson's Sir Henry.

Second dam, by American Eclipse.

Third dam, by Redbird, son of Bishop's Hambletonian. 


\section{TWO YEARS OLD COLTS.}

No. 1.

ORPHAN. ch. c., strip in face, white near hind ankle, foaled May 5th, 1871; got by Abdallah Star, son of American Star, Jr., by Seely's American Star.

First dam, Lady Rockafellow, by Seely's American Star.

Second dam, by Bay Richmond.

Third dam, by Gilbert Oliver's Jackson, by son of imp. Messenger.

American Star, (Seely's) by Stockholm's American Star.

First dam; Sally Slouch, by Sir Henry.

Second dam, by imp Messenger,

Bay Richmond, by Toby, son of imp. Brown Highlander.

Dam, Lady Pluck, by Mambrino, son of imp. Messenger.

No. '2.

QUARTETTE. ch. c., white hind aukles, foaled May 15th, 1871; got by Idol, son of Rysdyk's . Hambletonian.

First dam, Emma Hoyt, by Seely's American Star.

Second dam, by Ohio Eclipse, son of American Eclipse.

Third dum, by Messenger descent.

-American Star (Seely's), by Stockholm's American Star.

First dam, Sally Sloueh, by Sir Henry. Second dam, by imp. Messenger. 
No. 3.

LEGION. blk. c., white hind heels, foaled May 30th, 1871; got by Messenger Duroc, son of Rysdyk's Hambletonian.

First dam, Sharpless Abdallah, by Abdallah. Second dam, by One Eyed Kentucky Hunter. Abdallah; by Mambrino, son of imp. Messenger. Dam, Amazonia, by son of imp. Messenger.

No. 4.

CARL S. BURR. b. c., star, white near fore heel, white near hind heel, small white spot on off hind heel ; foaled May 31st, 1871; got by Rysdyk's Hambletonian.

Dam, Kate, dam of Bruno.

ROCKET. ch. c, white hind ankles ; foaled June 17, 1871 ; got by Messenger. Duroc, son of Rysdyk's Hambletonian.

First dam, Susie Roberts, by N. Y. Black Hawk.

Second dam, the well known mare of

Messenger descent, owned and driven by Capt. Roberts, of New York.

Black Hawk (New York), by L. I. Black Hawk.

First dam, sister to Jack Cade, by Leopold, (Arabian.)

Second dam, by Bishop's Hambletonian, son of imp. Messenger. 
No. 6.

PROSPECT. blk c., star, white hind ankles, foaled June 24th, 1871; got by Messenger Duroc, son of Rysdyk's Hambletonian.

Dam, Montgomery Maid, by Seely's American Star.

American Star (Secly's), by Stockholm's American Star.

First dam, Sally Slouch, by Sir Henry.

Second dam, by imported Messenger.

No. 7.

CORSAIR. b. c., star, white spot on off hind heel, foaled July 18th, 1871; got by Rysdyk's Hambletonian. First dam, Kate Seely, by Walden Messenger. Second dam, by Mambrino, son of imp. Messenger.

No. 8.

LIGHTEN. b. c., white hind ankles, foaled July 20 th, 1871; got by Messenger Duroc, son of Rysdyk $\mathrm{s}$ Hambletonian.

First dam, Babbitt Abdallah (sister to Roe's Abdallah Chief), by Abdallah. Second dam, by Philips, son of Duroc. Third dam, by Decatur, thoroughbred son of Henry.

No. 9.

THACKERY. b. c., strip in forehead, white off hind ankle, foaled August 5, 1871; got by Messenger Duroc, son of Rysdyk's Hambletonian.

Dam. Lady Ellis, by Alexander's Abdallah, (formerly Edsell's Hambletonian.) Abdallah (Alexander's), by Rysdyk's Hambletonian.

First dam, Katy Darling, by Bay Roman. Second dam, by Mambrino, son of Mambrino, by imported Messenger. 


\section{YEARLING COLTS.}

No. 1.

MARENGO. b. c., white hind heels, foaled April 17, 1872; got by Messenger Duroc, son of Rysdyk's Hambletonian.

First dam, Hattie Hogan, by Harry (lay, son Neave's Cassius M. Clay, Jr. Second dam, Nellie Sayres, by Seely's American Star.

Third dam, by Hulse's Hickory.

Fourth dam, by Lattourett's Bellfounder.

No. 2.

b. c, white hind heels, foaled April «9, 1872; got by Messenger Duroc, son of Rysdyk's Hambletonian.

First dam, Waverly Howland, by Seely's American Star.

Second dam, by Roebuck, son of Blackbird.

No. 3.

MANDARIN. b. c.. star white hind heels, foaled April 30, 187.2; got by Idol, son of Rysdyk's Hambletonian. First dam, Trustus Webb, by Seely's American Star.

Second dain, by imp. Trustee. 
No. 4.

PROCTOR. b. c., star white near hind heel, foaled May 3, 1872; got by Abdallah Star, son of American Star, Jr, by Seely's American Star.

First dam, Bonnie Lassie, by Rysdyk's Hambletonian.

Second dam, by Fox Hunter.

No. 5 .

b. c., white off bind heel, white strip on near fore foot, foaled May 14, 1×7: ; got by Idol, son of Ryskyk's Hambletonian.

Dam, by Charles E. Loew (formerly Patcluen Chief.)

No. 6.

b. c., white hind legs, foaled May 17, 1872; got by Imperial, son of Rysdyk's Hambletonian.

First dam, Mary Sanford, by Plunket Horse, son of Alexander's Abdallah.

Second dam, Lady Sanford, by Seely's American Star.

No. 7.

KING WILLIAM. b. c.. white hind ankles, foaled May 19, 1872 ; got by Rysdyk's Hambletonian.

First dam, Mary Hulse, by Seely's American Star.

Second dam, of Messenger descent. 
No. 8.

ch. c., strip in face, white hind legs, foaled May 19, 1872; got by Abdallah Star, son of American Star, Jr., by Seely's American Star.

First dam, Adele, by Rysdyk's Hambletonian. Second dam, Lady Franklin (owned by David Bonner, Esq.)

No. 9.

b. c., white near hind ankle. foaled May 20, 1872 ; got by Rysdyk's Hambletonian.

Dam, Kate, the dam of Bruno.

No. 10.

PICKERING. b. c., suip on nose, white near fore ankle, (Brother to
Socrates.) white hind ankles, foaled May 23, 1872; got by Rysdyk's Hambletonian.

First dam, Lady Fallis, by Seely's American Star.

Second dam, by Long Island Black Hawk.

No. 11.

ANTENOR. br. e., white ankles, foaled May 25,1872 ; got by Messenger Duroc, son of Rysdyk's Hambletonian.

Dam, Lucy Almack, by Young Engineer, son of Engineer 2d, sire of Lady Suffolk. 
No. 12.

MARQUIS. b. c, star, white near hind ankle, foaled May 26,1872 ; got by Idol, son of Rysdyk's Hambletonian.

First dam, Belie of Richmond, by Rysdyk's Hambletonian.

Second dam, old Belle of Richmond, by Bathgate's Grey Norman.

\section{No. 13.}

FERDINAND. b. c., strip in face, white hind ankles, foaled May 31, 1872; got by Idol, son of Rysdyk's Hambletonian.

First dam, Mistress Henyon, by Roe's Abdallah Chief, son of Abdallah.

Second dam, by Sir Henry.

\section{No. 14.}

CONQUEST. ch. c., strip in face, white near fore ankle, white off fore heel, white hind ankles, foaled June 6, 1872; got by Idol, son of Rysdyk's Hambletonian.

First dam, Wells Star, by Seely's American Star.

Second dam, by Bertrand, son of Kentucky Bertiand.

\section{No. 15.}

OVATION. ch. c., star and snip, white fore ankle, white hind legs, foaled June 8, 1872; got by Idol, son of Rysdyk's Hambletonian.

First dam, Lady Orerton, by Abdallah, son of Mámbrino.

Second dam, by Mount Holly, son of imp. Messenger. 
No. 16.

MOHICAN. b. c, white off hind ankle, foaled June 9, 1872 ; got by Rysdyk's Hambletonian.

First dam, Maggie Jones, by Seely's American Star.

Second dam, by Mambrino Chief, son of Mambrino Paymaster, by Mambrino.

No. 17 .

VICTOR DUROC. blk. c., snip on nostril, white near fore ankle, white hind legs, watch eye on near side, white tip on tail, foaled June 2र̂zd, 1872; got by Messenger Duroc, son of Rysdyk's Hambletonian.

First Dam, Lady Dexter, by Rysdyk's Hambletonian.

Second dam, Dexter's dam, by Seely's American Star.

No. 18.

EVANDER. b. c., star and snip, white near lind heel, foaled June 25th, 1872; got by Idol, son of Rysdyk's Hambletonian.

First dam, Alice Van Duzer, by Seely's American Star.

Second dam, by Messenger Duroc, son of Sir Archy Duroc.

Third dam, by Paymaster, son of imp. Brown Highlander. 
No. 19.

PERICLES, b. c., snip, white near hind leg, white off hind heel, foaled June 25th, 18i2; got by Rysdyk's Hambletonian.

Dam, Laảy Talmadge, by Seely's American Star.

No. 20,

FALCON. b. c., black points, foaled July 28th, 1872; got by Messenger Duroc, son of Rysdyk's Hambletonian.

First dam, Sallie Feagles, by Smith's Clay, son of Neave's Cassius M Clay, $J$ r.

Second dam, by Hickory, son of Hickory, by imp. Whip.

No. 21,

SATYR, b. c., black points, foaled August 14, 1872; got by Waverley, son of Rysdyk's Hambletonian.

First dam, Lady Pierson, by Neave's Cassius M. Clay, Jr.

Second dam, by Diamond, son of American Eclipse. 


\section{No. 22.}

RINGOES. b. c., star and snip, white near fore fetlock, white near bind ankle, white off hind heel, foaled 1872; got by Messenger Duroc, son of Rysdyk's Hambletonian,

First dam, by Rockafellow's Cassius M. Clay. $\mathrm{Jr}$., son of Cassius M. Clay,

Secund dam, by Seely's American Star.

Third dam, by Bay Richmond, son of Toby, by imported lirown Highlander.

Fourth dam, by Gilbert Olivers Jackson, by son of imported Messenger.

No. 23.

RICHW OOD. b. c., strip in face, black points, foaled 1872 , got by Rysdyk's Hambletonian.

First dam, Hoe Mare, by Wilson's Sir Henry.

Second dam, by American Eclipse.

Third dam, by Redbird, son of Bishop's Hambletonian. 


\section{EIGHT YEARS OLD GELDING.}

DEAN. b. g, white near fore foot, white near hind ankle, white off hind foot, foaled 1865 ; got by Rysdyk's Hambletonian.

Dam, by Seely's American Star.

\section{SEVEN YEARS OLD GELDING.}

ISLAND BOY. b. g., white off heels, foaled 1866 ; got by Hetzell Hambletonian, son of Rysdyk's Hambletonian.

First dam, Lady Durland, by Seely's American Star.

Second dam, by Nigger Lance, son of Lance, thoroughbred son of American Eclipse. 


\section{FIVE YEARS OLD GELDING.}

SILVER HEEL, b. g., white near hiild heel, foaled May 9, 1868; got by Rysdyk's Hambletonian.

Dam, Rosa Lee, by Harris' Hambletonian. . Hambletonian (Rysdyk's), by Abdallah.

First dam, Charles Kent Mare, by imp. Bellfounder.

Sec:ond dam, One Eye, by Bishop's Hambletonian.

Third dam, Silvertail, by imp. Messenger. Abdallah, by Mambrino, son of imp. Messenger. Dam, Amazonia, by son of imp. Messenger. Hambletonian (Bishop's), by imp. Messenger.

- First dam, Pheasant, by imp. Shark.

Second dam, by imp. Medley, Hambletonian, (Harris') by Bishop's Hambletonian, son of imp Messenger.

Dam, the mare owned by Israel Munson, of Boston, and represented to be by imp. Messenger. 


\section{FOUR YEARS OLD GELDINGS.}

\section{No. 1.}

TRIUMPH. b. g., white hind heels, foaled April 26,1869 , got by Idol, son of Rysdyk's Hambletunian

Dəm, Rosa Lee, by Harris' Hambletonian. Hambletonian, (Harris') by Bishop's

Hambletonian.

Dam, the Messenger Mare, owned by Israel Munson, of Boston

Hambletonian, (Bishop's) by imp. Messenger.

First dam, Pheasant, by imp. Shark.

Second dam, by imp. Medley.

\section{No. 2.}

LUCKNOW. b. g., star and snip, white near hind heel, white off fore heel, foaled May 11, 1869; got by Idol, son of Rysdyk's Hambletonian.

Dam, Grandmother, (grandam of Idol) by Terror, son of American Eclipse.

\section{No. 3.}

PICK WICK. br. g., star and snip; white hind ankles, little white on near fore heel, foaled June 9, 1869; got by Idol, son of Rysdyk's Hambletonian.

First dam, Lady Brown, by Seely's American Star.

Second dam, by Nigger Lance, son of Lance, by American Eclipse. 
No. 4.

GEORGE ch. g, star and snip, white hind heels, white GARDINER. near fore heel, foaled September 9, 1869; got by Messenger Duroc, son of Rysdyk's Hambletonian.

First dam, Hattie Wood, dam of Idol, by Harry Clay.

Second dam, Grandmother, by Terror. Harry Clay, by Neave's Cassius M. Clay, Jr.

Dam, by imported Bellfounder.

Cassius M. Clay, Jr. (Neave's), by Cassius M. Clay, son of Eenry Clay, by Andrew Jackson.

First dam, by Chancellor, son of Mambrino.

Second dam, by Engineer 2d, son of kngineer, by imp. Messenger.

Terror, by American Eclipse.

First dam, Lady Lightfoot, by Sir Archy.

Second dam, Black Maria, by imp. Shark.

Third dam, by imp. Clockfast.

Fourth dam, Burwell's Maria, by Regulus.

Fifth dam, Camilla, by imp. Fearnaught.

Sixth dam, Calista, by Forrester, 


\section{THREE YEARS OLD GELDINGS.}

\section{No. 1.}

GUY. b. g., strip in face, white off fore ankle, white hind ankles, foaled April 24, 1870 ; got by Pierson, son of Rysdyk's Hambletonian.

First dam, Zuleika, by N. Y. Black Hawk, son of L. I. Black Hawk.

Second dam, by Mambrino Paymaster, son of Mambrino, by imp. Messenger.

No. 2.

LEGATE. b. g., white hind ankles, foaled May 5th, 1870 ; got by Pierson, son of Rysdyk's Hambletonian.

First dam, Alice Van Duzer, by Seely's American Star.

Second dam, by Messenger Duroc, son of Sir Archy Duroc.

Third dam, by Paymaster, son of imp. Brown Highlander.

No. 3.

STARTLlNG. blk g., white near fore heel, white hind heels, foaled June 5, 1870; got by Messenger Duroc, son of Rysdyk's Hambletonian.

First dam, Montgomery Maid, by Seely's American Star. 
No. 4.

GLENEIDA. ch. g, star, white hind heels, foaled June 25, 1870 ; got by Messenger Duroc, son of Rysdyk's Hambletonian.

First dam, Lady Nodine, by Rysdyk's Hambletonian.

Second dam, Montgomery Maid, by Seely's A merican Star.

\section{No. 5.}

b. g., white hind heels, foaled July 6, 1870; got by Pierson, son of Kivsdyk's Hambletonian.

Dam, Roan Marie.

\section{TWO YEARS OLD GELDINGS.}

\section{No. 1.}

TASSO. b. g., star and snip, white hind ankles, foaled April 14, 1871; got by Waverly, son of Rysdyk's Hambletonian.

First dam, Zuleika, by N. Y. Black Hawk. son of L. I. Black Hawk.

Second dam, by Mambrino Paymaster, son of Mambrino, by imp. Hessenger. 
No. 2.

MACLURE. blk. $g$, star, white off fore foot, white hind ankles, foaled April 21, 1871; got by Messenger Duroc, son of Rysdyk's Hambletonian.

First dam, Nellie Wilmarth, by Raven, son of Hill's Vermont Black Hawk.

Second dam, Jennie Lind, by Young Andrew Jackson, son of Andrew Jackson.

Third dam, Yolly Sherman, by Bush Messenger, (Palmer's horse) son of imp. Messenger.

\section{No. 3.}

EPICURE. b. g., star in forehead, foaled April 28th, 1871; got by Waverly, son of Rysdyk's Hambletonian. First dam, Lady Pierson, by Neave's Cassius M. Clay, Jr., son of Cassius M. Clay.

Second dam, by Diamond, son of American Eclipse.

Third dam, a running mare, said to be thoroughbred.

No. 4.

GLENDENNING. ch. g., star and snip, white fore ankles, white near hind ankle, foaled May 28th, 1871; got by Messenger Duroc, son of Rysdyk's Hambletonian.

Dam, Glencoe Maid. 
No. 5.

MARATH0N. b. g., white near fore foot, white hind ankles, foaled June 5th, 1871 ; got by Messenger Duroc, son of Rysdyk's Hambletonian.

Dam, Lucy Almack, by Young Engineer, son of Engineer 2d, sire of Lady Suffolk.

No. 6.

DAZZLE. b. g., star, black points, foaled June 25th, 1871; got by Messenger Duroc, son of Rysdyk's Hambletonian.

Dam, Lady Packer, by Witherell Messenger, son of Winthrop Messenger, by imp. Messenger.

CADMUS. b. g., star and snip, white hind heels, foaled July 16th, 1871; got by Abdallah Star, son of A merican Star, Jr., by Seely's American Star. First dam, Alice Van Duzer, by Seely's American Star.

Second dam, by Messenger Duroc, son of Sir Archy Duroc.

Third dam, by Paymaster, son of imp. Brown Highlander. 


\section{BROOD MARES.}

No. 1.

ALICE VAN DUZER. blk. mare, 15 hands, strip in face, white hind inkles, white near fore heel, foaled 1852; got by Seely's American Star.

First dam, by Messenger Duroc.

Second dam, by Paymaster

American Star (Seely's), by Stockholm's

American Star.

First dam, Sally Slouch, by Sir Henry.

Second dam, by imp. Messenger.

Messenger Duroc, by Sir Archy Duroc, son of

Duroe.

Dam, by Messenger, son of imp. Messenger.

Paymaster, by imp. Brown Highlander.

First dam, by imp. Highflyer.

Second dam, by Rainbow, son of imp Wildair.

Third dam, ky True Briton.

No. 2.

LADY BROWN. br. m., 15 hands, star and snip, white (Dam of Gauntlet off hind heel, foaled 1861; got by Seely's wilkins Mlicawber) American Star.

First dam, by Nigger Lance, son of Lance. American Star, (Seely's) by Stockholm's

American Star, son of Duroc.

First dam, Sally Slouch, by Sir Herry.

Second dam, by imp. Messenger.

Lance, by American Eclipse.

Dam, the dam of Ariel, by Financier. 
No. 3.

LADY BELLFOUNDER. ch. m., 15.2 hands, strip in face, "Walling Mare " white near lind leg up to hock, white off hind ankle, foaled $1 \& 56$; got by Webber's Tom Thumb.

First dam, by Crabtree's Bellfounder.

Second dam, by imp. Isellfounder.

No. 4.

SALLIE FEAGLES. br. m., $15.2 \frac{1}{2}$ hands, black points, foaled 1854; got by Smith's Clay, son of Neave's Cassius M. Clay, Jr.

First dam, by Hickory.

Second dam, of Messenger descent.

Cassius M. Clay, Jr. (Neaves), by Cassius M.

Clay,son of Henry Clay, by Andrew Jackson. First dam, by Chancellor, son of Mambrino.

Second dam, by Engineer 2d, son of Engineer, by imp. Messenger.

Hickory, by Hickory, son of imp. Whip.

First dam, by Mambrino, son of imp. Messenger.

Second dam, by imp. Highlander.

No. 5.

LUCY ALMACK. b. m., 15.2 hands, star, white off hind ankle, white near fore heel, foaled 1852 ; got by Young Engineer, son of Engineer «d, sire of Lady Suffolk. 
No. 6.

MAGGIE JONES. $\mathrm{ch}$. m., 15.1 hands, star, little white on near hind heel, foaled 1852 ; got by Seely's American Star.

Dam, by Mambrino C'hief, son of Nambrino Paymaster.

American Star, (Seely's) by Stockholm's Amcrican Star, son of Duroc.

First dam, Sally Slouch, by Sir Henry.

Second dam, by imp. Messenger.

No. $\%$.

ANNIE SEEJY. b.m., $151 \frac{1}{2}$ bands, black points, foaled 1858; got by General Gifford, son of Gifford Morgan, by Woodbury Morgan.

First dam, Seely Abdallab, by Abdallah, son of Mambrino.

Second dam, Belle, by Webber's Tom Thumb. Third dam, Charles Kent Mare, dam of Rysdyk's Hambletonian, by imp. Bellfounder.

Fourth dam, One Eye, by Rishop's Hambletonian.

Fifth dam, Silvertail, by imp. Messenger.

No. 8.

LADY DURLAND. ch. m., 15 hands, star and snip, foaled 1853, got by Seely's American Star.

Dam, by Nigger Lance, son of Lance.

American Star, (Seely's) by Stockholm's

American Star, son of Duroc.

First daw, Sally Slouch, by Sir Henry.

Second dam, by imp. Messenger.

Lance, by American Eclipse

First dam, dam of Ariel, by Financier.

Second dam, Empress, by imp. Baronet. 
No. 9.

MISS WANSOR. b. m., 16.1 hands, black points, foaled 1860 ; got by Jackson's Sir Archy, son of Bay State.

First dam, by Westchester, son of L. I. Black Hawk.

Second dam, by Abdallah.

Third dam, by Engineer 2d, sire of Lady Suffolk.

Black Hawk, (Loug Island) by Andrew Jackson, son of Young Bashaw, by Grand Bashaw.

Dam, Sallie Miller, by Mambrino, son of imp.

Messenger.

Abdallah, by Mambrino, son of imp. Messenger.

Dam, Amazonia, by son of imp. Messenger.

Engincer 2d, by Engineer, son of imp. Messenger.

First dam, by Plato.

Second dam, by Rainbow.

Plato, by imp. Messenger.

First dam, Pheasant, by imp. Shark.

Second dam, by imp. Medley.

Rainbow, byimp. Wildair.

First dam, by General Heard's True Britton, Second dam, an imp. mare, by Duke of Bolton's Starling. 
No. 10.

BELLE OF RICHMOND. gr. $\mathrm{m}, 15,1$ hands, white near hind ankle, foaled 1861; got by Rysdyk's Hambletonian.

Dam, Belle of Richmond, by Bathgate's Grey Norman.

Hambletonian (Rysdyk's), by Abdallah.

First dam, Charles Kent Mare, by imp.

Bellfounder.

Second dam, One Eye, by Bishop's

Hambletonian.

Third dam, Silver Tail, by imp. Messenger.

Abdallah, by Mambrino, son of imp. Messenger.

Dam, Amazonia, by son of imp. Messenger.

Hambletonian (Bishop's), by imp. Messenger.

First dam, Pheasant, by imp. Shark.

Second dam, by imp. Medley.

Norman (Bathgate's), by Morse Horse.

Dam, by Bishop Hambletonian, son of imp.

Messenger.

No. 11.

LADY PACKER. yr. m, $15 . \hat{z}$ hands, black points, foaled 1860; got by Witherell's Messenger, son of Winthrop Messenger, by imp Messenger. 


\section{No. 12.}

MISTRESS HENYON, ch. m., 15.2 hands, star and strip white hind legs, white near fore heel, fouled 1857 ; got by Roe's Abdailah Chief.

Dam, by Sir Henry.

Abdallah Chief (Roe's), by Abdallah, son of Mambrino.

First dam, by Philip's, son of Duroc.

Second dam, by Decatur (thoroughbred), son of Henry.

Sir Henry, by Sir Areliy, son of imp. Diomed.

First dam, by imp. Diomed.

Second dam, Bellona, by Bel-Air, son of imp. Medley.

No. 13.

MILDRED b. $\mathrm{m}, 15$ hands, star, white off hind ankle, foaled 1858; got by Rysdyk's Hambletonian.

Dam, Linda (the old trotting mare), by Abel Horse, son of Smith's Napoleon, by Napoleon.

Hambletonian (Rysdyk's). by Abdallah.

First dam, Charles Kent Nare, by imp.

Bellfounder.

Second dam, One Eye, by Bishop's

Hambletonian.

Third dam, Silvertail, by imp. Messenger. Abdallah, by Mambrino, son of imp. Messenger.

Dam, Amazonia, by son of imp. Messenger. Hambletonian (Bishop's), by imir. Mrssenger.

First dam, Pheasant, by imp. Shark.

Second Jam, by imp. Medley.

Napoleon, by Young Mambrino.

Dam, by American Commander.

Young Mambrino, by Chancellor, son of

Mambrino, by imp. Messenger.

Dam, by Duroc.

American Commander, by ('ommander, son of imp. Messenger.

Dam, by imp. Light Infantry. 
No. 24.

GREEN MOUNTAIN MAID. br. m., 15 hands, star, white (Dam of Prospero) hind ankles, foaled 1862; got by Harry Clay. Harry Clay, by Neave's Cassius M. Clay, Jr. Darn, by imp. Bellfounder.

Cassius M. Clay, Jr. (Neave's), by Cassius M. Clay, son of Henry Clay, by Andrew Jackson.

First dam, by Chancellor, son of Mambrino.

Second dam, by Engineer 2d (sire of Lady Suffolk), by Engineer.

Engineer 2d, by Engineer, son of imp.

Messenger.

First dam, by Plato.

Second dam, by Rainbow.

Plato, by imp. Messenger.

First dam, Pheasant, by imp. Shark.

Secoud dam, by imp. Medley.

Rainbow, by imp. Wildair.

First dam, by Gen. Heard's True Britton.

Second dam, an imp. mare, by Duke of Bolton's Starling.

No. 15.

KITTY MILLS. b. m., 15.1 hands, snip in face, black points, foaled 1856; got by Seeley's American Star.

Dam, by Wildair.

American Star (Seely's), by Stockholm's

American Star, son of Duroc.

First dam, Sally Slouch, by Sir Henry.

Second dam, by imp. Messenger.

Wildair, by Decatur, son of imp. Messenger.

Dam, by Roebuck, son of Bold Partner. 
No. 16.

LADY OVERTON. ch. m., $15.2 \frac{1}{2}$ hands, strip in face, white off hind ankle, foaled 1851; got by Abdallah. Dam, by Mount Holly. Abdallah, by Mambrino, son of imp. Messenger. Dam, Amazonia, by son of imp. Messenger. Mount Holly, by imp. Messenger.

First dam, by Bajazet, son of imp. Tanner.

Second dam, by Bashaw, son of imp. Wildair.

NELLIE WILMARTH. br. in., 15 hands, star, white hind beels ; foaled June, 1855, got by Raven

First dam, Jennie Lind, by young Andrew Jackson.

Second dam, Polly Sherman, by Bush

Messenger (Palmer Horse), son of Winthrop

Messenger, by imp. Messenger.

Raven, by Hill's Vermont Black Hawk, son of Sherman Morgan.

Andrew Jackson (Young), by Andrew Jackson, son of Young Bashaw, by Grand Bashaw.

Dam, Mambrino mare, Great Western. 
No. 18.

FANNIE FISKE. b. m., 15.2 hands, white near hind leg, white fore heel, foaled 1853; got by Young Almack.

Dam, by Jonas Flowers' Sportsman.

Almack (Young), by Almack.

First dam, by Diamond, son of American Eclipse.

Second dam, by Mambrino, son of imp. Messenger.

Almack, by Mambrino, son of imp. Messenger.

Dam, Sophonisba (mate of Amazonia), by a grandson of imp. Baronet.

Sportsman (Jonas Flowers), by Chancellor, son of Mambrino.

Dam, by Sir Henry, son of Sir Arcby.

\section{No. 19.}

VIRG0. blk. m., 15.1 hands, white near hind heel, foaled 1855 ; got by Abdallah Chief (Roes).

First dam, Catbird, foaled 1834, by Whistle Jacket.

Second dam, Lyon Mare, by Bertholf Horse. Third dam, by Duroc, son of imp. Diomed.

Abdallah Chief (Roe's), by Abdallah.

First dam, by Philip's son of Duroc.

Second dam, by Decatur; thoroughbred, son of Henry.

Abdallah, by Mambrino, son of imp. Messenger.

Dam, Amazonia, by son of imp. Messenger.

Whistle Jacket, by Mambrino, son of imp.

Messenger.

First dam, by American Eclipse.

Second dam, by Bertholf Horse.

Bertholf Horse, by imp. Messenger.

Dam, by Duroc. 
No. 20.

MARY HULSE. b. $\mathrm{m}, 15$ hands, white hind ankles, foaled 1860 ; got by Seely's American Star. Dam of Messenger descent. American Star (Seely's), by Stockholm's American Star, son of Duroc. First dam, Sally Slouch, by Sir Henry. Second dam, by imp. Messenger.

FANNIE STAR. b. m., 15.2 hands, black points, foaled 1860 ; got by Seely's A merican Star.

Dam by Abdallah.

American Star (Seely's), by Stockholm's American Star, son of Duroc. First dam, Sally Slouch, by Sir Henry. Second dam, by imp. Messenger. Abdallah, by Mambrino, son of imp. Messenger. Dam, Amazonia, by son of imp. Messenger. 


\section{No. 22.}

TRUSTUS WEBB. b. m., 15.2 hands, black points, foaled 1860 ; got by Seely's American Star.

Dam by imp. Trustee.

American Star (Seely's), by Stockholm's

American Star, son of Duroc.

First dam, Sally Slouch, by Sir Henry.

Second dam, by imp. Messenger.

Trustee (imp.), by Catton.

First dam, Emma, by Whisker.

Second dam, Gibside Fairy, by Hermes.

Third dam, Viscissitude, by Pipator.

Fourth dam, Beatrice, ky Sir Peter.

Fifth dam, Pyrrha, by Matchem

Sixth dam, Dutchess, by Whitenose.

Seventh dam, Miss Slamerkin, by Young

True Blue.

Eighth dam, by Lord Oxford's Dun Arabian.

Ninth dam, D'Arey's black legged Royal

Mare

No. 23.

CURRY ABDALLAH. ch. m., 16 hands, star and snip, Abdallah of Star.) white hind heels, foaled 1847; got by Abdallah. First dam, by imported Bellfounder.

Second dam, by lioyalist.

Third dam, by Hardware, son of imp.

Messenger.

Abdallah, by Mambrino, son of imp. Messenger.

Dam, Amazonia, by son of imp. Messenger. 
No. 24.

SHARPLESS ABDALLAH. br. m., 15.1 hands, white off hind heel, got by Abdallah.

Dam, by One Eyed Kentucky Hunter.

Abdallab, by Mambrino, son of imp. Messenger.

Dam, Amazunia, by son of imp. Mossenger.

No. 25.

LADY PIERS0N. b. m., 16 hands, star and snip, foaled 1853 ; got by Neave's Cassius M. Clay, Jr.

First dam, by Diamond, son of American Eclipse.

Second dam, a running mare, said to be thoroughbred.

Cassius M. Clay, Jr. (Neave's), by Cassius M.

Clay, son of Henry Clay, by Andrew Jackson.

First dam, by Chancellor, son of Mambrino.

Second dam, by Engineer 2d, son of Engineer, by imp. Messenger. 


\section{No. 26.}

LADY NODINE. hr. m., 15.1 hands, white hinds heels, foaled 1863; got by Rysdyk's Hambletonian.

Dam, Montgomery Maid, by Seely's American Star.

Hambletonian (Rysdyk's), by Abdallah.

First dam, Charles Kent Mare, by imp.

Bellfounder.

Second dam, One Eye, by Bishop's

Hambletonian.

Third dam, Silvertail, by imp. Messenger.

Abdallah, by Mambrino, son of imp. Messenger.

Dam, Amazonia, by son of imp. Messenger.

Hambletonian (Bishop's), by imp. Messenger.

First dam, Pheasant, by imp. Shark.

Second dam, by imp. Medley.

American Star (Seely's), by Stockholm's

American Star, son of Duroc.

First dam, Sally Slouch, by Sir Henry.

Second dam, by imp. Messenger.

No. $2 \%$

LADY CARDINAL. blk. m., 15.1 hands, star and snip, white legs; foaled 1863, got by Harry Clay, son of Neave's Cassius M. Clay, Jr.

First dam, by Cardinal.

Second dam, Harry Clay's dam, by imp.

Bellfounder.

Cassius M. Clay, Jr. (Neave's), by Cassius M. Clay, son of Henry Clay, by Andrew Jackson.

First dam, by Chancellor, son of Mambrino.

Second dam, by Engineer 2d (sire of Lady Suffolk), by imp. Messenger. 
No. 28.

MATTIE. b. m., 15.2 hands, black points, foaled 1866; got

First dam, Lady Earle, by Chauncey Green.

Scc̀ond dam, by Judson's Hambletonian.

Third dam, by Harris' Hambletonian.

Fourth dam, by Judson's Hambletonian.

Fifth dam, by Harris' Hambletonian.

Hambletonian (Rysdyk's), by Abdallah.

First dam, Charles Kent Mare, by imp.

Bellfounder.

Second dam, One Eye, by Bishop's

Hambletonian.

Third dam, Silvertail, by imp. Messenger.

Abdallah, by Mambrino, son of imp. Messenger.

Dam, Amazonia, by son of imp. Messenger.

Hambletonian (Bishop's), by imp. Messenger.

First dam, Pheasant, by imp. Shark.

Secoud dam, by imp. Medley.

Chauncey Green, by Hale's Green Mountain

Morgan.

Dam, by Judson's Hambletonian.

Green Mountain Morgan (Hale's), by Gifford

Morgan, son of Woodbury Morgan.

Dam, by Woodbury Morgan.

Hambletonian (Judson's), by Bishop's

Hambletonian, son of imp. Messenger.

Dam, by Wells' Magnum Bonum.

Hambletonian (Harris'), by Bishop's

Hambletonian, son of imp. Messenger.

Darn, the Messenger Mare, owned by Israel

Munson, of Boston. 
BONNIE LASSIE. b. m., $15.1 \frac{1}{2}$ hands, star and snip, white near hind heel, foaled 1865; got by Rysdyk's Hambletonian.

Hambletonian (Rysdyk's), by Abdallah. First dam, Charles Kent Mare, by imp. Bellfounder.

Second dam, One Eye, by Bishop's

Hambletonian.

Third dam, Silvertail, by imp. Messenger. Abdallah, by Mambrino, son of imp. Messenger.

Dam, Amazonia, by son of imp. Messenger.

Hambletonian (Bishop's), by imp. Messenger.

First dam, Pheasant, by imp. Shark.

Second dam, by imp. Medley.

\section{No. 30.}

LADY DEXTER. b. m., 15 hands, strip in face, white legs, (Full Sister to foaled 1861; got by Rysdyk's Hambletonian.

Dam, Dexter's dam, by Secly's American Star.

Hambletonian (Rysdyk's), by Abdallah.

First dam, Charles Kent Mare, by imp. Bellfơnder.

Second dam, One Eye, by Bishop's Hambletonian

Third dam, Silvertail, by imp. Messenger. Abdallab, by Mambrino, son of imp. Messenger.

Dam, Amazonia, by son of imp. Messenger. Hambletonian (Bishop's), by imp. Messenger.

First dam, Pheasant, by imp. Shark.

Second dam, by imp. Medley. American Star (Seely's), by Stockholm's American Star, son of Duroc.

First dam, Sally Slouch, by Sir Henry. Second dam, by imp. Messenger. 


\section{No. 31.}

EMMA MILLS. ch. m., 15.1 hands, strip in face, white (Dam of
ndependent.") American Star.

Dam, by Radiker's Alexander W., son of Alexander, thoroughbred from Virginia.

\section{No. 32.}

LEDGER GIRL. br. m., 15.1 hands, small star, black points, foaled 1863; got by Rysdyk's Hambletonian.

Darn, by son of Barney's Henry, son of Edward Long's Henry 2 d.

Hambletonian (Rysdyk's), by Abdallah.

First dam, Charles Kent Mare, by imp.

Bellfounder.

Second dam, One Eye, by Bishop's

Hambletonian

Third dam, Silvertail, by imp. Messenger. Abdallah, by Mambrino, son of imp. Messenger.

Dam, Amazonia, by son of imp. Messenger. Hambletonian (Bishop's), by imp Messenger.

First dam, Pheasant, by imp. Shark.

Second dam, by imp. Medley.

Henry 2d (Long's), by Sir Henry, son of

Sir Archy.

First dam, the dam of Sir Lovell, by imp.

Light Infantry.

Second dam, by imp. Messenger.

Third dam, by Bashaw, son of imp. Wildair.

Fourth dam, by True Britton, son of imp

Othello.

Fifth dam, by imp. Sterling. 
No. 33.

GLENCOE MAID. ch, m., 15.1 hands, strip in face, near white hind ankle, foaled 1855 .

\section{No. 34.}

STELLA. b. m., $14 \frac{1}{2}$ hands, strip in face, white near fore heel, white near hind ankle.

\section{No. 35.}

LADY FALIIS. b. m., 15.3 hands, strip in face, foaled (Dam of Socrates.) 1859 ; got by Seely's American Star.

Dam by L. I. Black Hawk.

American Star (Seely's), by Stockholm's

American Star, son of Duroc.

First dam, Sally Slouch, by Sir Henry.

Second dam, by imp. Messenger.

Black Hawk (Long Island), by Andrew .

Jackson, son of Young Bashaw, by Grand

Jiashaw.

Dam, Sally Miller, by Mambrino, son of imp.

Messenger.

\section{No. 36.}

WELLS' STAR. blk. m., 15.1 hands, star and snip, white off hind heel, foaled 1855; got by Seely's American Star.

Dam by Bertrand, son of Kentucky Bertrand.

American Star (Secly's), by Stockholm's

American Star.

First dam, Sally Slouch, by Sir Henry.

Second dam, by imp. Messenger. 
No. $3 \%$.

NELLIE SAYRE. br. m., 15.1 hands, star, white off hind heel, foaled 1853; got by Seely's American Star.

First dam, by Hulse's Hickory.

Second dam, by Lattourett's Bellfounder. American Star (Seely's), by Stockholm's American Star, sun of Duroc. First dam, Sally Slouch, by Sir Henry. Second dam, by imp. Messenger.

EMMA HOYT. ch. m., $15 \frac{1}{2}$ hands, star and snip, white near hind heel, foaled 1859; got by Seely's American Star.

First dam, by Ohio Eclipse, son of American Eclipse.

Second dam, a mare of Messenger descent. American Star (Seely's), by Stockholm's American Star, son of Duroc. First dam, Sally Slouch, by Sir Henry. Second dam, by imp. Messenger. 
No. 39.

HATTIE HOGAN. br. m., 15.3 hands, star and snip, white hind heels, foaled 1867; got by Harry Clay.

First dam, Nellie Sayre, by Seely's American

Star.

Second dam, by Hulse's Hickory.

Third dam, by lattourett's Bellfounder.

Harry Clay, by Neave's Cassius M. Clay, Jr.

Dam, by imp. Bellfounder.

Cassius M. Clay, Jr. (Neave's), by Cassius M.

Clay.

First dam, by Chancellor, son of Mambrino.

Second dam, by Engineer 2d, sire of Lady Suffolk.

American Star (Seely's), by Stockholm's

American Star, son of Duroc.

First dam, Sally Slouch, by Sir Henry.

Second dam, by imp. Messenger.

No. 40 .

ADELE. b. m., $15 \frac{1}{2}$ hands, star and snip, white hind ankles, white near fore heel, foaled 1867; got by Rysdyk's Hambletonian.

Dam, Lady Franklin (owned by David Bonner, Esq.)

Hambletonian (Rysdyk's), by Abdallah.

First dam, Charles Kent Mare, by imp.

Bellfounder.

Second dam, One Eye, by Bishop's

Hambletonian.

Third dam, by imp. Messenger.

Abdallah, by Mambrino, son of imp. Messenger.

Dam, Amazonia, by son of imp. Messenger. Hambletonian (Bishop's), by imp. Messenger.

First dam, Pheasant, by imp. Shark.

Second dam, by imp. Medley. 
No. 41.

JANE MURRAY. gr, m., 15.1 hands, foaled 1853.

(Dam of Lottery.)

No. 42.

KITTIE HARKER. b. m., 15.2 hands, strip in face, white off hind heel, white spot on off fore heel, foaled 1863; got by Ethan Allen, son of Hill's Vermont Black Hawk.

First dam, Martha, by Abdallah.

Second dam, by Young Bellfounder.

Third dam, by Corn Cracker.

Fourth dam, by Hickory.

Abuallah, by Mambrino, son of imp. Messenger.

Dam, Amazonia, by son of imp. Messenger.

Bellfounder (Young), by imp. Bellfounder.

Dam, by Wildair, son of Decatur. 
No. 43.

FAIRY. b. m., 15.2 hands, white hind ankles, foaled 1865 ; got by Rysdyk's Hambletonian.

First dam, Emma Mills, by Seely's American Star.

Second dam, by Radiker's Alexander W.,

- son of Alexander (thoroughbred from Virginia).

Hambletonian (Rysdyk's), by Abdallah.

First dam, Charles Kent Mare, by imp.

Bellfounder.

Second dam, One Eye, by Bishop's

Hambletonian.

Third dam, Silvertail, by imp Messenger.

Abdallah, by Mambrino, son of imp. Messenger.

Dam, Amazonia, by son of imp. Messenger.

Hambletonian (Bishop's), by imp. Messenger.

First dam, Pheasant, by imp. Shark.

Second dam, by imp. Medley.

American Star (Seely's), by Stockholm's

American Star, son of Duroc.

First dam, Sally Slouch, by Sir Henry.

Second dam, by imp. Messenger. 


\section{No. 44.}

BJOSSOM. b. m., 15.2 hands, small star, white near hind (Full Sister of
Idol and Gazelle.) heel, foaled May 1st, 1868; got by Rysdyk's Hamble tonian.

First dam, Hattie Wood, by Harry Clay.

Second dam, Grandmother, by Terror. Hambletonian (Rysdyk's), by Abdallah.

First dam, Charles Kent Mare, by imp. Bellfounder.

Second dam, One Eye, by Bishop's Hambletonian.

Third dam, Silvertail, by imp. Messenger. Abdallab, by Mambrino, son of imp. Messenger.

Dam, Amazonia, by son of imp. Messenger. Hambletonian (Bishop), by imp. Messenger.

First dam, Pheasant, by imp. Shark.

Second dam, by imp. Medley.

Harry Clay, by Neave's Cassius M. Clay, Jr.

Dam, by imp. Bellfounder.

Cassius M. Clay, Jr. (Neave's), by Cassius M.

Clay, son of Henry Clay, by Andrew Jackson, son of Young Bashaw, by Grand Bashaw.

First dam, by Chancellor, son of Mambrino.

Second dam, by Engineer 2d, son of

Engineer, by imp. Messenger.

Terror, by American Eclipse.

First dam, Lady Lightfoot, by Sir Archy. Secoud dam, Black Maria, by imp. Shark. Third dam, by imp. Clockfast.

Fourth dam, Burwell's Maria, by Regulus. Fifth dam, Camilla, by imp. Fearnaught. Sixth dam, Calesta, by Forrester. 
No. 45.

LADY DA VIES. eh. m., 15.2 hands, strip in face, white hind legs, foaled got by Long Island Black Hawk.

Dam, by Abdallah.

Black Hawk (Long Island), by Andrew Jackson, son of Young Bashaw, by Grand Bashaw.

Dam, Sally Miller, by Mambrino, son of imp. - Messenger.

Abdallah, by Mambrino, son of imp. Messenger. Dam, Amazonia, by son of imp. Messenger.

No. 46.

LADY GALATION. ch. m., 15.3 hands, star, white near hind ankle, foaled 1854; got by Seely's American Star.

American Star (Seely's) by Stockholm's American Star, son of Duroc.

First dam, Sally Slouch, by Sir Henry.

Second dam, by imp. Messenger.

No. 47.

VIOLET. b. m., 15.2 hands, black points, foaled March 16th, 1868; got by Rysdyk's Hambletonian.

First dam, Sallie Feagles, by Smith's Clay, son of Neave's Cassius M. Clay, Jr.

Second dam, by Hickory.

Third dam, of Messenger descent.

Hickory, by Hickory, son of imp. Whip.

First dam, by Mambrino, son of imp.

Messenger.

Second dam, by imp. Highlander. 
No. 48.

LADY TALMADGE. ch. m., 15.1 hands, strip in face, white off hind ankle, foaled 1853; got by Seely's American Star.

Dam, from Western New York. American Star (Seely's), by Stockholm's American Star, son of Duroc.

First dam, Sally Slouch, by Sir Henry.

Second dam, by imp. Messenger.

No. 49.

UARY SANDFORD. b. m., 15 hands, white near heels, foaled 186\%; got by Plunket Horse.

Dam, Lady Sanford, dam of Jay Gould, by Seely's American Star.

Plunket Horse, by Alexander's Abdallah.

Dam, by Bay Richmond.

Abdallah (Alexander's), by Rysdyk's

Hambletonian.

First dam, Katy Darling, by Bay Roman.

Second dam, by Mambrino, son of Mambrino. Hambletonian (Rysdyk's), by Abdallah.

First dam, Charles Kent Mare, by imp.

Bellfounder.

Second dam, One Eye, by Bishop's

Hambletonian.

Third dam, Silvertail, by imp. Messenger. Abdallah, by Mambrino, son of imp. Hi essenger.

Dam, Amazonia, by son of imp. Messenger. Hambletonian (Bishop's), by imp. Messenger.

First dam, Pheasant, by imp. Shark.

Second dam, by insp. Medley.

Bay Richmond, by Toby, son of imp. Brown

Highlander

Dam, Lady Pluck, by Mambrino.

American Star (Seely's), by Stockholm's

American Star, son of Duroc.

First dam, Sally Slouch, by Sir Henry.

Second dam, by imp. Messenger. 
No. 50.

FANNY HARRIS. ch. m., 15.2 hands, small star in forehead, foaled 1853; got by Seely's American Star.

First dam, by Black Rock, son of Roebuck.

Second dam, by Walden Messenger.

Third dam, foaled 1798, by imp. Messenger.

American Star (Seely's), by Stockholm's

American Star, son of Duroc.

First dam, Sally Slouch, by Sir Henry.

Second dam, by imp. Messenger.

No. 51.

HOE MARE. b. m., 15.3 hands, strip in face, black points, foaled 1854; got by Wilson's Sir Henry.

First dam, by American Eclipse.

Second dam, by Redbird, son of Bishop's Hambletonian. 
No. 52.

ANTOINETTE. b. m., 15.2 hands, star, white off hind leg, foaled 1864 ; got by Shepherd's Rattler

First dam, by Young Isulle Rock, son of Edward Long's Eclipse.

Second dam, by Edward Long's Henry 2d. Rattler (Shepherd's), by Biggart's Rattler.

Dam, by Edward Long's Tornado.

Rattler (Biggart's), by Sir Henry, son of the Garret Horse.

Dam, by Hyde Horse, son of Edward Long's Magnum Bonum.

Tornado (Long's), by American Eclipse, son of Duroc.

First dam, P.olly Hopkins by Virginian.

Second dam, Janey, by imp. Archduke.

Third dam, bv imp. Stirling.

Fourth dam, by imp. Obscurity.

Fifth dam, Miss Slamerkin, by imp. Wildair.

Eclipse (Long's), by American Eclipse, son of Duroc.

First dam, by Bajazet, son of imp. Tanner. Second dam, by Mereury, son of imp. Janus.

Third dam, by imp. Messenger.

Fourth dam, by Badger, son of imp. Badger. Henry 2d (Long's), by Sir Henry, son of Sir Archy.

First dam, the dam of Sir Lovel, by imp.

Light Infantry.

Second dam, by imp. Messenger.

Third dam, by Bashaw, son of imp. Wildair.

Fourth dam, by True Britton, son of imp.

Othello.

Fifth dam, by imp Sterling. 
No. 53.

GLENELLA. ch. m., 15.2 hands, strip in face, white hind legs, foaled 1864; got by Woodward Star, son of Seely's American Star.

Dam (the dam of Green Mountain Maid).

No. 54.

ROSETTA. b. m., 15.1 hands, little white on hind heels, foaled April 22d, 1867, got by Rysdyk's Hambletonian.

Dam, Grey Rose, by Harris' Hambletonian. Hambletonian (Rysdyl's), by Abdallah.

First dam, Charles Kent Mare, by imp.

Bellfounder.

Second dam, One Eye, by Bishop's

Hambletonian.

Third dam, Silvertail, by imp. Messenger. Abdallah, by Mambrino, son of imp. Messenger.

Dam, Amazonia, by son of imp. Messenger. Hambletonian (Bishop's), by imp. Messenger.

First dam, Pheasant, by imp. Shark.

Secand dam, by imp. Medley.

Hambletonian (Harris') by Bishop's

Hambletonian, son of imp. Messenger.

Dam, the mare owned by Israel Munsen, of

Boston, and represented to be by imp.

Messenger. 
MINEOLA. b. m, star in forehead, spot on nose, white near hind ankle, foaled May 1868 ; got by Rysdyk's Hambletonian.

First dam, Lady Banker, by Roe's Abdallah Chief.

Second dam, by Saltram.

Hambletonian (Rysdyk's), by Abdallah.

First dam, ('harles Kent Mare, by imp. Bellfounder.

Second dam, One Eye, by Bishop's Hambletonian.

Third dam, Silvertail by imp. Messenger. Abdallah, by Mambrino, son of imp. Messenger. Dam, Amazonia, by son of imp. Messenger.

Hambletonian (Bishop's), by imp. Messenger.

First dam, Pheasant, by imp. Shark.

Second dam, by imp. Medley

Abdallah Chief (Roe's), by Abdallah, son of Mambrino.

First dam, by Philip's, son of Duroc.

Second dam, by Decatur, thoroughbred son of Henry.

Saltram, ly Webber's Kentucky Whip, son of Blackburn's Whip.

First dam, by Sea Gull, son of Duroc.

Second dam, by Camptown, son of Icopold.

Whip (Blackburn's), by imp. Whip.

First dam, Speckleback, by Celcr.

Second dam, Speckleback, by Celer.

Third dam, by Pilgrim.

Fourth dam, by imp. Janus.

Fifth dam, by imp. Valiant.

Sixth dam, by imp. Jolly Roger.

Seventh dam, by imp. Shock.

Eighth dam, by imp. Sober John.

Leupold, by Ogle's Osear, son of imp. Gabriel.

First dam, Katydid, by imp. Expidition.

Second dam, by imp. Sourcrout.

Third dam, Matchless. by imp. Slender.

Fourth darn, Fair American, by Traveller.

Fifth dam, Miss Slamerkin, by imp. Wildair. 


\section{SEVEN YEARS OLD MARES.}

No. 1.

LADY MOSCOW. rn. m., black points, foaled August 5th, 1866 ; got by Rysdyk's Hambletonian.

First dam, Roan Marie.

\section{No. 2.}

AMAND A. b. m., star and snip, little white on off hind heel, foaled 1866 ; got by Rysdyk's Hambletonian.

First dam, by Cardinal.

Second dam, Rhoda, by One Eyed Kentucky Hunter.

No. 3.

FLORA FEAGLES. b. m., star, black points, foaled 1866 ; got by Rysdyk's Hambletonian.

First dam, Sallie Feagles, by Smith's Clay, son of Neave's Cassius M Clay, Jr.

Second dam, by Hickory.

Third dam, of Messenger descent. 
No. 4.

LADY HII.L. b. m., star and snip, little white on hind heels, foaled October 22, 1866; got by livsdyk's Hambletonian.

First dam, by Harry Clay, son of Neave's Cassius M. Clay, Jr.

Second dam, by Bertrand.

No. 5.

ROSEOLA. b. m., white off hind heel, foaled, 1866; got by Rysdyk's Hambletonian.

First dam, Lady Van Buren, by Redbird.

Second dam, by Young Bellfounder, son of imp. Bellfounder.. 


\section{SIX YEARS OLD MARES.}

No. 1.

RACOLA. br. m., white hind heels, foaled April 22d, 1867 ; got by Rysdyk's Hambletonian.

Dam, Brown Pacer.

No. 2.

CALYPSO. b. m., small spot on nose, white hind ankles, white fore heels, white hairs in tail, white spot on near side, foaled Tuly 4, 1867; got by Rysdyk's Hambletonian.

Dam, Kate Smith, by Abdallah, son of Mambrino.

No. 3.

JOSEPHINE. b. m., star, white hind ankles, foaled August 13th, 1867; got by Rysdyk's Hambletonian.

Dam, Lucy Almack, by Young Engineer, son of Engineer 2d, sire of Lady Suffolk.

No. 4.

GAY SPANKER. rn. m., black points, foaled July 9th, 1867 ; got by Rysdyk's Hambletonian.

First dam, Roan Marie. 


\section{No. 5.}

BESS. b. $m$, star and snip, white near ankles, brown spot behind the shoulder, foaled August 16, 1867 ; got by Rysdyk's Hambletonian.

First dam, Jessie Sayre, by Harry Clay, son of Neave's Cassius M. Clay, Jr.

Second dam, by Liberty, son of Lance.

\section{No. 6.}

ZENOBIA. br. m., black points, foaled September 18, 1867; got by Rysdyk's Hambletonian.

First dam, Belle, by Post Hambletonian, son of Rysdyk's Hambletonian.

Second dam, by Ethiopian Prince, son of Wildair.

Third dam, from Ohio.

\section{No. $\%$.}

DUCHESS MAY. b. m., strip in face, white near fore heel, white hind legs, foaled 1867; got by Rysdyk's Hambletonian.

First dam, by Jupiter, son of L. I. Black Hawk.

Second dam, Annie Laurie, by Harris' Hambletonian, son of Bishop Hambletonian, by imp. Messenger. 


\section{FIVE YEARS OLD MARES.}

\section{No. 1.}

ZERLINA. b. m., little white on near hind heel, foaled April 19, 1868; got by Rysdyk's Hambletonian. First dam Annie Seely, by Genl. Gifford, son of Gifford Morgan.

Second dam, by Abdallah, son of Mambrino. Third dam, by Webber's Tom Thumb. Fourth dam, Charles Kent Mare (dam of Rysdyk's Hambletonian), by imp.

Bellfounder.

No. 2.

QUEEN OF MAY. b. m., white near hind ankle, white off hind heel, foaled May 1, 1868; got by Rysdyk's Hambletonian.

First dam, Lady Overton, by Abdallah, son of Mambrino.

Second dam, by Mount Holly, son of imp.

Messenger.

No. 3.

STARBEAM. b. m, star, white on lip, white near fore ankle, white hind ankles, foaled June 12, 1868 ; got by Rydsyk's Hambletonian.

Dam, Montgomery Maid, by Seely'y American Star. 
No. 4.

UNDINE. b. m., white near hind ankle, foaled June 26, 1868; got by Rysdyk's Hambletonian.

First dam, Roan Marie.

No. 5 .

LAVINIA. kr. m., black points, foaled 1868; got by Truesdell's Hambletonian.

First dam, Babbitt Abdallah, (sister to Roe's Abdallah Chief), by Abdallah, son of Mambrino.

Second dam, by Philips, son of Duroc.

Third dam, by Dictator (thoroughbred son of Henry.)

Hambletonian ('Truesdell's), by Welling Colt, (owned by John Linn Newton, N. J.), son of Rysdyk's Hambletonian.

First dam, Juda, by Liberty, son of Lance.

Second dam, by imp. Bellfounder.

No. 7.

MABLE. b. m., star, white fore heels, white near hind ankle, white off hind heel, foaled October, 1868; got by Rysdyk's Hambletonian.

Dam, Lady Franklin, owned by David Bonner, Esq. 


\section{FOUR YEARS OLD MARES.}

No. 1.

IDA WEBSTER. b. m., star, white hind heels, foaled May 24, 1869; got by Rysdyk, son of Rysdyk's Hambletonian.

First dam, Sharpless Abdallah, by Abdallah, son of Mambrino.

Second dam, by One Eyed Kentucky Hunter.

No. 2.

SUNBEAM. b. m., white near hind heel, foaled June 20, 1869 ; got by Idol, son of Rysdyk's Hambletonian.

Dam, Lady Packer, by Witherell Messenger, son of Winthrop Messenger, by imported Messenger. 


\title{
THREE YEARS OLD MARES.
}

\author{
No. 1.
}

FINESSE. $b$ m., white off hind ankle, foaled April 23, 1870 ; got by Pierson, son of Rysdyk's Hambletonian.

First dam, Bonnie Lassie, by Rysdyk's Hambletonian.

Second dam, by Fox Hunter.

No. 2.

CHARM. b. m., star and snip, white hind ankles, foaled May

1, 1870, got by Idol, son of Rysdyk's

Hambletonian.

First dam, Satinet (dam of Messenger Duroc), by Roe's Abdallah Chief.

Second dam, Catbird, by Whistle Jacket. Third dam, Lyon Mare, by Bertholf Horse. Fourth dam, by Duroc, son of imp. Diomed. Abdallah Chief (Roe's), by Abdallah, son of Mambrino.

First dam, by Philips, son of Duroc.

Second dam, by Decatur, thoroughbred, son of Henry.

Whistle Jacket, by Mambrino, son of imp.

Messenger.

First dam, by American Eclipse.

Second dam, by Bertholf Horse.

Bertholf Horse, by imp. Messenger.

Dam, by Duroc. 
No. 3.

LUCETT A. b. m., white hind ankles, white near fore beel, foaled May 6th, 1870; got by Rysdyk's Hambletonian.

Dam, Lucy Almack, by Young Engineer, son. of Engineer 2d, sire of Lady Suffolk.

No. 4.

LADY BELMONT. br. m., strip in face, white near ankles, foaled May 7, 1870; got by Rysdyk's

Hambletonian.

Dam, Kate, dam of Bruno.

No. 5.

EMMA FRANCES. br. m., white off hind heel, foaled May : $(6,1870$, got by Messenger Duroc, son of Rysdyk's Hambletonian.

Tirst dam, Mildred, by Rysdyk's

Hambletonian.

Second dam, Linda (the old trotting m:rre), by Abel Horse, son of Smith's Napoleon, by Napoleon, son of Young Mambrino, by Chancellor, son of Mambrino, by imp. Messenger. 


\section{No. 6.}

ZULU. b. m., star, spot on nose, black points, foaled June 9, 1870, got by Pierson, son of Rysdyk's Hambletonian.

First dam, Amanda, by Rysdyk's

Hambletonian.

Second dam, by Cardinal.

Third dam, by One Eyed Kentucky Hunter.

No. 7.

CHEER. b. m., black points, foaled June 10, 1860 ; got by Rysdyk's Hambletonian.

First dam, Jennie Lewis, by Illack Bashaw.

No. 8.

CAROL. br. m., white off fore ankle, white off hind heel ; foaled August 3d, 1870 ; got by Waverly, son of Rysdyk's Hambletonian.

Dam, Lady Farr, by Abdallah, son of Mambrino. 


\section{TWO YEARS OLD FILLIES.}

No. 1.

CARESS. b. f., star in forehead, spot on nose, white hind ankles, foaled April 11, 1871, got by Abdallah Star, son of American Star, Jr.

First dam, Lady Bryant, by Brignoli, son of Mambrino Chief.

Second dam, by Cracker, son of Boston (the sire of Lexington).

No. 2.

XANTIPPE. b. f, star and snip, white hind heels, foaled April 15, 1871, got by Rysdyk's Hambletonian. First dam, Lady Fallis, (dam of Socrates), by Seely's American Star.

Second dam, by Long Island Black Hawk, son of Andrew Jackson, by Young liashaw.

\section{No. 3.}

ElINOR. ch. f., strip in face, white near hind ankle, white off hind heel, foaled May 11, 1871; got by A bdallah Star, son of American Star, Jr.

First dam, Mistress Henyon, by Roe's

Abdallah Chief, son of Abdallah, by

Mambrino.

Ser:ond dam, by Sir Henry. 


\section{No. 4.}

FLORETTA. ch. $\mathrm{f}$, strip in face, white hind legs, foaled May 16, 1871, got by Messenger Duroc, son of Rysdyk's Hambletonian.

First dam, Sallie Feagles, by Smith's Clay, son of Neave's Cassius M, Clay, Jr.

Second dam, by Hickory.

Third dam of Messenger descent.

\section{No. 5.}

GLENROSE. b. f., star and snip, white hind ankles, foaled May 25, 1871; got by Idol, son of Rysdyk's Hambletonian.

First dam, Lady Durland, by Seely's American Star.

Second dam, by Nigger Lance, son of Lance, by American Eclipse.

No. 6.

LORRAINE. b. f, white hind heels, foaled May 26, 1871; got by Hilessenger Duroc, son of Rysdyk's Hambletonian.

First dam, Lady Nodine, by Rysdyk's Hambletonian.

Second dam, Muntgomery Maid, by Seely's American Star. 
No. 7.

BLOOMING. b. f., white face, white fore ankles, white near hind heel, foaled May 28th, 1871, got by Messenger Duroc, son of Rysdyk's Hambletonian.

First dam, Lady Cardinal, by Harry Clay son of Neave's Cassius M. Clay, Jr.

Second dam, by Cardinal.

Third dam, Harry Clay's dam, by imp. Bellfounder.

No. 8.

IDALIA. ch. f., white face, foaled May 29, 1871, got by Abdallah Star, son of A merican Star, Jr.

First dam, Ledger Girl, by Rysdyk's

Hambletonian.

Second dam, by son of Barney's Henry, son of Edward Long's Henry 2d, by Sir Henry.

No. 9.

THERESA. b. f., white off hind heel, foaled .July 15, 1871, got by Messenger Duroc, son of Rysdyk's Hambletonian.

First dam, Kitty Mills, by Seely's American Star.

Second dam, by Wildair, son of Decatur.

No. 10.

ULEM A. b. f., white near hind ankle, white off hind heel, foaled July 24, 1871, got by Post's Conductor, son of Rysdyk's Hambletonian.

Dam, Lady Talmadge, by Seely's American Star. 


\section{YEARLING FILLIES.}

No. 1.

QUEEN BESS. ch. f., strip in face, white off hind heel, foaled April 3d, 1872; got by Idol, son of Rysdyk's Hambletonian.

First dam, Lady Davies, by L. I. Black Hawk. son of Andrew Jackson.

Second dam, by Abdallah, son of Mambrino.

No. 2.

ROSALINDA. br. f., black points, foaled April 5th 1872 ; got by Rysdyk's Hambletonian.

First dam, Nellie Sayre, by Seely's American Star.

Second dam, by Hulse's Hickory.

Third dam, by Lattourett's Bellfounder.

No. 3.

LUCIA. b. f., star, black points, foaled April 19th, 1872 ; got by Speedwell, son of Rysdylz's

Hambletonian.

First dam, Fanny Harris, by Seely's American Star.

Second dam, by Black Rock,

Third dam, by Walden Messeriger.

Fourth dam, by imp. Messenger. 
No. 4.

QUART0. ch. f., white off hind heel, fouled May 10th, 1872; got by Idol, son of Rrsdyk's Hambletonian.

First dam, Emma Hoyt, by Seely's American Star.

Second dam, by Ohio Eclipse.

Third dam, of Messenger descent.

\section{No. 5.}

ADRIANA. b. f., star, white hind ankles, foaled May 14th, 1872 ; got by Messenger Duroc, son of Rysdyk's Hambletonian.

First dam, Kitty Hurker, by Ethan Allen, son of Hill's Vermont Black Hawk.

Secoud dam, Martha, by Abdallah, son of Mambrino.

Third dam, by Young Bellfounder, son of imp. Bellfounder.

Fourth dam, by Corn Cracker.

Fifth dam, by Hickory.

No. 6.

PRIMR0SE. gr. f., star in forehead, foaled May 15th, 1872; got by Messenger Duroc, son of Rysdyk's Hambletonian.

Dam, Grey Rose, by Harris' Hambletonian, son of Bishop's Hambletonian, by imp. Messènger. 
No. $\%$.

EMILIA. b f , small white spot on nose, white hind ankles, foaled May 17th, 1872; yot by Rysdyk's

Hambletonian.

First dam, Lady Bellfounder, by Webber's Tom Thumb.

- Second dam, by Crabtree Bellfounder. Third dam, by imp. Bellfounder.

No. 8.

GLENMARY. b. f., star, whitè hind ankles, foaled May 20th, 1872 ; got by Rysdyk's Hambletonian.

First dam, Fanniestriter by Your Almak, son $\rightarrow$ Alm thatrorino.

Second dam, by Jonas Flowers Horse

sportsman, by Chatrectors son of

Nembrino.

No. 9.

IONA. ch. f., strip in face, white hind legs, white off fore heel, foaled May 22d, 1872; got by Abdallah Star, son of Americau Star, Jr.

First dam, Lady Nodine, by Rysdyk's Hambletonian.

Second dam, Montgomery Maid, by Secly's American Star. 
No. 10.

MIRANDA. ch. f., strip in face, white hind legs, foaled May 25th, 1872; got by Messenger Duroc, son of Rysdyk's Hambletonian.

First dam, Green Mountain Maid, (dam of Prospero), by Harry Clay, son of Neave's Cassius M. Clay, Jr.

\section{No. 11.}

CLARABEL. b. f., white off hind ankle, foaled May 30th, 1872; got by Abdallah Star, son of American Star, Jr.

First dam, Fairy, by Rysdyk's Hambletonian.

Second dam, Emma Mills, by Seely's American Star.

Third dam, by Radiker's Alexander W., son of Alexander, (thoroughbred from Virginia.)

\section{No. 12.}

DORETTA. ch. f., strip in face, white fore heels, white near hind leg, white off hind heel, foaled June 17th, 1872; got by Messenger Duroc, son of Rysdyk's Hambletonian.

Dam, Lady Packer, by Witherell Messen fुer, son of Wintlirop Messenger, by imp Messenger. 


\section{No. 13.}

MARY GRAFTON. br. f., star and snip, white off fore ankle, white hind legs, foaled July 19th, 1872 ; got by Rysdyk's Hambletonian.

First dam, Jady Brown, by Seely's American Star.

Second dam, by Nigger Lance, son of Lance, by American Eclipse.

No. 14.

BELLE ROSE. b. f., black points, foaled August 20th, 1872; got by Idol, son of Rysdyk's Hambletonian.

First dam, Virgo, by Roe's Abdallah Chief, son of Abdallah.

Second dam, Catbird, by Whistle Jacket, son of Mambrino.

Third dam, Lyon Mare, by Bertholf Horse, son of imp. Messenger.

Fourth dam, by Duroc, son of imp. Diomed. 


\section{N O T I C E.}

I am now ready to offer a portion of my Stock at Private Sale during the year.

CHARLES BACKMAN. 



$$
\text { I } 874 \text {. }
$$

$$
\begin{gathered}
\text { ORANGE COUNTY, } \\
\text { STONYFORD }
\end{gathered}
$$

New York.

CHARLES BACKMAN. 



\section{PEDIGREE OF}

\section{RYSDYK'S HAIMBLETONIAN.}

Hambletonian, Rysdyk's, got by Abdallah.

First Dam, Charles Kent Mare, by imp. Bellfounder. Second Dam, One Eye, by Bishop's Hambletonian. Third Dam, Silvertail, by imp. Messenger.

Abdallah, by Mambrino, son of imp. Messenger.

Dam, Amazonia, by son of imp. Messenger.

Hambletonian (Bishop's), by imp. Messenger.

First Dam, Pheasant, by imp. Shark.

Second Dam, by imp. Medley. 



\section{STONY FORD STUD,}

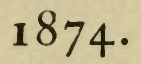

The following Stallions will make the Season of 1874, at the Stony Ford Stud Farm, Orange County, N. Y., the seasun to commence APRIL 1 and close October 1, 1874.

MESSENGER DUROC.-Bay stallion, 16 hands, foaled June 3, 1865; got by Rysdyk's Hambletonian. Firsi dam Satinet (foaled 1854), by Roe's Abdallah Chief; second dam Catbird (foaled 1834), by Whistle Jacket; third dam Lyon Mare, by Bertholf Horse; fourth dam by Duroc, son of imp. Diomed.

Abdallah Chief (Roe's), by Abdallah, son of Mambrino. First dam by Philips, son of Duroc; second dam by Decatur, son of Sir Henry, by Sir Archy.

Whistle Jacket, by Mambrino, son of imp. Messenger. First dam by American Eclipse, son of Duroc; second dam by Bertholf Horse.

Bertholf Horse, by imp. Messenger; dam Beauchamp Mare, by Duroc, son of imp. Diomed.

MESSENGER DURoc's limit of forty mares for season of 1874 , at $\$ 200$, payable in advance, has been filled by the patrons of 1873 . He will be permitted to serve a few additional mares during the season at $\$ 300$, payable in advance.

A BDAI.LA H STAR.-Chestnut stallion, sixteen hands; foaled June 12, 1863; got by American Star, Jr. First dam Curry Abdallah, by Abdallah, son of Mambrino; second dam by imp. Bellfounder; third dam by Royalist; fourth dam by Hardware, son of imp. Messenger.

American Star, Jr., by Seely's American Star, son of Stockholm's American Star, by Duroc; dam by Friday, son of imp. Trustee.

IDOL.-Bay stallion, fifteen hands two and a-half inches; foaled June 9, 1864; got by Rysdyk's Hambletonian; first dam Hattie Wood, by Harry Clay; second dam Grandmother, by Terror, son of American Eclipse.

Harry Clay, by Neave's Cassius M. Clay, Jr., son of Cassius M. Clay, by Henry Clay, son of Andrew Jackson; dam by imp. Bellfounder.

BOLTON.-Bay stallion, sixteen hands; foaled June 13, 1867; got by Rysdyk's. Humbletonian; dam Montgomery Maid, by Seely's American Star.

ELECTIONEER.-Bay stallion, fifteen bands two inches; foaled May 2, 1868; got by Rysdyk's Hambletonian; dam Green Mountain Maid (dam of Prospero and Dame Trot), by Harry Clay, son of Neave's Cassius M. Clay, Jr.

The above stallions are limited to forty mares each, at $\$ 100$ the season, payable in advance.

ELLW OOD, by Messenger Duroc.-Ellwood, Bay stallion, fifteen hands three inches; foaled July 14, 1870; got by Messenger Duroc. First dam Virgo (foaled 1855, full sister to Satinet), by Roe's Abdallah Chief; second dam Catbird (foaled 1834), by Whistle Jacket; third dam Lyon Mare, by Bertholf Horse; fourth dam by Duroc, son of imp. Diomed.

Messenger Duroc, by Rysdyk's Hambletonian. First dam Satinet (foaled 1854), by Roe's Abdallah Chief; secoud dan Catbird (foaled 1834), by Whistle Jacket; third dain Lyon Mare, by Bertholf Horse; fourth dam by Duroc, son of imp. Diomed.

Abdallat Chief (Roe's), by Abdallah, son of Mambrino. First dam by Philips, son of Duroc; serond dam by Decatur, son of Sir Henry, by Sir Archy.

Whistle Jacket, by Mambriuo, son of imp. Messenger. First dam by American Eclipse, son of Duroc; second dam by Bertholf Horse.

Bertholf Horse, by imp. Messenger; dam Beauchamp Mare, by Duroc, son of imp. Diomed.

RIDGEWOOD.- Bay stallion, sixteen bands; foaled May, 1870; got by Rysdyk's Hambletonian. Finst dam Hoe Mare, by Wilson's Sir Henry, son of Sir Henry, by Sir Archy ; second dam by Eclipse, son of Crocker's Eelipse ; third dam by Redbird.

Ellwood And RIDGewood will be limited to twenty-five mares each at $\$ 50$ the season, payable in advance. 



\section{STONY FORD STUD.}

\section{STALLIONS.}

ABDALLAH STAR. ch. s., 16 hands, small star, white off hind ankle, foaled June 12, 1863; got by American Star, Jr.

First Dam, Curry Abdallah, by Abdallah.

Second Dam, by imported Bellfounder.

Third Dam, by Royalist.

Fourth Dam, by Hardware, son of imp. Messenger.

American Star, Jr., by Seely's American Star.

Dam by Friday, son of imp. Trustee.

American Star (Seely's), by Stockholm's American Star, son of Duroc.

First Dam, Sally Slouch, by Sir Henry.

Second Dam, by imp. Messenger.

Friday, by imp. Trustee.

First Dam, Saluda, by Timoleon.

Second Dam, by imp. Sir Harry.

Third Dam, Highland Mary (sister to Sir Archy), by imp. Diomed.

Trustee (imp.), by Catton.

First Dam, Emma, by Whisker.

Second Dam, Gibside Fairy, by Hermes.

Third Dam, Vicissitude, by Pipator.

Fourth Dam, Beatrice, by Sir Peter.

Fifth Dam, Pyrrha, by Matchem.

Sixth Dam, Duchess, by Whitenose.

Seventh Dam, Miss Slamerkin, by Young True Blue.

Eighth Dam, by Lord Oxford's Dun Arabian.

Ninth Dam, D'Arey's black legged Royal Mare.

Abdallah, by Mambrino, son of imp. Messenger.

Dam Amazonia, by son of imp. Messenger. 
IDOL. b. s., $15.2 \frac{1}{2}$ hands, white near fore heel, white hind ankles, foaled June 9, 1864; got by Rysdyk's Hambletonian.

First Dam, Hattie Wood, by Harry Clay. Second Dam, Grandmother, by Terror. Hambletonian (Rysdyk's), by Abdallah.

First Dam, Charles Kent Mare, by imp. Bellfounder.

Second Dam, One Eye, by Bishop's Hambletonian.

Third Dam, Silvertail, by imp. Messenger.

Abdallah, by Mambrino, son of imp. Messenger.

Dam, Amazonia, by son of imp. Messenger.

Hambletonian (Bishop's), by imp. Messenger.

First Dam, Pheasant, by imp. Shark.

Second Dam, by imp. Medley.

Harry Clay, by Neave's Cassius M. Clay, Jr.

Dam, by imp. Bellfounder.

Cassius M. Clay Jr. (Neave's), by Cassius M. Clay, son of Henry Clay, by Andrew Jackson, son of Young Bashaw.

First Dam, by Chancellor, son of Mambrino.

Second Dam, by Engineer 2d (sire of Lady Suffolk). Engineer 2d, by Engineer, son of imp. Messenger.

First Dam, by Plato.

Second Dam, by Rainbow.

Plato, by imp. Messenger.

First Dam, Pheasant, by imp. Shark.

Second Dam, by imp. Medley.

Rainbow, by imp. Wildair.

First Dam, by General Heard's True Briton.

Second Dam, an imp. mare, by Duke of Bolton's Starling.

Terror, by American Echipse, son of Duroc.

First Dam, Lady Lightfoot, by Sir Archy.

Second Dam, Black Maria, by imp. Shark.

Third Dam, by imp. Clockfast.

Fourth Dam, Burwell's Maria, by Regulus.

Fifth Dam, Camilla, by imp. Fearnaught.

Six th Dam, Calista, by Forrester. 
MESSENGER DUROC. b. s., 16 hands, white hind ankles, foaled June 3, 1865; got by Rysdyk's Hambletonian.

First Dam, Satinet, foaled 1854; by Roe's Abdallah Chief.

Second Dam, Catbird, foaled 1834; by Whistle Jacket. Third Dam, Lyon Mare, by Bertholf Horse. Fourth Dam, by Duroc, son of imp. Diomed.

Hambletonian (Rysdyk's), by Abdallah.

First Dam, Charles Kent Mare, by imp. Bellfounder.

Second Dam, One Eye, by Bishop's Hambletonian.

Third Dam, Silvertail, by imp. Messenger.

Abdallah, by Mambrino, son of imp. Messenger.

Dam, Amazonia, by son of imp. Messenger.

Hambletonian (Bishop's), by imp. Messenger.

First Dam, Pheasant, by imp. Shark.

Second Dam, by imp. Medley.

Abdallah Chief (Roe's), by Abdallah, son of Mambrino.

First Dam, by Philips, son of Duroc.

Second Dam, by Decatur, thoroughbred son of Henry. Whistle Jacket, by Mambrino, son of imp. Messenger.

First Dam, by American Eclipse.

Second Dam, by Bertholf Horse.

Bertholf Horse, by imp. Messenger.

Dam, Beauchamp Mare, by Duroc. 
STONY FORD. br. s., 15.2 hands, little white on near hind heel, foaled May 27, 1866; got by Rysdyk's Hambletonian.

First Dam, Annie Seely, by Gen. Gifford, son of Gifford Morgan, by Woodbury Morgan.

Second Dam, Seely Abdallah, by Abdallah.

Third Dam, Belle, by Webber's 'Tom Thumb.

Fourth Dam, Charles Kent Mare (Dam of Rysdyk's

Hambletonian), by imp. Bellfounder.

Fifth Dam, One Eye, by Bishop's Hambletonian.

Six th Dam, Silvertail, by imp. Messenger.

Abdallah, by Mambrino, son of imp. Messenger.

Dam, Amazonia, by son of imp. Messenger.

Hambletonian (Bishop's), by imp. Messenger.

First Dam, Pheasant, by imp. Shark.

Second Dam, by imp. Medley.

WAVERLEY. br. s., 15.3 hands, star, white hind ankles, white . off fore heel, foaled October 9, 1866; got by Rysdyk's Hambletonian.

First Dam, Susie Roberts, by N. Y. Black Hawk.

Second Dam, the well-known mare of Messenger descent, owned and driven by Captain Roberts, of New York.

Black Hawk (New York), by L. I. Black Hawk.

First Dam, sister to Jack Cade, by Leopold (Arabian).

Second Dam, by Bishop's Hambletonian, son of imp.

Messenger.

Black Hawk (Long Island), by Andrew Jackson, son of

Young Bashaw, by Grand Bashaw.

Dam, Sally Miller, by Mambrino, son of imp. Messenger. 


\section{SEVEN YEARS OLD STALLIONS.}

No. 1.

VICTOR von BISMARCK. b. s., white hind heels, foaled Full Brother to Idol and Gazelle.

April 19, 1867; got by Rysdyk's Hambletonian.

First Dam, Hattie Wood, by Harry Clay.

Second Dam, Grandmother, by Terror.

Harry Clay, by Neave's Cassius M. Clay, Jr.

Dam, by imp. Bellfounder.

Cassius M. Clay, Jr. (Neave's), by Cassius M. Clay, son of Henry Clay, by Andrew Jackson, son of Young Bashaw.

First Dam, by Chancellor, son of Mambrino.

Second Dam, by Engineer 2d, son of Engineer, by imp. Messenger.

Terror, by American Eclipse.

First Dam, Lady Lightfoot, by Sir Archy.

Second Dam, Black Maria, by imp. Shark.

Third Dam, by imp. Clockfast.

Fourth Dam, Burwell's Maria, by Regulus.

Fifth Dam, Camilla, by imp. Fearnaught.

Sixth Dam, Calista, by Forrester.

American Eclipse, by Duroc, son of imp. Diomed.

Dain, Miller's Damsel, by imp. Messenger.

Sir Archy, by imp. Diomed.

Dam, Castianira, by Rockingham. 


\section{No. 2.}

BOLTON. b. s., 16 hands, white off hind heel, foaled June 13, 1867 ; got by Rysdyk's Hambletonian.

Dam, Montgomery Maid, by Seely's American Star. Hambletonian (Rysdyk's), by Abdallah.

First Dam, Charles Kent Mare, by imp. Bellfounder.

Second Dam, One Eye, by Bishop's Hambletonian.

Third Dam, Silvertail, by imp. Messenger.

Abdallah, by Mambrino, son of imp. Messenger.

Dam, Amazonia, by son of imp. Messenger.

Hambletonian (Bishop's), by imp. Messenger.

First Dam, Pheasant, by imp. Shark.

Second Dam, by imp. Medley.

American Star (Seely's), by Stockholm's American Star, son of Duroc.

First Darn, Sally Slouch, by Sir Henry.

Second Dam, by imp. Messenger.

Sir Henry, by Sir Archy, son of imp. Diomed.

First Dam, by imp. Diomed.

Second Dam, Bellona, by Bel-Air.

Third Dam, Indian Queen, by Pilgrim.

Fourth Dam, by imp. Janus.

Fifth Dam, by imp. Jolly Roger.

Sixth Dam, by imp. Valiant.

EXCHANGE. gr. s., star and snip, white hind ankles, foaled July 4, 1867 ; got by Rysdyk's Hambletonian.

Dam, Lady Swazey, by Harris' Hambletonian.

Hambletonian (Harris'), by Bishop's Hambletonian.

Dam, the mare owned by Israel Munson, of Boston, and represented to be by imp. Messenger.

Hambletonian (Bishop's), by imp. Messenger.

First Dam, Pheasant, by imp. Shark.

Second Dam, by imp. Medley. 
No. 4.

KENILWORTH. br. s., black points, foaled Sept. 10, 1867 ; Brother to
Waverley. got by Rysdyk's Hambletonian.

First Dam, Susie Roberts, by N. Y. Black Hawk.

Second Dam, the well-known mare of Messenger descent, owned and driven by Captain Roberts, of New York. Black Hawk (New York), by L. I. Black Hawk.

First Dam, sister to Jack Cade, by Leopold (Arabian.) Second Dam, by Bishop's Hambletonian, son of imp. Messenger.

Black Hawk (Long Island), by Andrew Jackson, son of Young Bashaw, by Grand Bashaw.

Dam, Sally Miller, by Mambrino, son of imp. Messenger. 


\section{SIX YEARS OLD STALLIONS.}

No. 1.

TWILIGHT. b. s., white hind heels, foaled April 20, 1868; got by Rysdyk's Hambletonian.

First Dam, Mary Hulse, by Seely's American Star.

Second Dam, of Messenger descent.

American Star (Seely's), by Stockholm's American Star, son of Duroc.

First Dam, Sally Slouch, by Sir Henry.

Second Dam, by imp. Messenger.

No. 2.

ELECTIONEER. b. s., 15.2 hands, white hind heels, foaled May 2, 1868; got by Rysdyk's Hambletonian.

First Dam, Green Mountain Maid, by Harry Clay. Hambletonian (Rysdyk's), by Abdallah.

First Dam, Charles Kent Mare, by imp. Bellfounder.

Second Dam, One Eye, by Bishop's Hambletonian.

'Third Dam, Silvertail, by imp. Messenger.

Abdallah, by Mambrino, son of imp. Messenger.

Dam, Amazonia, by son of imp. Messenger.

Hambletonian (Bishop's), by imp. Messenger.

First Dam, Pheasant, by imp. Shark.

Second Dam, by imp. Medley.

Harry Clay, by Neave's Cassius M. Clay, Jr.

Dam by imp. Bellfounder.

Cassius M. Clay, Jr. (Neave's), by Cassius M. Clay, son of Henry Clay, by Andrew Jackson.

First Dam, by Chancellor, son of Mambrino.

Second Dam, by Engineer 2d (sire of Lady Suffolk). Engineer 2d, by Engineer, son of imp. Messenger.

First Dam, by Plato, son of imp. Messenger.

Second Dam, by Rainbow.

Plato, by imp. Messenger.

First Dam, Pheasant, by imp. Shark.

Second Dam, by imp. Medley.

Rainbow, by imp. Wildair.

First Dam, by General Heard's 'True Briton.

Second Dam, by Duke of Bolton's Starling. 
No. 3.

BRINEY. b. s., white hind heels, white near fore foot, foaled 1868; got by Mambrino Pilot.

First Dam, Lady Dexter, by Rysdyk's Hambletonian.

Second Dam (Dexter's Dam), by Seely's American Star.

Mambrino Pilot, by Mambrino Chief, son of Mambrino Paymaster.

Dam, by Alexander's Pilot, Jr. 


\section{FOUR YEARS OLD STALLIONS.}

No. 1.

STILLSON. ch. s., white hind ankles, foaled May 21, 1870; got by Messenger Duroc, son of Rysdyk's Hambletonian.

Dam, Grey Rose, by Harris' Hambletonian.

Hambletonian (Harris'), by Bishop's Hambletonian.

Dam, the mare owned by Israel Munson, of Boston, and represented to be by imp. Messenger.

Hambletonian (Bishop's), by imp. Messenger.

First Dam, Pheasant, by imp. Shark.

Second Dam, by imp. Medley.

No. 2.

AUGUST BELMONT. b. s., white hind heels, foaled May 22, 1870; got by Rysdyk's Hambletonian.

First Dam, Miss Wansor, by Jackson's Sir Archy, son of Bay State, by Green Mountain Morgan, son of Gifford Morgan.

Second Dam, by Westchester, son of L. I. Black Hawk. Third Dam, by Abdallah.

Fourth Dam, by Engineer 2d (sire of Lady Suffolk).

Black Hawk (Long Island), by Andrew Jackson, son of Young Bashaw, by Grand Bashaw.

Dam, Sally Miller, by Mambrino.

Abdallah, by Mambrino, son of imp. Messenger.

Dam Amazonia, by son of imp. Messenger.

Engineer 2d, by Engineer, son of imp. Messenger.

First Dam, by Plato.

Second Dam, by Rainbow.

Plato, by imp. Messenger.

First Dam, Pheasant, by imp. Shark.

Second Dam, by imp. Medley.

Rainbow, by imp. Wildair.

First Dam, by Gen. Heard's 'T'rue Briton.

Second Dam, an imported mare, by Duke of Bolton's Starling. 
No. 3.

LEGAL. b.s., star, white off hind heel, white near hind ankle, white near fore leg, foaled July 3d, 1870; got by Rysdyk's Hambletonian.

First Dam, Fannie Fiske, by Young Almack.

Second Dam, by Jonas Flower's horse Sportsman. Almack (Young), by Almack, son of Mambrino.

Dam, by Diamond, son of 'American Eclipse. Sportsman (Jonas Flower's), by Chancellor, son of Mambrino.

Dam, by Sir Henry, son of Sir Archy.

No. 4.

ELLWOOD. b. s. 15.3 hands, star, white hind ankles, foaled July 14th, 1870; got by Messenger Duroc.

First Dam, Virgo (foaled 1855, full sister of Satinet), by Roe's Abdallah Chief.

Second Dam, Catbird (foaled 1834), by Whistle Jacket. Third Dam, Lyon Mare, by Bertholf Horse.

Fourth Dam, by Duroc, son of imp. Diomed.

Messenger Duroc, by Rysdyk's Hambletonian.

First Dam, Satinet (foaled 1854), by Roe's Abdallah Chief.

Second Dam, Catbird (foaled 1834), by Whistle Jacket. Third Dam, Lyon Mare, by Bertholf Horse.

Abdallah Chief (Roe's), by Abdallah, son of Mambrino.

First Dam, by Philips, son of Duroc.

Second Dam, by Decatur, son of Sir Henry, by Sir Archy.

Whistle Jacket, by Mambrino, son of imp. Messenger.

First Dam, by American Eclipse, son of Duroc.

Second Dam, by Bertholf Horse.

Bertholf Horse, by imp. Messenger.

Dam, Beauchamp mare, by Duroc, son of imp. Diomed. 
No. 5.

RIDGEWOOD. b. s., 16 hands, star and snip, white near hind ankle, foaled 1870 ; got by Rysdyk's Hambletonian. First Dam, Hoe Mare, by Wilson's Sir Henry, son of Sir Henry, by Sir Archy.

Second Da:n, by Eclipse, son of Crocker's Eclipse. Third Dam, by Redbird. 


\section{THREE YEARS OLD COLTS.}

No. 1.

QUARTETTE. ch. c., white hind ankles, foaled May 15th, 1871 ; got by Idol, son of Rysdyk's Hambletonian.

First Dam, Emma Hoyt, by Seely's American Star. Second Dam, by Ohio Eclipse, son of American Eclipse. Third Dam, of Messenger descent.

American Star (Seely's), by Stockholm's American Star.

First Dam, Sally Slouch, by Sir Henry.

Second Dam, by imp. Messenger.

No. 2.

PROSPECT. blk. c., star, white hind ankles, foaled June 24th, 1871; got by Messenger Duroc, son of Rysdyk's Hambletonian.

Dam, Montgomery Maid, by Seely's American Star. American Star (Seely's) by Stockholm's American Star, son - of Duroc.

First Dam, Sally Slouch, by Sir Henry.

Second Dam, by imported Messenger.

No. 3.

CORSAIR. b. c., star, white spot on off hind heel, foaled July 18th, 1871; got by Rysdyk's Hambletonian. First Dam, Kate Seely, by Walden Messenger. Second Dam, by Mambrino, son of imp. Messenger. 


\section{TWO YEARS OLD COLTS.}

No. 1.

KING WILLIAM. b. c., white hind ankles, foaled May 19, 1872; got by Rysdyk's Hambletonian.

First Dam, Mary Hulse, by Seely's American Star. Second Dam, of Messenger descent.

No. 2.

PICKERING. b. c., snip on nose, white near fore ankle, white (Brother to
Socrates.) hind ankles, foaled May 23, 1872 ; got by Rysdyk's Hambletonian.

First Dam, Lady Fallis, by Seely's American Star. Second Dam, by Long Island Black Hawk. 


\section{YEARLING COLTS.}

No. 1.

DEAN SAGE. br. c., strip in face, white near fore foot, white hind ankles, foaled March 26, 1873; got by Rysdyk's Hambletonian.

First Dam, Nellie Sayre, by Seely's American Star.

Second Dam, by Hulse's Hickory.

Third Dam, by La Tourett's Bellfounder.

No. 2 .

b. c., white near hind ankle, foaled April 7, 1873; got by Idol, son of Rysdyk's Hambletonian.

First Dam, Trustus Webb, by Seely's American Star. Second Dam, by imp. Trustee.

No. 3.

ch. c., star, white hind ankles, foaled April 26, 1873; got by Idol, son of Rysdyk's Hambletonian.

First Dam, Mattie, by Rysdyk's Hambletonian.

Second Dam, Lady Earle, by Chauncey Green, son of Hale's Green Mountain Morgan.

Third Dam, by Judson's Hambletonian, son of Harris' Hambletonian.

Fourth Dam, by Harris' Hambletonian, son of Bishop's Hambletonian.

Fifth Dam, by Judson's Hambletonian, son of Harris' Hambletonian.

Sixth Dam, by Harris' Hambletonian, son of Bishop's Hambletonian. 
No. 4.

HOGARTH. blk. c., strip in face, white under lip, white off fore leg, white hind legs, foaled May 1, 1873; got by Messenger Duroc, son of Rysdyk's Hambletonian. First Dam, Hattie Hogan, by Harry Clay, son of Neave's Cassius M. Clay, Jr.

Second Dam, Nellie Sayre, by, Seely's American Star. Third Dam, by Hulse's Hickory.

Fourth Dam, by La Tourett's Bellfounder.

No. 5.

SPENCER. ch. c., blaze in face, white hind legs, foaled May 7, 1873 ; got by Messenger Duroc, son of Rysdyk's Hambletonian.

First Dam, Lady Nodine, by Rysdyk's Hambletonian. Second Dam, Montgomery Maid, by Seely's American Star.

No. 6.

b. c., star, white fore heels, white hind ankles, foaled May 17, 1873; got by Messenger Duroc, son of Rysdyk's Hambletonian.

First Dam, Kitty Harker, by Ethan Allen, son of Hill's

Vermont Black Hawk.

Second Dam, Martha, by Abdallah, son of Mambrino.

Third Dam, by Young Bellfounder.

Fourth Dam, by Corn Cracker.

Fifth Dam, by Hickory. 
No. 7.

b. c., blaze in face, white near fore leg, white hind legs, foaled May 28, 1873; got by Rysdyk's Hambletonian.

First Dam, Lady Fallis (Dam of Socrates), by Seely's American Star.

Second Dam, by Long Island Black Hawk.

No. 8.

blk. c., white near hind foot, white off hind ankle, foaled June 8, 1873; got by Messenger Duroc, son of Rysdyk's Hambletonian.

First Dam, Alice Van Duzer, by Seely's American Star. Second Dam, by Messenger Duroc, son of Sir Archy Duroc.

Third Dam, by Paymaster, son of imp. Brown Highlander.

No. 9.

blk. c., blaze in face, white off legs, white near hind foot, foaled June 21, 1878; got by Messenger Duroc, son of Rysdyk's Hambletonian.

First Dam, Lady Cardinal, (Dam of Reform). By Harry Clay, son of Neave's Cassius M. Clay, Jr. Second Dam, by Cardinal.

Third Dam, (Dam of Harry Clay), by imp. Bellfounder. 


\section{No. 10.}

ch. c., white near fore leg, white hind legs, foaled July 7, 1873; got by Idol, son of Rysdyk's Hambletonian.

First Dam, Mistress Henyon, by Roe's Abdallah Chief. Second Dam, by Sir Henry, son of Sir Archy.

br. c., star and snip, white hind ankles, foaled June .

9, 1873; got by Rysdyk's Hambletonian.

Dam, Kate, (Dam of Brunette and Bruno).

No. 12.

b. c., white off hind ankle, white near hind heel, foaled August 22, 1873; got by Bolton, son of Rysdyk's Hambletonian.

First Dam, Mildred, by Rysdyk's Hambletonian.

Second Dam, Linda, by Abel Horse, son of Smith's Napoleon, by Napoleon. 


\section{EIGHT YEARS OLD GELDING.}

ISLAND BOY. b. g., white off heels, foaled 1866; got by Hetzell Hambletonian, son of Rysdyk's Hambletonian. First Dam, Lady Durland, by Seely's American Star. Second Dam, by Nigger Lance, son of Lance, by American Eclipse.

\section{SIX YEARS OLD GELDING.}

SILVER HEEL b. g., white near hind heel, foaled May 9, 1868; got by Rysdyk's Hambletonian.

Dam, Rosa Lee, by Harris' Hambletonian.

Hambletonian (Harris'), by Bishop's Hambletonian, son of imp. Messenger.

Dam, the mare owned by Israel Munson, of Boston, and represented to be by imp. Messenger.

Hambletonian (Bishop's), by imp. Messenger.

First Dam, Pheasant, by imp. Shark.

Second Dam, by imp. Medley. 


\section{FIVE YEARS OLD GELDINGS.}

No. 1.

TRIUMPH. b. g., white hind heels, foaled A pril 26, 1869; got by Idol, son of Rysdyk's Hambletonian.

Dam, Rosa Lee, by Harris' Hambletonian.

Hambletonian (Harris'), by Bishop's Hambletonian.

Dam, the mare owned by Israel Munson, of Boston, and represented to be by imp. Messenger.

Hambletonian (Bishop's), by imp. Messenger.

First Dam, Pheasant, by imp. Shark.

Second Dam, by imp. Medley.

No. 2 .

LUCKNOW. b. g., star and snip, white near hind heel, white off fore heel, foaled May 11, 1869; got by Idol, son of Rysdyk's Hambletonian.

Dam, Grandmother (grandam of Idol), by Terror, son of American Eclipse.

No. 3.

PICKWICK. br. g., star and snip, white hind ankles, little white on near fore heel, foaled June 9, 1869; got by Idol, son of Rysdyk's Hambletonian.

First Dam, Lady Brown, by Seely's American Star. Second Dam, by Nigger Lance, son of Lance, by American Eclipse. 
No. 4.

GEORGE GARDINER. ch. g., star and snip, white hind heels, white near fore heel, foaled September 9, 1869 ; got by Messenger Duroc, son of Rysdyk's Hambletonian.

First Dam, Hattie Wood (Dam of Idol), by Harry Clay. Second Dam, Grandmother, by Terror. Harry Clay, by Neave's Cassius M. Clay, Jr.

Dam, by imp. Bellfounder.

Cassius M. Clay, Jr. (Neave's), by Cassius M. Clay, son of Henry Clay, by Andrew Jackson.

First Dam, by Chancellor, son of Mambrino.

Second Dam, by Engineer 2d, son of Engineer, by imp. Messenger.

Terror, by American Eclipse.

First Dam, Lady Lightfoot, by Sir Archy.

Second Dam, Black Maria, by imp. Shark.

Third Dam, by imp. Clockfast.

Fourth Dam, Burwell's Maria, by Regulus.

Fifth Dam, Camilla, by imp. Fearnaught.

Sixth Dam, Calista, by Forrester.

\section{FOUR YEARS OLD GELDING.}

GLENEIDA. ch. g., star, white hind heels, foaled June 25, 1870 ; got by Messenger Duroc, son of Rysdyk's Hambletonian.

First Dam, Lady Nodine, by Rysdyk's Hambletonian. Second Dam, Montgomery Maid, by Seely's American Star. 


\section{THREE YEARS OLD GELDINGS.}

\section{No. 1.}

TASSO. b. g., star and snip, white hind ankles, foaled April 14, 1871;

got by Waverley, son of Rysdyk's Hambletonian. First Dam, Zuleika, by N. Y. Black Hawk, son of L. I. Black Hawk.

Second Dam, by Mambrino Paymaster, son of Mambrino, by imp. Messenger.

No. 2.

MACLURE. blk. g., star, white off fore foot, white hind ankles, foaled April 21, 1871; got by Messenger Duroc, son of Rysdyk's Hambletonian.

First Dam, Nellie Wilmarth, by Raven, son of Hill's Vermont Black Hawk.

Second Dam, Jennie Lind, by Young Andrew Jackson, son of Andrew Jackson.

Third Dam, Polly Sherman, by Bush Messenger, son of imp. Messenger.

No. 3.

EPICURE. b. g., star in forehead, foaled April 28, 1871; got by Waverley, son of Rysdyk's Hambletonian.

First Dam, Lady Pierson, by Neave's Cassius M. Clay, Jr., son of Cassius M. Clay.

Second Dam, by Diamond, son of American Eclipse. Third Dam, a running mare, said to be thoroughbred. 
No. 4.

ORPHAN. ch. g., strip in face, white near hind ankle, foaled May 5, 1871; got by Abdallah Star, son of American Star, Jr., by Seely's American Star.

First Dam, Lady Rockafellow, by Seely's American Star.

Second Dam, by Bay Richmond.

Third Dam, by Gilbert Oliver's Jackson, by son of imp. Messenger.

American Star (Seely's), by Stockholm's American Star, son of Duroc.

First Dam, Sally Slouch, by Sir Henry.

Second Dam, by imp. Messenger.

Bay Richmond, by Toby, son of imp. Brown Highlander.

Dam, Lady Pluck, by Mambrino, son of imp. Messenger.

No. 5 .

GLENDENNING. ch. g., star and snip, white fore ankles, white near hind ankle, foaled May 28th, 1871; got by Messenger Duroc, son of Rysdyk's Hambletonian.

Dam, Glencoe Maid.

No. 6.

MARATHON. b. g., white near fore foot, white hind ankles, foaled June 5, 1871 ; got by Messenger Duroc, son of Rysdyk's Hambletonian.

Dam, Lucy Almack, by Young Engineer, son of Engineer 2d (sire of Lady Suffolk), by Engineer, son of imp. Messenger. 
No. 7.

ROCKET. ch. g., white hind ankles, foaled June 17, 1s71; got by Messenger Duroc, son of Rysdyk's Hambletonian. First Dam, Susie Roberts, by N. Y. Black Hawk.

Second Dam, the well known mare of Messenger descent, owned and driven by Capt. Roberts, of N. Y.

Black Hawk (New York), by L. I. Black Hawk.

First Dam, sister to Jack Cade, by Leopold (Arabian). Second Dam, by Bishop's Hambletonian, son of imp. Messenger.

No. 8.

CADMUS. b. g., star and snip, white hind heels, foaled July 16th, 1871 ; got by Abdallah Star, son of American Star, Jr., by Seely's American Star. First Dam, Alice Van Duzer, by Seely's American Star. Second Dam, by Messenger Duroc, son of Sir Archy Duroc.

Third Dam, by Paymaster, son of imp. Brown Highlander.

\section{TWO YEARS OLD GELDINGS.}

No. 1.

MANDARIN. b. g., star, white hind heels, foaled April 30, 1872 ; got by Idol, son of Rysdyk's Hambletonian.

First Dam, Trustus Webb, by Seely's American Star. Second Dam, by imp. Trustee. 
No. 2.

PROCTOR. b. g., star, white near hind heel, foaled May 3, 1872 ; got by Abdallah Star, son of American Star, Jr., by Seely's American Star.

First Dam, Bonnie Lassie, by Rysdyk's Hambletonian. Second Dam, by Fox Hunter.

No. 3.

MARQUIS. b. g., star, white near hind ankle, foaled May 26, 1872 ; got by Idol, son of Rysdyk's Hambletonian.

First Dam, Belle of Richmond, by Rysdyk's Hambletonian.

Second Dam, old Belle of Richmond, by Bathgate's Grey Norman.

No. 4.

EVANDER. b. g., star and snip, white near hind heel, foaled June 25, 1872; got by Idol, son of Rysdyk's Hambletonian.

First Dam, Alice Van Duzer, by Seely's American Star. Second Dam, by Messenger Duroc, son of Sir Archy Duroc.

Third Dam, by Paymaster, son of imp. Brown Highlander.

No. 5.

SATYR. b. g., black points, foaled August 14, 1872; got by Waverley, son of Rysdyk's Hambletonian.

First Dam, Lady Pierson, by Neave's Cassius M. Clay, Jr., son of Cassius M. Clay.

Second Dam, by Diamond, son of American Eclipse. 


\section{BROOD MARES.}

No. 1.

ALICE VAN DUZER. blk. mare, 15 hands, strip in face, white hind ankles, white near fore heel, foaled 1852 ; got by Seely's American Star.

First Dam, by Messenger Duroc.

Second Dam, by Paymaster.

American Star (Seely's), by Stockholm's American Star, son of Duroc.

First Dam, Sally Slouch, by Sir Henry.

Second Dam, by imp. Messenger.

Messenger Duroc, by Sir Archy Duroc, son of Duroc.

Dam, by Messenger, son of imp. Messenger.

Paymaster, by imp. Brown Highlander.

First Dam, by imp. Highflyer.

Second Dam, by Rainbow, son of imp. Wildair.

Third Dam, by True Briton.

1870. h. c. Legate, gelded .... Pierson.

1871. b. c. Cadmus, gelded ... Abdallah Star.

1872. b. c. Evander, gelded .. Idol.

1873. blk. c.............. Messenger Duroc. 
No. 2.

LADY BROWN. br. m., 15 hands, star and snip, white off hind Dam of Gauntlet and heel, foaled 1861; got by Seely's American Star.
Wilkins Micawber.

First Dam, by Nigger Lance, son of Lance.

American Star (Seely's), by Stockholm's American Star, son of Duroc.

First Dam, Sally Slouch, by Sir Henry.

Second Dam, by imp. Messenger.

Lance, by American Eclipse, son of Duroc.

Dam, the Dam of Ariel, by Financier.

1867. br. c. Gauntlet, gelded...Hambletonian. 1868. br. c. Wilkins Micawber...Hambletonian. 1869. br. c. Pickwick, gelded ... Idol. 1870 . Barren ..................

1871. b. c. Dickens, gelded.... Messenger Duroc. 1872. br. f. Mary Grafton...... Hambletonian. 1873. Barren

No. 3.

LUCY ALMACK. b. m. 15.2 hands, star, white off hind ankle, white near fore heel, foaled 1852; got by Young Engineer, son of Engineer 2d (sire of Lady Suffolk), by Engineer, son of imp. Messenger.

1867. b. f. Josephine ........ Hambletonian. 1868. Barren

1869. b. c. Arlington ........ Idol.

1870. b. f. Lucetta .......... Hambletonian.

1871. b. c. Marathon, gelded...Messenger Duroc. 1872. br. c. Antenor............ Messenger Duroc. 
No. 4.

MAGGIE JONES. ch.m., 15.1 hands, star, little white on near hind heel, foaled 1852; got by Seely's American Star.

Dam, by Mambrino Chief, son of Mambrino Paymaster. American Star (Seely's), by Stockholm's American Star, son of Duroc.

First Dam, Sally Slouch, by Sir Henry. Second Dam, by imp. Messenger.

1868. b. c. Romulus........ Hambletonian. 1869. ch. f. Mignonnette..... Idol.

1570. b. c. Major Morton.... Hambletonian.

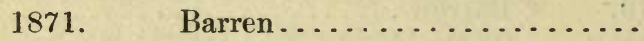
187\%. b. c. Mohican ........ Hambletonian. 1873. b. f. ............. Hambletonian. 
No. 5 .

ANNIE SEELY. b. m., $15.1 \frac{1}{2}$ hands, black points, foaled 1858; got by General Gifford, son of Gifford Morgan, by Woodbury Morgan.

First Dam, Seely Abdallah, by Abdallah, son of Mambrino.

Second Dam, Belle, by Webber's Tom Thumb.

Third Dam, Charles Kent Mare (Dam of Rysdyk's Hambletonian). By imp. Bellfounder.

Fourth Dam, One Eye, by Bisbop's Hambletonian.

Fifth Dam, Silvertail, by imp. Messenger.

Abdallah, by Mambrino, son of imp. Messenger.

Dam, Amazonia, by son of imp. Messenger.

Hambletonian (Bishop's), by imp. Messenger.

First Dam, Pheasant, by imp. Shark.

Second Dam, by imp. Medley.

1866. br. c. Stony Ford ........ Hambletonian.

1867. b. f. Donna... Fitzgerald-Hambletonian.

1868. b. f. Zerlina ............ Hambletonian.

1869. ch. c. Redgauntlet........ Idol.

1870.

Barren .....................

1871.

Barren

1872.

Barren .

1873. Not stinted for 1873 . 
No. 6.

LADY DURLAND. ch. m., 15 hands, star and snip, foaled 1853 ; got by Seely's American Star.

Dam, by Nigger Lance, son of Lance.

American Star (Seely's), by Stockholm's American Star, son of Duroc.

First Dam, Sally Slouch, by Sir Henry.

Second Dam, by imp. Messenger.

Lance, by American Eclipse, son of Duroc.

First Dam, Dam of Ariel, by Financier.

Second Dam, Empress, by imp. Baronet.

1866. b. c. Island Boy, gelded... Hetzell's Hambletonian. 1869. ch. f. . . . . . . . . . . . . . Idol. 1870.

Barren ...............

1871. b. f. Glenrose .......... Idol.

1872.

Barren ...............

1873.

Barren 
No. 7. .

BEIJLE OF RICHMOND. gr. m., 15.1 hands, white near hind ankle, foaled 1861; got by Rysdyk's Hambletonian.

Dam, Belle of Richmond, by Bathgate's Grey Norman. Norman (Bathgate's), by Morse Horse.

Dam, by Bishop's Hambletonian.

Hambletonian (Bishop's), by imp. Messenger.

First Dam, Pheasant, by imp. Shark.

Second Dam, by imp. Medley.

1868. gr. c. Contraband .......... War Eagle.

1869. br. c. Twinkle ............ Idol.

1870. Premature...............

1871. b. c. .................. Idol.

1872. b. c. Marquis ............ Idol.

1873. Barren

No. 8.

LADY PACKER. gr. m., 15.2 hands, black points, foaled 1860 ; got by Witherell's Messenger, son of Winthrop Messenger, by imp. Messenger.

1869. b. f. Sunbeam......... Idol.

1870.

Barren

1871. b. c. Dazzle, gelded ... Messenger Duroc. 1872. ch. f. Doretta ......... Messenger Duroc. 1873. b. f. .............. Messenger Duroc. 
No. 9.

MISTRESS HENYON. ch. m., 15.2 hands, star and strip, white hind legs, white near fore heel, foaled 1857 ; got by Roe's Abdallah Chief.

Dam, by Sir Henry.

Abdallah Chief (Roe's), by Abdallah, son of Mambrino.

First Dam, by Philips, son of Duroc.

Second Dam, by Decatur, son of Sir Henry.

Sir Henry, by Sir Archy, son of imp. Diomed.

First Dam, by imp. Diomed.

Second Dam, Bellona, by Bel-Air, son of imp. Medley.

1867. b. e. Argyle ................ Young Guy Miller.

1868. Premature foal.................

1869. b. f. Evelina .................. Idol.

1870. Barren .....................

1871. ch.f. Elinor................... Abdallah Star.

1872. b. c. Ferdinand, died March, 1873.... Idol.

1873. eh. e...................... Idol.

No. 10.

MILDRED. b. m., 15 hands, star, white off hind ankle, foaled 1858; got by Rysdyk's Hambletonian.

Dam, Linda (the old trotting mare), by Abel Horse, son of Smith's Napoleon, by Napoleon.

Napoleon, by Young Mambrino.

Dam, by American Commander.

Young Mambrino, by Chancellor, son of Mambrino, by imp. Messenger.

Dam, by Duroc, son of imp. Diomed.

American Commander, by Commander, son of imp. Messenger.

Dam, by imp. Light Infantry.

1869. b. f. Dead ........... Messenger Duroc.

1870. br. f. Emma Frances... Messenger Duroc.

1871. b. c. Waldo .......... Messenger Duroc.

1872. Foal strangled..............

1873. b. c. . . . . . . . . . Boltnu. 
No. 11.

GREEN MOUNTAIN MAID. br. m., 15 hands, star,

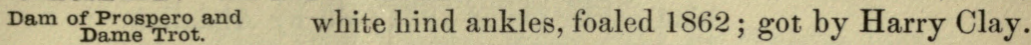
Harry Clay, by Neave's Cassius M. Clay, Jr.

Dam, by imp. Bellfounder.

Cassius M. Clay, Jr. (Neave's), by Cassius M. Clay, son of Henry Clay, by Andrew Jackson.

First Dam, by Chancellor, son of Mambrino.

- Second Dam, by Engineer 2d (sire of Lady Suffolk), by Engineer.

Engineer 2d, by Engineer, son of imp. Messenger.

First Dảm, by Plato.

Second Dam, by Rainbow.

Plato, by imp. Messenger.

First Dam, Pheasant, by imp. Shark.

Second Dam, by imp. Medley.

Rainbow, by imp. Wildair.

First Dam, by General Heard's True Briton.

Second Dam, an imp. mare, by Duke of Bolton's Starling.

1867. b. f. Storm............... Middletown.

1868. b. c. Electioneer .......... Hambletonian.

1869. blk. c. Prospero, gelded ...... Messenger Duroc. 1870. blk. f. Dame Trot .... . . . . Messenger Duroc. 1871. ch. c. Paul, gelded ...........Messenger Duroc. 1872. ch. f. Miranda.............. Messenger Duroc. 1873. blk. c. (killed Sept. 15, 1873)...Messenger Duroc. 
No. 12.

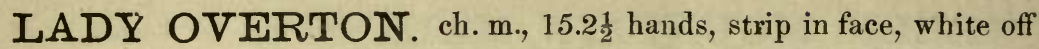
hind ankle, foaled 1851; got by Abdallah.

Dam, by Mount Holly.

Abdallah, by Mambrino, son of imp. Messenger.

Dam, Amazonia, by son of imp. Messenger.

Mount Holly, by imp. Messenger.

First Dam, by Bajazet, son of imp. Tanner.

Second Dam, by Bashaw, son of imp. Wildair.

1867. ch. c. Richard 1st, gelded.. Woodpecker.

1868. b. f. Queen of May ........ Hambletonian.

1869. ch. c. Gardner, gelded ...... Messenger Duroc.

1870.

Barren ......................

1871. ch. f. Laurel ............ Messenger Duroc.

1872. ch. c. Ovation .......... Idol.

1873. b. f.

Messenger Duroc.

No. 13.

NELIIE WILMARTH. br. m., 15 hands, star, white hind heels, foaled June, 1855; got by Raven.

First Dam, Jennie Lind, by young Andrew Jackson.

Second Dam, Polly Sherman, by Bush's Messenger, son of imp. Messenger.

Raven, by Hill's Vermont Black Hawk, son of Sherman Morgan.

'Dam, by Woodbury Morgan, son of Justin Morgan. Andrew Jackson (Young), by Andrew Jackson, son of Young Bashaw, by Grand Bashaw.

Dam, Mambrino mare, Great Western.

1869. ch. c. Wilmarth ........ Idol.

1870.

Barren....................

1871. blk. c. Maclure, gelded...Messenger Duroc. 
No. 14.

VIRGO. blk. m., 15.1 hands, white near hind heel, foaled 1855; got by Abdallah Chief (Roe's).

First Dam, Catbird, foaled 1834, by Whistle Jacket.

Second Dam, Lyon Mare, by Bertholf Horse.

Third Dam, by Duroc, son of imp. Diomed. Abdallah Chief (Roe's), by Abdallah.

First Dam, by Philips, son of Duroc.

Second Dam, by Decatur, son of Sir Henry.

Abdallah, by Mambrino, son of imp. Messenger.

Dam, Amazonia, by son of imp. Messenger.

Whistle Jacket, by Mambrino, son of imp. Messenger.

First Dam, by American Eclipse, son of Duroc.

Second Dam, by Bertholf Horse.

Bertholf Horse, by imp. Messenger.

Dam, Beauchamp Mare, by Duroc, son of imp. Diomed.

1869. br. c. Ravenswood.... Messenger Duroc.

1870. b. c. Ellwood ........ Messenger Duroc.

1871. blk. f. Helen Russell .. Messenger Duroc.

1872. b. f. Belle Rose ..... Idol.

1873.

Barren

No. 15.

MARY HULSE. b. m., 15 hands, white hind ankles, foaled 1860 ; got by Seely's American Star.

Dam, of Messenger deseent.

American Star (Seely's), by Stockholm's American Star, son of Duroc.

First Dam, Sally Slouch, by Sir Henry.

Second Dam, by imp. Messenger.

1868. b. c. Twilight .......... Hambletonian.

1869. b. c. Rival ............... Middletown.

1870. b. f. Dead .............. Messenger Duroc.

1871. ch.f. Dawn ............ Messenger Duroc.

1872. b. c. King William ....... Hambletonian.

1873. b. f. .............. Hambletonian. 
No. 16.

FANNIE STAR. b. m., 15.2 hands, black points, foaled 1860 ; got by Seely's American Star.

Dam, Monnell Mare, by Abdallah.

American Star (Seely's), by Stockholm's American Star, son of Duroc.

First Dam, Sally Slouch, by Sir Henry.

Second Dam, by imp. Messenger.

Abdallah, by Mambrino, son of imp. Messenger.

Dam, Amazonia, by son of imp. Messenger.

1870. ch. c. ............. Messenger Duroc.

1871 .

Barren .................

1872. b. f. Glenmary ...... Hambletonian.

1873. b. f.

. Hambletonian.

No. 17 .

TRUSTUS WEBB. b. m., 15.2 hands, black points, foaled 1860 ; got by Seely's American Star.

Dam, by imp. Trustee.

American Star (Seely's), by Stockholm's American Star, son of Duroc.

First Dam, Sally Slouch, by Sir Henry.

Second Dam, by imp. Messenger.

Trustee (imp.), by Catton.

First Dam, Emma, by Whisker.

Second Dam, Gibside Fairy, by Hermes.

Third Dam, Vicissitude, by Pipator.

Fourth Dam, Beatrice, by Sir Peter.

Fifth Dam, Pyrrha, by Matchem.

Sixth Dam, Duchess, by Whitenose.

Seventh Dam, Miss Slamerkin, by Young T'rue Blue.

Eighth Dam, by Lord Oxford's Dun Arabian.

Ninth Dam, D'Arcy's black legged Royal Mare.

1870. b. c. Messenger Duroc, $\mathrm{J}_{\mathrm{r}}$........ Messenger Duroc.

1871. b. f. Christine (died Feb'y, 1873).. Messenger Duroc.

1872. b. c. Mandarin, gelded ......... Idol.

1873. b. c.

Idol. 
No. 18.

LADY NODINE. br. m., 15.1 hands, white hind heels, foaled 1863 ; got by Rysdyk's Hambletonian.

Dam, Montgomery Maid, by Seely's American Star.

American Star (Seely's), by Stockholm's American Star, son of Duroc.

First Dam, Sally Slouch, by Sir Henry.

Second Dam, by imp. Messenger.

1870. ch. c. Gleneida, gelded.. Messenger Duroc.

1871. b. f. Lorraine .......... Messenger Duroc.

1872. ch. f. Iona ........... Abdallah Star.

1873. ch. c. Spencer .......... Messenger Duroc.

No. 19.

LADY CARDINAL. blk. m., 15.1 hands, star and snip, white Dam of legs, foaled 1863; got by Harry Clay, son of Neave's Cassius M. Clay, Jr.

First Dam, by Cardinal.

Second Dam, Harry Clay's Dam, by imp. Bellfounder.

Cassius M. Clay, Jr. (Neave's), by Cassius M. Clay, son of Henry Clay, by Andrew Jackson.

First Dam, by Chancellor, son of Mambrino.

Second Dam, by Engineer 2d (sire of Lady Suffolk), by Engineer, son of imp. Messenger.

1870. b. c. Reform, gelded ............Messenger Duroc. 1871. b. f. Blooming ................ Messenger Duroc. 1872. b. c. Ruskin (died Feb., 1873)...Messenger Duroc. 1873. blk. c. ..................... Messenger Duroc. 
No. 20.

MATTIE. b. m., 15.2 hands, black points, foaled 1866; got by Rysdyk's Hambletonian.

First Dam, Lady Earle, by Chauncey Green.

Second Dam, by Judson's Hambletonian.

Third Dam, by Harris' Hambletonian.

Fourth Dam, by Judson's Hambletonian.

Fifth Dam, by Harris' Hambletonian.

Chauncey Green, by Hale's Green Mountain Morgan.

Dam, by Judson's Hambletonian.

Green Mountain Morgan (Hale's), by Gifford Morgan, son of Woodbury Morgan.

Dam, by Woodbury Morgan.

Hambletonian (Judson's), by Bishop's Hambletonian, son of imp. Messenger.

Dam, by Wells' Magnum Bonum.

Hambletonian (Harris'), by Bishop's Hambletonian, son of imp. Messenger.

Dam, the Mare owned by Israel Munson, of Boston, and represented to be by imp. Messenger.

1870. b. c. Elander H. Sherman......... Idol.

1871. Not stinted for 1871 .

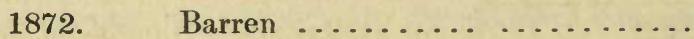

1873. ch. c. .................. Idol.

No. 21 .

BONNIE LASSIE. b. m., $15.1 \frac{1}{2}$ hands, star and snip, white near hind heel, foaled 1865; got by Rysdyk's Hambletonian.

Dam, by Fox Hunter.

1870. b. f. Finesse............ Pierson.

1871.

Not stinted for 1871.

1872. b. c. Proctor, gelded ....... Abdallah Star.

1873.

Barren...................... 
No. 22.

AMANDA. b. m., 15.3 hands, star and snip, little white on off hind heel, foaled 1866; got by Rysdyk's Hambletonian. First Dam, by Cardinal.

Second Dam, Rhoda, by One-Eyed Kentucky Hunter.

1870. b. f. Lulu ................. Pierson.

Not stinted for 1871, 1872, 1873.

No. 23.

LADY DEXTER. b. m., 15 hands, strip in face, white legs, foaled Full Sister 1861 ; got by Rysdyk's Hambletonian.

Dam, Dexter's Dam, by Seely's American Star. Ameriean Star (Seely's), by Stockholm's American Star, son of Duroc.

First Dam, Sally Slouch, by Sir Henry.

Second Dam, by imp. Messenger.

1868. br. c. Briney ......... Mambrino Pilot.

1871. b. f. Dexterity, dead ...Messenger Duroc.

1872. blk. c. Victor Duroc.....Messenger Duroc.

1873. Barren ..................... 
No. 24.

EMMA MILLS. ch. m., 15.1 hands, strip in face, white near hind Dam of heel, foaled 1853; got by Seely's American Star.

Dam, by Radiker's Alexander W., son of Alexander, thoroughbred from Virginia.

American Star (Seely's), by Stockholm's American Star, son of Duroc.

First Dam, Sally Slouch, by Sir Henry.

Second Dam, by imp. Messenger.

1865. b. f. Fairy .......... Hambletonian.

1869. b. f. Diana ......... Pahallas.

1870.

Barren

1871. ch. c. Glenair ......... Messenger Duroc.

1872.

Premature ................

1873. b. f.

Hambletonian.

No. 25.

GLENCOE MAID. ch. m., 15.1 hands, strip in face, near white hind ankle, foaled 1855.

1871. ch. c. Glendenning, gelded....Messenger Duroc. 1872. Premature

1873. Barren 
No. 26.

LADY FALLIS. b. m., 15.3 hands, strip in face, foaled 1859; got by Seely's American Star.

Dam by L. I. Black Hawk.

American Star (Seely's), by Stockholm's American Star, son of Duroc.

First Dam, Sally Slouch, by Sir Henry.

Second Dam, by imp. Messenger.

Black Hawk (Long Island), by Andrew Jackson, son of Young Bashaw, by Grand Bashaw.

Dam, Sally Miller, by Mambrino, son of imp. Messenger.

1865. b. f. Sally Mills .............. Major Winfield.

1866. b. c. Socrates .............. Hambletonian.

1867. b. c. Iago ................... Middletown.

1868. b. c. Norwood .............. Hambletonian.

1869. b. f. Gretchen .............. Chosrosse.

1870. b. c. Tatternack .............. Mills' Hambletonian.

1871. b. f. Xantippe .............. Hambletonian.

1872. b. c. Pickering ............. Hambletonian.

1873. b. c. .................... Hambletonian.

No. 27.

NELLIE SAYRE. br.m., 15.1 hands, star, white off hind heel, foaled 1853; got by Seely's American Star.

First Dam, by Hulse's Hickory.

Second Dam, by La Tourett's Bellfounder.

American Star (Seely's), by Stockholm's American Star, son of Duroc.

First Dam, Sally Slouch, by Sir Henry.

Second Dam, by imp. Messenger.

1870. br. f. Constance......... Knickerbocker.

1871.

Barren ....................

1872. br. f. Rosalinda ........ Hambletonian.

1873. br. c. Dean Sage ........ Hambletonian. 
No. 28.

EMMA HOYT. ch. m., 15.2 hands, star and snip, white near hind heel, foaled 1859; got by Seely's American Star.

First Dam, by Ohio Eclipse, son of American Eclipse.

Second Dam, a mare of Messenger descent.

American Star (Seely's), by Stockholm's American Star, son of Duroc.

First Dam, Sally Slouch, by Sir Henry.

Second Dam, by imp. Messenger.

1870. b. c. ................. Conductor.

1871. ch. c. Quartette ............ Idol.

1872. ch. f. Quarto .............. Idol.

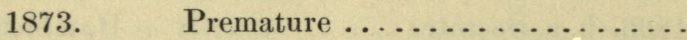

No. 29.

HATTIE HOGAN. br. m., 15.3 hands, star and snip, white hind heels, foaled 1867; got by Harry Clay.

First Dam, Nellie Sayre, by Seely's American Star.

Second Dam, by Hulse's Hickory.

Third Dam, by La Tourett's Bellfounder.

Harry Clay, by Neave's Cassius M. Clay, Jr.

Dam, by imp Bellfounder.

Cassius M. Clay, Jr. (Neave's), by Cassius M. Clay.

First Dam, by Chancellor, son of Mambrino.

Second Dam, by Engineer 2d (sire of Lady Suffolk), by Engineer, son of imp. Messenger.

American Star (Seely's), by Stockholm's American Star, son, of Duroc.

First Dam, Sally Slouch, by Sir Henry.

Second Dam, by imp. Messenger.

187\%. b. c. Marengo ........ Messenger Duroc.

1873. blk. c. Hogarth ........ Messenger Duroc. 
No. 30 .

ADELE. b. m., 15.2 hands, star and snip, white hind ankles, white near fore heel, foaled 1867; got by Rysdyk's Hambletonian.

Dam, Lady Franklin (owned by David Bonner, Esq.)

1872. ch. c. .............. Abdallah Star.

1873. ch. f. .............. Messenger Duroc.

No. 31.

JANE MURRAY. gr. m., 15.1 hands.

Dam of Lottery.

No. 32.

KITTIE HARKER. b. m., 15.2 hands, strip in face, white off hind heel, white spot on off fore heel, foaled 1863; got by Ethan Allen, son of Hill's Vermont Black Hawk. First Dam, Martha, by Abdallah.

Second Dam, by Young Bellfounder.

Third Dam, by Corn Cracker.

Fourth Dam, by Hickory.

Abdallah, by Mambrino, son of imp. Messenger.

Dam, Amazonia, by son of imp. Messenger.

Bellfounder (Young), by imp. Bellfounder,

Dam, by Wildair, son of Decatur.

1872. b. f. Adriana.......... Messenger Duroc. 1873. b. c. ................ Messenger Duroc. 
No. 33.

FAIRY. b. m., 15.2 hands, white hind ankles, foaled 1865 ; got by Rysdyk's Hambletonian.

First Dam, Emma Mills, by Seely's American Star.

Second Dam, by Radiker's Alexander W., son of Alexander (thoroughbred from Virginia).

American Star (Seely's), by Stockholm's American Star, son of Duroc.

First Dam, Sally Slouch, by Sir Henry.

Second Dam, by imp. Messenger.

1872. b. f. Clarabel .......... Abdallah Star.

1873. ch. f. ............... Abdallah Star.

No. 34.

BLOSSOM. b. m., 15.2 hands, small star, white near hind heel, foaled May 1, 1868; got by Rysdyk's Hambletonian.

First Dam, Hattie Woud, by Harry Clay.

Second Dam, Grandmother, by 'Terror.

Harry Clay, by Neave's Cassius M. Clay, Jr.

Dan, by imp. Bellfounder.

Cassius M. Clay, Jr. (Neave's), by Cassius M. Clay, son of Henry Clay, by Andrew Jackson, son of Young Bashaw, by Grand Bashaw.

First Dam, by Chancellor, son of Mambrino.

Second Dam, by Engineer 2d, (sire of Lady Suffolk).

Son of Engineer, by imp. Messenger.

Terror, by American Eclipse.

First Dam, Lady Lightfoot, by Sir Archy.

Second Dam, Black Maria, by imp. Shark.

Third Dam, by imp. Clockfast.

Fourth Dam, Burwell's Maria, by Regulus.

Fifth Dam, Camilla, by imp. Fearnaught.

Sixth Dam, Calesta, by Forrester. 
No. 35 .

LADY TALMADGE. ch. m., 15.1 hands, strip in face, white off hind ankle, foaled 1853; got by Seely's American Star.

Dam, from Western New York.

American Star (Seely's), by Stockholm's American Star, son of Duroc.

First Dam, Sally Slouch, by Sir Henry.

Second Dam, by imp. Messenger.

1871. b. f. Ulema ........... Conductor.

1872. b. c. Pericles .......... Hambletonian.

1873. b. f. ............... Hambletonian.

No. 36.

MARY SANDFORD. b. m., 15 hands, white near heels, foaled 1862 ; got by Plunket Horse.

Dam, Lady Sanford (Dam of Jay Gould), by Seely's American Star.

Plunket Horse, by Alexander's Abdallah.

Dam, by Bay Richmond.

Abdallah (Alexander's), by Rysdyk's Hambletonian.

First Dam Katy Darling, by Bay Roman.

Second Dam, by Mambrino, son of imp. Messenger.

Bay Richmond, by Toby, son of imp. Brown Highlander.

Dam, Lady Pluck, by Mambrino.

American Star (Seely's), by Stockholm's American Star, son of Duroc.

First Dam, Sally Slouch, by Sir Henry.

Second Dam, by imp. Messenger.

1871. b. c. ................... Imperial.

1872. b. c. ................... Imperial. 
No. 37.

FANNY HARRIS. ch. m., 15.2 hands, small star in forehead, foaled 1853; got by Seely's American Star.

First Dam, by Black Rock, son of Roebuck.

Second Dam, by Walden Messenger.

Third Dam, foaled 1798, by imp. Messenger.

American Star (Seely's), by Stockholm's American Star, son of Duroc.

First Dam, Sally Slouch, by Sir Henry.

Second Dam, by imp. Messenger.

1872. b. f. Lucia ............... Speedwell.

1873.

Barren .. 
No. 38.

ANTOINETTE. b. m., 15.2 hands, star, white off hind leg, foaled 1864 ; got by Shepherd's Rattler.

First Dam, by Young Bulle Rock, son of Edward Long's Eclipse.

Second Dam, by Edward Long's Henry 2d.

Rattler (Shepherd's), by Biggart's Rattler.

Dam, by Edward Long's Tornado.

Rattler (Biggart's), by Sir Henry, son of the Garret Horse.

Dam, by Hyde Horse, son of Edward Long's Magnum Bonum.

Tornado (Long's) by American Eclipse, son of Duroc.

First Dam, Polly Hopkins, by Virginian.

Second Dam, Janey, by imp. Archduke.

Third Dam, by imp. Stirling.

Fourth Dam, by'imp. Obscurity.

Fifth Dam, Miss Slamerkin, by imp. Wildair.

Eclipse (Long's), by American Eclipse, son of Duroc.

First Dam, by Bajazet, son of imp. Tanner.

Second Dam, by Mercury, son of imp. Janus.

Third Dam, by imp. Messenger.

Fourth Dam, by Badger, son of imp. Badger.

Henry 2d (Long's), by Sir Henry, son of Sir Archy.

First Dam, the Dam of Sir Lovel, by imp. Light Infantry.

Second Dam, by imp. Messenger.

Third Dam, by Bashaw, son of imp. Wildair.

Fourth Dam, by True Briton, son of imp. Othello.

Fifth Dam, by imp. Sterling. 
No. 39.

GLENELLA. ch. m., 15.2 hands, strip in face, white hind legs, foaled 1864 ; got by Woodward Star, son of Seely's American Star.

Dam, the Dam of Green Mountain Maid.

American Star (Seely's), by Stockholm's American Star, son of Duroc.

First Dam, Sally Slouch, by Sir Henry.

Second Dam, by imp. Messenger.

1871. b. f. ................ Imperial.

1872. ch. c. ................. Imperial.

1873.

Barren ...................

No. 40.

ROSETTA. b. m., 15.1 hands, little white on hind heels, foaled April 22d, 1867; got by Rysdyk's Hambletonian.

Dam, Grey Rose, by Harris' Hambletonian.

Hambletonian (Harris'), by Bishop's Hambletonian.

Dam, the mare owned by Israel Munson, of Boston, and represented to be by imp. Messenger.

Hambletonian (Bishop's), by imp. Messenger.

First Dam, Pheasant, by imp. Shark.

Second Dam, by imp. Medley. 
No. 41.

MINEOLA. b. m., 15.2 hands, star in forehead, spot on nose, white near hind ankle, foaled May, 1868; got by Rysdyk's Hambletonian.

First Dam, Lady. Banker, by Roe's Abdallah Chief.

Second Dam, by Saltram.

Abdallah Chief (Roe's), by Abdallah, son of Mambrino.

First Dam, by Philips, son of Duroc.

Second Dam, by Decatur, son of Sir Henry.

Saltram, by Webber's Kentucky Whip, son of Blackburn's Whip.

First Dam, by Sea Gull, son of Duroc.

Second Dam, by Camptown, son of Leopold.

Whip (Blackburn's), by imp. Whip.

First Dam, Speckleback, by Celer.

Second Dam, by Pilgrim.

Third Dam, by imp. Janus.

Fourth Dam, by imp. Valiant.

Fifth Dam, by imp. Jolly Roger.

Sixth Dam, by imp. Shock.

Seventh Dam, by imp. Sober John.

Leopold, by Ogle's Oscar, son of imp. Gabriel.

First Dam, Katydid, by imp. Expedition.

Second Dam, by imp. Sourcrout.

Third Dam, Matchless, by imp. slender.

Fourth Dam, Fair American, by Traveller.

Fifth Dam, Miss Slamerkin, by imp. Wildair.

No. 42.

QUEEN OF MAY. b. m., 15.2 hands, white near hind ankle, white off hind heel, foaled May 1, 1868; got by Rysdyk's Hambletonian.

First Dam, Lady Overton, by Abdallah.

Second Dam, by Mount Holly.

Abdallah, by Mambrino, son of imp. Messenger.

Dam, Amazonia, by son of imp. Messenger.

Mount Holly, by imp. Messenger.

First Dam, by Bajazet, son of imp. Tanner.

Second Dam, by Bashaw, son of imp. Wildair. 
No. 43.

CAROL br. m., 15.2 hands, white off fore ankle, white off hind heel, foaled August 3, 1870; got by Waverley, son of Rysdyk's Hambletonian.

Dam, Lady Farr, by Abdallah.

Abdallah, by Mambrino, son of imp. Messenger.

Dam, Amazonia, by son of imp. Messenger.

No. 44.

LAVINIA. br.m., 15.1 hands, black points, foaled 1868; got by Truesdell's Hambletonian.

First Dam, Babbitt Abdallah (sister of Roe's Abdallah Chief), by Abdallah.

Second Dam, by Philips, son of Duroc.

Third Dam, by Decatur, sou of Sir Henry.

Hambletonian (Truesdell's), by Welling Colt (owned by John

Linn, Newton N. J.), son of Rysdyk's Hambletonian.

First Dam, Juda, by Liberty.

Second Dam, by imp. Bellfounder.

Liberty, by Lance, son of American Eclipse.

First Dam, Matchless, by imp. Expedition.

Second Dam, Spiletta, by Sir Solomon.

Third Dam, Aurora, by inip. Honest John.

Fourth Dam, Zelipha, by imp. Messenger.

Abdallah, by Mambrino, son of imp. Messenger.

Dam, Amazonia, by son of imp. Messenger.

No. 45 .

STARBEAM. b. m., 15.1 hands, star, white on lip, white near fore ankle, white hind ankles, foaled June 12, 1868; got by Rysdyk's Hambletonian.

Dam, Montgomery Maid, by Seely's American Star.

American Star (Seely's), by Stockholm's American Star, sou of Duroc.

First Dam, Sally Slouch, by Sir Henry, son of Sir Archy. Second dam, by imp. Messenger. 
No. 46.

ARIEL. b. m., 15.2 hands, star and snip, white hind feet, foaled - ; got by Ethan Allen, son of Hill's Vermont Black Hawk.

Dam, by Harris' Hambletonian.

Hambletonian (Harris') by Bishop's Hambletonian.

Dam, the mare owned by Israel Munson, of Boston, and represented to be by imp. Messenger.

Hambletonian (Bishop's), by imp. Messenger.

First Dam, Pheasant, by imp. Shark.

Second Dam, by imp. Medley.

CHARIM. b. m., $15.2 \frac{1}{2}$ hands, star and snip, white hind ankles, foaled May 1, 1870 ; got by Idol, son of Rysdyk's Hambletonian.

First Dam, Satinet (Dam of Messenger Duroc), by Roe's Abdallah Chief.

Second Dam, Catbird, by Whistle Jacket.

Third Dam, Lyon Mare, by Bertholf Horse.

Abdallah Chief (Roe's), by Abdallah.

First Dam, by Philips, son of Duroc.

Second Dam, by Decatur, son of Sir Henry, by Sir Archy.

Abdallah, by Mambrino, son of imp. Messenger.

Dam, Amazonia, by son of imp. Messenger.

Whistle Jacket, by Mambrino, son of imp. Messenger.

First Dam, by American Eclipse, son of Duroc.

Second Dam, by Bertholf Horse.

Bertholf Horse, by imp. Messenger.

Dam, Beauchamp Mare, by Duroc, son of imp. Diomed. 
No. 48.

LOUIE. br. m., 16 hands, star, white off feet, foaled 1865 ; got by Rysdyk's Hambletonian.

First Dam, by Mambrino Chief, son of Mambrino Paymaster.

Second Dam, by Dunkin's Mambrino Messenger.

Mambrino Paymaster, by Mambrino, son of imp. Messenger.

Dam, by imp. Paymaster.

Mambrino Messenger (Dunkin's), by Mambrino Paymaster.

Dam, Lady Messenger, by Coffin's Messenger.

Messenger (Coffin's), by imp. Messenger.

Dam, by Feather, son of imp. Light Infantry.

No. 49.

BESS. b. m., 15.1 hands, star and snip, white near ankles, brown spot behind shoulder, foaled August 16, 1867 ; got by Rysdyk's Hambletonian.

First Dam, Jessie Sayre. by Harry Clay.

Second Dam, by Liberty.

Harry Clay, by Neave's Cassius M. Clay, Jr.

Dam, by imp. Bellfounder.

Cassius M. Clay, Jr. (Neave's), by Cassius M. Clay, son of Henry Clay, by Andrew Jackson.

First Dam, by Chancellor, son of Mambrino.

Second Dam, by Engineer 2d, son of Engineer, by imp. Messenger.

Liberty, by Lance, son of American Eclipse.

First Dam, Matchless, by imp. Expedition.

Second Dam, Spiletta, by Sir Solomon.

Third Dam, Aurora, by imp. Honest John.

Fourth Dam, Zelipha, by imp. Messenger. ankles, foaled May 7, 1870; got by Rysdyk's Hambletonian.

Dam, Kate (Dam of Brunctte and Bruno). 
No. 1.

\section{SEVEN YEARS OLD MARES.}

RACOLA. br. m., white hind heels, foaled April 22d, 1867 ; got by Rysdyk's Hambletonian.

Dam, Brown Pacer.

No.2.

JOSEPHINE. b. m., star, white hind ankles, foaled August 13th, 1867 ; got by Rysdyk's Hambletonian.

Dam, Lucy Almack, by Young Engineer, son of Engineer 2d (sire of Lady Suffolk), by Engineer, son of imp. Messenger.

\section{SIX YEARS OLD MARES.}

No. 1.

UNDINE. b. m., white near hind ankle, foaled June 26, 1868; got by Rysdyk's Hambletonian.

First dam, Roan Marie.

No. 2.

MABI.E.b. m., star, white fore heels, white near hind ankle, white off hind heel,foaled October, 1868; got by Rysdyk's Hambletonian.

Dam, Lady Franklin, (owned by David Bonner, Esq.) 


\section{FIVE YEARS OLD MARE.}

SUNBEAM. b. m., white near hind heel, foaled June 20, 1869 ; got by Idol, son of Rysdyk's Hambletonian.

Dam, Lady Packer, by Witherell Messenger, son of Winthrop Messenger, by imp. Messenger.

\section{FOUR YEARS OLD MARES.}

No. 1.

LUCETTA. b. m., white hind ankles, white near fore heel, foaled May 6th, 1870 ; got by Rysdyk's Hambletonian.

Dam, Lucy Almack, by Young Engineer, son of Engineer 2d (sire of Lady Suffolk).

No. 2.

EMMA FRANCES. br. m., white off hind heel, foaled May 26, 1870 ; got by Messenger Duroc, son of Rysdyk's Hambletonian.

First Dam, Mildred, by Rysdyk's Hambletonian.

Second Dam, Linda (the old trotting mare), by Abel

Horse, son of Smith's Napoleon, by Napoleon, son of Young Mambrino, by Chancellor, son of Mambrino, by imp. Messenger.

No. 3.

ch. f., star in forehead, foaled 1870, got by Autocrat. Dam, by Otsego.

Autocrat, by Volunteer, son of Rysdyk's Hambletonian.

Dam, by Seely's American Star. 


\section{THREE YEARS OLD FILLIES.}

No. 1.

CARESS. b. f., star in forehead, spot on nose, white hind ankles, foaled April 11, 1871, got by Abdallah Star, son of American Star, Jr.

First Dam, Lady Bryant, by Brignoli, son of Mambrino Chief.

Second Dam, by Cracker, son of Boston (sire of Lexington).

No. 2.

XANTIPPE. b. f., star and snip, white hind heels, foaled April 15, 1871, got by Rysdyk's Hambletonian.

First Dam, Lady Fallis (Dam of Socrates), by Seely's American Star.

Second Dam, by Long Island Black Hawk, son of Andrew Jackson, by Young Bashaw.

No. 3.

ELINOR. ch. f., strip in face, white near hind ankle, white off hind heel, foaled May 11, 1871, got by Abdallah Star, son of American Star, Jr.

First Dam, Mistress Henyon, by Roe's Abdallah Chief, son of Abdallah, by Mambrino

Second Dam, by sir Henry, son of Sir Archy. 
No. 4.

GLENROSE. b. f., star and snip, white hind ankles, foaled May 25, 1871; got by Idol, son of Rysdyk's Hambletonian.

First Dam, Lady Durland, by Seely's American Star. Second Dam, by Nigger Lance, son of Lance, by American Eclipse.

No. 5.

LORRAINE. b. f., white hind heels, foaled May '26, 1871; got by Messenger Duroc, son of Rysdyk's Hambletonian. First Dam, Lady Nodine, by Rysdyk's Hambletonian. Second Dam, Montgomery Maid, by Seely's American Star.

No. 6.

BLOOMING. b.f., white face, white fore ankles, white near hind heel, foaled May 28th, 1871; got by Messenger Duroc, son of Rysdyk's Hambletonian.

First Dam, Lady Cardinal, by Harry Clay, son of Neave's Cassius M. Clay, Jr.

Second Dam, by Cardinal.

Third Dam, Harry Clay's Dam, by imp. Bellfounder.

No.7.

IDALIA. ch. f., white face, foaled May 29, 1871; got by Abdallah Star, son of American Star, Jr.

First Dam, Ledger Girl, by Rysdyk's Hambletonian.

Second Dam, by son of Barney's Henry, son of Edward Long's Henry 2d, by Sir Henry. 
No. 1.

\section{TWO YEARS OLD FILLIES.}

ROSALINDA. br. f., black points, foaled April 5th, 1872 ; got by Rysdyk's Hambletonian.

First Dam, Nellie Sayre, by Seely's American Star.

Second Dam, by Hulse's Hickory.

Third Dam, by La Tourett's Bellfounder.

No. 2.

ADRIANA. b. f., star, white hind ankles, foaled May 14th, 1872 ; got by Messenger Duroe, son of Rysdyk's Hambletonian.

First Dam, Kitty Harker, by Ethan Allen, son of Hill's Vermont Black Hawk.

Second Dam, Martha, by Abdallah, son of Mambrino. Third Dam, by Young Bellfounder, son of imp. Bellfounder.

Fourth Dam, by Corn Cracker.

Fifth Dam, by Hickory.

No.3.

GLENMARY. b. f., star, white hind ankles, foaled May 20th, 1872 ; got by Rysdyk's Hambletonian.

First dam, Fannie Star, by Seely's American Star.

Second Dam, Monnell Mare, by Abdallah, son of Mambrino. 
No. 4.

IOINA. ch. f., strip in face, white hind legs, white off fore heel, foaled May 22d, 1872; got by Abdallah Star, son of American Star, Jr.

First Dam, Lady Nodine, by Rysdyk's Hambletonian. Second Dam, Montgomery Maid, by Seely's American Star.

No. 5.

MIRANDA. ch. f., strip in face, white hind legs, foaled May 25th, 1872 ; got by Messenger Duroc, son of Rysdyk's Hambletonian.

First Dam, Green Mountain Maid (dam of Prospero), by Harry Clay, son of Neave's Cassius M. Clay, Jr.

No. 6.

CLARABEL. b. f., white off hind ankle, foaled May 30th, 1872 ; got by Abủallah Star, son of American Star, Jr. First Dam, Fairy, by Rysdyk's Hambletonian.

Second Dam, Emma Mills, by Seely's American Star. Third Dam, by Radiker's Alexander W., son of Alexander (thoroughbred from Virginia).

No. 7.

MARY GRAFTON. br. f., star and snip, white off fore ankle, white hind legs, foaled July i9th, 1872; got by Rysdyk's Hambletonian.

First Dam, Lady Brown, by Seely's American Star. Second Dam, by Nigger Lance, son of Lance, by American Eclipse. 


\section{YEARLING FILLIES.}

No. 1.

br. f., star, white hind heels, foaled April 21, 1873; got by Messenger Duroc, son of Rysdyk's Hambletonian.

First Dam, Nellie Wilmarth, by Raven, son of Hill's Vermont Black Hawk.

Second Dam, Jennie Lind, by Young Andrew Jackson, son of Andrew Jackson, by Young Bashaw.

Third Dam, by Bush's Messenger.

No. 2.

b. f., small star, white hind heels, foaled May 6, 1873; got by Rysdyk's Hambletonian. First Dam, Mary Hulse, by Seely's American Star. Second Dam, of Messenger descent.

No. 3.

b. f., small star, white near hind heel, white spot on off hind heel, foaled May 10, 1873; got by Rysdyk's Hambletonian.

First Dam, Fannie Star, by Seely's American Star.

Second Dam, Monnell Mare, by Abdallah, son of Mambrino. 
No. 4.

b. f., white hind heels, foaled May 13, 1873 ; got by Rysdyk's Hambletonian.

First Dam, Maggie Jones, by Seely's American Star. Second Dam, by Mambrino Chief.

No. 5 .

ch. f., blaze in face, white legs, foaled May 18, 1873; got by Messenger Duroc, son of Rysdyk's Hambletonian.

First Dam, Adele, by Rysdyk's Hambletonian. Second Dam, Lady Franklin.

No. 6.

ch. f., white hind feet, foaled May 22d, 1873 ; got. by Abdallah Star, son of American Star, Jr., by Seely's American Star.

First Dam, Fairy, by Rysdyk's Hambletonian.

Second Dam, Emma Mills, by Seely's American Star. Third Dam, by Radiker's Alexander W.

No. 7.

b. f., star, spot on nose, white near hind leg, white off hind foot, foaled June 9th, 1873; got by Messenger Duroc, son of Rysdyk's Hambletonian. Dam, Lady Packer, by Witherell Messenger, son of Winthrop Messenger, by imp. Messenger. 
No. 8.

b. f., white near fore heel, white hind ankles, foaled June 30, 1873; got by Messenger Duroc, son of Rysdyk's Hambletonian.

First Dam, Lady Overton, by Abdallah, son of Mambrino. Second Dam, by Mount Holly, son of imp. Messenger.

No. 9.

b. f., star, white hind legs, foaled August 15, 1873 ; got by Rysdyk's Hambletonian.

Dam, Lady Talmadge, by Seely's American Star.

No. 10.

b. f., star in forehead, black points, foaled August 30, 1873 ; got by Rysdyk's Hambletonian.

First Dam, Emma Mills, by Seely's American Star. Second Dam, by Radiker's Alexander W. 


\section{NOTICE.}

My Annual Sales will be held on the Second Tuesday in June of each succeeding year.

Charles Backman. 





\section{DAY USE \\ RETURN TO DESK FROM WHICH BORROWED}

\section{CIRCULATION DEPARTMENT}

This book is due on the last date stamped below, or on the date to which renewed.

Renewed books are subject to immediate recall.

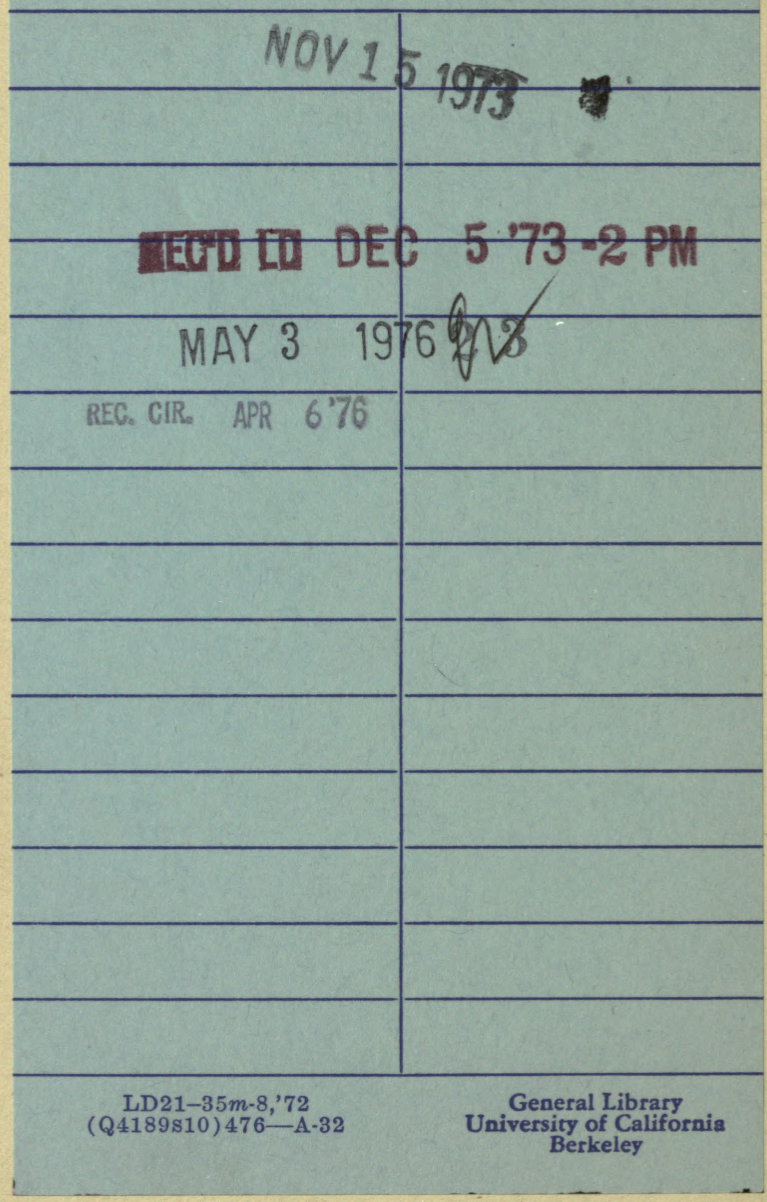


YC 20566 


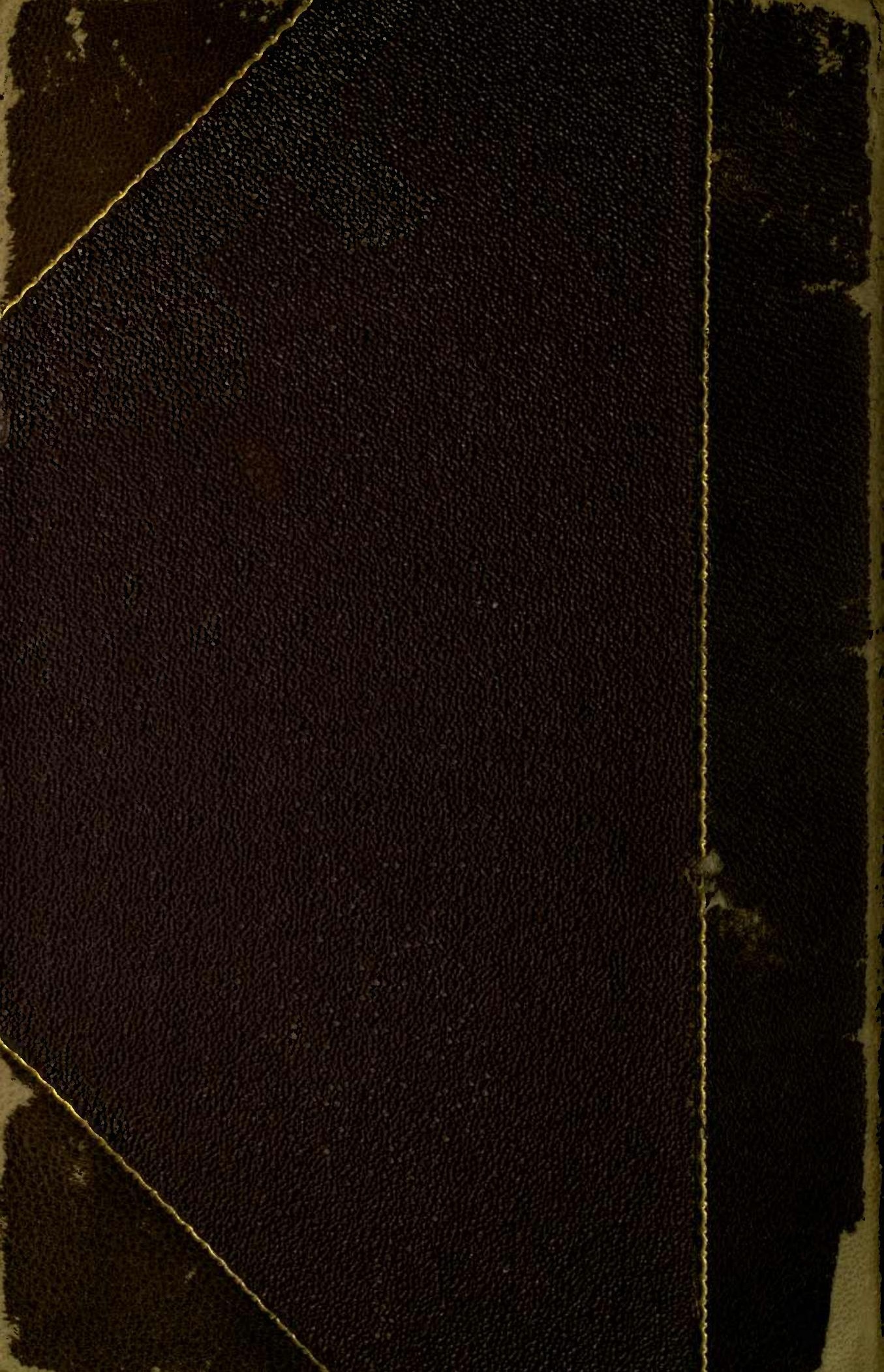

